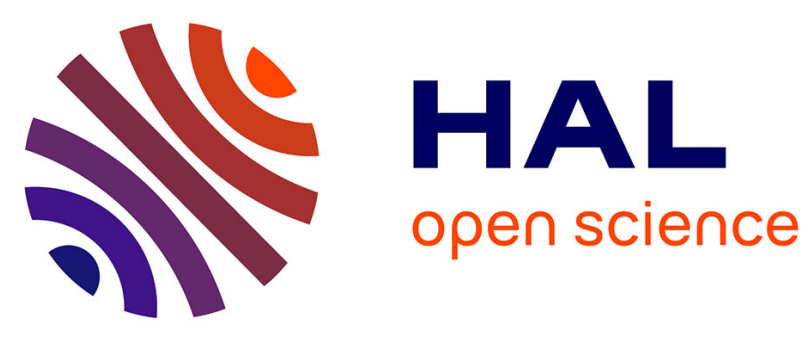

\title{
An Inertial-Aided Homography-Based Visual Servo Control Approach for (Almost) Fully Actuated Autonomous Underwater Vehicles
}

Szymon Krupínski, Guillaume Allibert, Minh-Duc Hua, Tarek Hamel

\section{- To cite this version:}

Szymon Krupínski, Guillaume Allibert, Minh-Duc Hua, Tarek Hamel. An Inertial-Aided HomographyBased Visual Servo Control Approach for (Almost) Fully Actuated Autonomous Underwater Vehicles. IEEE Transactions on Robotics, 2017, 33 (5), pp.1041-1060. 10.1109/TRO.2017.2700010 . hal01544726

\author{
HAL Id: hal-01544726 \\ https://hal.science/hal-01544726
}

Submitted on 22 Jun 2017

HAL is a multi-disciplinary open access archive for the deposit and dissemination of scientific research documents, whether they are published or not. The documents may come from teaching and research institutions in France or abroad, or from public or private research centers.
L'archive ouverte pluridisciplinaire HAL, est destinée au dépôt et à la diffusion de documents scientifiques de niveau recherche, publiés ou non, émanant des établissements d'enseignement et de recherche français ou étrangers, des laboratoires publics ou privés. 


\title{
An inertial-aided homography-based visual servo control approach for (almost) fully-actuated Autonomous Underwater Vehicles
}

\author{
Szymon Krupínski, Guillaume Allibert, Minh-Duc Hua, and Tarek Hamel
}

\begin{abstract}
A nonlinear inertial-aided image-based visual servo control approach for the stabilisation of (almost) fully-actuated autonomous underwater vehicles (AUVs) is proposed. It makes use of the homography matrix between two images of a planar scene as feedback information while the system dynamics are exploited in a cascade manner in control design: an outer-loop control defines a reference setpoint based on the homography matrix and an inner-loop control ensures the stabilisation of the setpoint by assigning the thrust and torque controls. Unlike conventional solutions that only consider the system kinematics, the proposed control scheme is novel in considering the full system dynamics (incorporating all degrees of freedom, nonlinearities and couplings as well as interactions with the surrounding fluid) and in not requiring information of the relative depth and normal vector of the observed scene. Augmented with integral corrections, the proposed controller is robust with respect to model uncertainties and disturbances. The almost global asymptotic stability of the closed-loop system is demonstrated, which is the largest domain of attraction one can achieve by means of continuous feedback control. Simulation results illustrating these properties on a realistic AUV model subjected to a sea current are presented and finally experimental results on a real AUV are reported.
\end{abstract}

Index Terms-AUV, nonlinear control, visual servoing, homography-based control, Lyapunov analysis

\section{INTRODUCTION}

Unlike aerial robotics that has witnessed an impressive growth during the last decade, little progress can be reported in AUV research and development. AUV applications have been drastically limited by the lack of global positioning system, particularly due to the attenuation of electromagnetic waves in water that prevents the implementation of GPS-like global positioning and wireless communication at high data rate. Typically, AUVs carry inertial sensors to estimate the vehicle's orientation, often improved by exploiting measurements of both a magnetic compass and a Doppler Velocity Log (DVL). They are also often equipped with sonars (scanning, multibeam or side-scan) or optical systems to provide information about the surrounding environment, leading to an obvious interest in the development of sensor-based navigation systems for applications in close proximity of the ocean floor and amidst obstacles (e.g., structure inspection, pipe/cable inspection, autonomous manipulation, mine hunting).

S. Krupínski is with Cybernetix, a Technip Company, Marseille, France. Email: szymon.krupinski@cybernetix.fr.

G. Allibert (corresponding author), M.-D. Hua, and T. Hamel are with Université of Côte d'Azur, CNRS, I3S, France. Emails: allibert(thamel,hua)@i3s.unice.fr.

This work was supported by the CNRS-PEPS project "CONGRE" and by Cybernetix company (Technip group).
The dynamics of AUVs are highly nonlinear and the translational and rotational dynamics are highly coupled, essentially due to added mass effects [10], [24]. The vehicles are often subjected to strong perturbations due to sea currents. Therefore, robust nonlinear control design for AUVs is particularly important. There is a considerable body of works on control design for AUVs. Different control approaches ranging from linear PID [2], LQR [30], $H_{\infty}$ [11], optimal control [40] to nonlinear control techniques such as sliding mode control [18], [23], Lyapunov backstepping-based control [1], [4], [35], and Lyapunov model-based control [34], [39] have been developed. However, they mostly concern the tracking problem of preprogrammed trajectories with little regard to the local topography of the environment. Autonomous navigation of AUVs in an unknown or partially known environment and in proximity to obstacles is the central problem due to the nature of missions such as pipe/cable inspection [3], [20], [38], mine hunting [33], or autonomous manipulation [27], etc.

This paper addresses specifically the inspection task of an AUV for potential industrial applications. Our preferred approach is based on high-bandwidth sensor-based control that reacts appropriately to the vehicle's environment and allows for behaviors such as the precise stabilisation with respect to (w.r.t.) a specific target. Although several types of sensors can be used, the video camera remains an excellent candidate. By using camera(s) as the primary sensor for relative position, the control problem can be cast into either Position-Based Visual Servo (PBVS) or Image-Based Visual Servo (IBVS) control problems [8], [9]. Classical visual servo control techniques have been developed for serial-link robotic manipulators and then for mobile ground vehicles (well summarized in [8], [9]), and more recently for aerial robotic vehicles [13], [14], [28], [32]. In underwater robotics, few attempts have been carried out using vision sensors to perform tasks related to man-made structures such as pipeline following using linear features [20], [38], station keeping using point features [25], or positioning using image mosaicking [12]. Both stereo and monocular visions have been exploited in the context of AUVs. In the case where the full pose (i.e. position and orientation) reconstruction is possible, existing position-based controllers can be applied [31], [37]. The case of monocular vision without the assumption of planarity of the target and knowledge of its geometry is more challenging because the pose cannot be fully reconstructed from visual data. However, as in the case of robotic manipulators and aerial robots [9], [28], [32], monocular vision can be sufficient to achieve sta- 
bilisation for (almost) fully-actuated AUVs in front of a planar target using $2 \frac{1}{2} \mathrm{D}$ servoing [7], [25], [41], essentially based on the work of Malis et al. [26]. Recently, an advanced IBVS control scheme has been proposed by Benhimane and Malis [5] using the homography matrix that encodes transformation information between two images of the same planar target and that can be directly retrieved from the corresponding images. This homography-based visual servoing (HBVS) scheme is a purely kinematic control, initially designed for fully-actuated manipulators. However, its stability and convergence are only established on the basis of local analysis and has never been studied when taking the system dynamics into account.

HBVS control can be applied to numerous AUV applications once a locally planar visual target is available. For instance, station keeping using a downward-looking camera to observe the ocean floor is a classical application. One can also mention stabilisation or positioning in front of a man-made subsea manifold for high-resolution imaging, monitoring, or inspection, or for manipulation like valve-turning, or for maintenance like cleaning, repairing, or changing underwater structures. Finally, docking on a planar docking station is also a relevant application for HBVS control.

The HBVS control problem has also been investigated for underactuated aerial vehicles [28], [32]. In [28], additional information such as the orientation measurement of the camera w.r.t. the target is assumed to be available. In contrast, the HBVS solution proposed in [32] only makes use of the homography matrix along with gyrometer measurements. However, only local stability is proved based on Lyapunov-like analysis. In fact, the consideration of the vehicle dynamics within control design is crucial to obtain provable (strong) stability. These works also show that significant efforts are required in order to eliminate the assumptions about the precise knowledge of the geometry of the planar target. The present study is in line with these efforts. Although the AUV system under consideration in this paper is (almost) fully-actuated, the strongly coupled translational and rotational dynamics represents another difficulty. In contrast with existing HBVS solutions, an important original outcome of the proposed HBVS control approach is related to the obtention of almost global asymptotic stability by means of continuous feedback control. This is the largest possible domain of attraction that one can achieve with continuous feedback control because the topological obstruction of a rotation group excludes the existence of continuous global stabilisers [6]. To our knowledge, our work is the first to address the HBVS problem of AUVs by taking the full nonlinear and highly coupled dynamics into account; and it is also the first to achieve almost global asymptotic stability.

This paper is organized as follows. Notation, system modeling are described in Section III In Section [III] we first state the HBVS problem for fully-actuated systems, and then discuss about the local nature of a state-of-the-art kinematic-based HBVS control proposed in [5]. In Section III-C] we propose a novel inertial-aided HBVS control approach for fully-actuated mechanical systems. Then, in Section IV an adaptation of this control approach to (almost) fully-actuated AUVs is derived by developing a novel inner-loop controller for the stabilisation of reference velocity setpoint. Simulation results on a realistic AUV model are presented in Section $\mathrm{V}$, illustrating the performance and robustness of the proposed approach and showing its superior performance w.r.t. the kinematic-based HBVS control [5]. In Section VI convincing experimental results on the AUV Girona 500 are reported. A video is provided as supplementary material. Finally, concluding remarks and future works are provided in Section VII A primary version of this work has been presented in [15] and a part of experimental results has been reported in [21].

\section{AUV SYSTEM MODELLING}

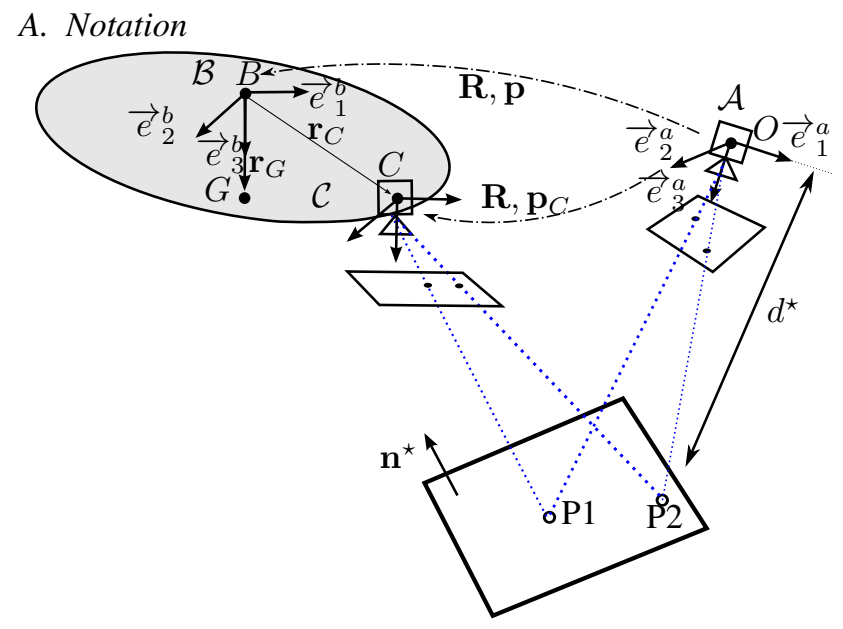

Fig. 1: Notation.

The considered AUV is modeled as a rigid body immersed in a fluid. The following notation is used (see Fig. 1).

- $G$ and $B$ are the vehicle's center of mass (CoM) and center of buoyancy (CoB), respectively, $m$ its mass and $\mathbf{J}_{0}$ its inertia matrix. Let $l$ denote the distance between $G$ and $B$.

- $\mathcal{A}=\left\{O ; \vec{e}_{1}^{a}, \vec{e}_{2}^{a}, \vec{e}_{3}^{a}\right\}$ is an inertial frame. $\mathcal{B}=$ $\left\{B ; \vec{e}_{1}^{b}, \vec{e}_{2}^{b}, \vec{e}_{3}^{b}\right\}$ is a frame attached to the AUV, with origin coinciding with the CoB. $\mathcal{C}=\left\{C ; \vec{e}_{1}^{c}, \vec{e}_{2}^{c}, \vec{e}_{3}^{c}\right\}$ is a frame attached to the camera, displaced from the origin of $\mathcal{B}$ by a vector $\overrightarrow{B C}$ and keeping its base vectors parallel to those of $\mathcal{B}$. Let $\mathbf{r}_{C} \in \mathbb{R}^{3}$ and $\mathbf{r}_{G} \in \mathbb{R}^{3}$ denote the vectors of coordinates expressed in the frame $\mathcal{B}$ of $\overrightarrow{B C}$ and $\overrightarrow{B G}$, respectively.

- The orientation (i.e. attitude) of $\mathcal{B}$ w.r.t. $\mathcal{A}$ is represented by the rotation matrix $\mathbf{R} \in \mathrm{SO}(3)$. The position vectors of the origins of $\mathcal{B}$ and $\mathcal{C}$, expressed in $\mathcal{A}$, are denoted as $\mathbf{p}$ and $\mathbf{p}_{C}$, respectively. Therefore, $\mathbf{p}=\mathbf{p}_{C}-\mathbf{R}_{C}$.

- The angular velocity vector of $\mathcal{B}$ relative to $\mathcal{A}$, expressed in $\mathcal{B}$, is denoted as $\boldsymbol{\Omega}=\left[\omega_{1}, \omega_{2}, \omega_{3}\right]^{\top} \in \mathbb{R}^{3}$. The translational (or linear) velocity vectors of the origins of $\mathcal{B}$ and $\mathcal{C}$, expressed in $\mathcal{B}$, are denoted as $\mathbf{V} \in \mathbb{R}^{3}$ and $\mathbf{V}_{C} \in \mathbb{R}^{3}$ respectively. We have $\mathbf{V}=\mathbf{V}_{C}-\boldsymbol{\Omega} \times \mathbf{r}_{C}$.

- $\mathbf{v}_{f}$ and $\mathbf{V}_{f}$ are the vector of coordinates of the current velocity in $\mathcal{A}$ and $\mathcal{B}$ respectively. In this work, $\mathbf{v}_{f}$ is assumed constant. $\mathbf{V}_{h} \triangleq \mathbf{V}-\mathbf{V}_{f}$ is the vector of coordinates of the CoB's velocity w.r.t. the fluid.

- $\mathbf{e}_{g} \in S^{2}$ (the unit 2-sphere) is the gravity direction expressed in the inertial frame $\mathcal{A}$. Let $g$ denote the gravity constant, i.e. $g \approx 9.81\left(\mathrm{~m} / \mathrm{s}^{2}\right)$.

- $\left\{\mathbf{e}_{1}, \mathbf{e}_{2}, \mathbf{e}_{3}\right\}$ denotes the canonical basis of $\mathbb{R}^{3} . \mathbf{I}_{3}$ is the identity matrix of $\mathbb{R}^{3 \times 3}$. For all $\mathbf{u} \in \mathbb{R}^{3}$, the notation 
$\mathbf{u}_{\times}$denotes the skew-symmetric matrix associated with the cross product by $\mathbf{u}$, i.e., $\mathbf{u}_{\times} \mathbf{v}=\mathbf{u} \times \mathbf{v}, \forall \mathbf{v} \in \mathbb{R}^{3}$. The operation $\operatorname{vex}($.$) is defined such that \operatorname{vex}\left(\mathbf{u}_{\times}\right)=\mathbf{u}, \forall \mathbf{u} \in \mathbb{R}^{3}$. The Euclidean norm in $\mathbb{R}^{n}$ is denoted as $|\cdot|$ and $(\cdot)^{\top}$ denotes the transpose operator. $\operatorname{sat}^{\delta}(\cdot) \in \mathbb{R}^{n}$, with $\delta>0$, is the classical saturation function defined by $\operatorname{sat}^{\delta}(\mathbf{x}) \triangleq$ $\mathbf{x} \min (1, \delta /|\mathbf{x}|), \forall \mathbf{x} \in \mathbb{R}^{n}$. The shorten notations $S(\cdot), C(\cdot)$, $T(\cdot)$, and $\cot (\cdot)$ are used for the sine, cosine, tangent, and cotangent operators.

\section{B. Recall on system modeling}

Define $\mathbf{W}_{h} \triangleq\left[\mathbf{V}_{h}^{\top}, \mathbf{\Omega}^{\top}\right]^{\top} \in \mathbb{R}^{6}$. When characterised at the $\mathrm{CoB}$, the kinetic energy of the vehicle is given by (see [24]) $E_{B}=\frac{1}{2} \mathbf{W}_{h}^{\top} \mathbf{M}_{B} \mathbf{W}_{h}, \quad$ with $\quad \mathbf{M}_{B} \triangleq\left[\begin{array}{cc}m \mathbf{I}_{3} & -m \mathbf{r}_{G \times} \\ m \mathbf{r}_{G \times} & \mathbf{J}_{0}\end{array}\right]$. According to Kirchhoff and Lamb theory [22], the kinetic energy of the liquid surrounding the vehicle is given by

$$
E_{F}=\frac{1}{2} \mathbf{W}_{h}^{\top} \mathbf{M}_{A} \mathbf{W}_{h}, \quad \text { with } \quad \mathbf{M}_{A} \triangleq\left[\begin{array}{ll}
\mathbf{M}_{A}^{11} & \mathbf{M}_{A}^{12} \\
\mathbf{M}_{A}^{21} & \mathbf{M}_{A}^{22}
\end{array}\right],
$$

with $\mathbf{M}_{A} \in \mathbb{R}^{6 \times 6}$ the added mass matrix, which is approximately constant and symmetric [10]. The total kinetic energy of the body-fluid system is $E_{T}=E_{B}+E_{F}=\frac{1}{2} \mathbf{W}_{h}^{\top} \mathbf{M}_{T} \mathbf{W}_{h}$, where $\mathbf{M}_{T}$ is given by

$$
\mathbf{M}_{T}=\mathbf{M}_{B}+\mathbf{M}_{A}=\left[\begin{array}{cc}
\mathbf{M} & \mathbf{D}^{\top} \\
\mathbf{D} & \mathbf{J}
\end{array}\right]
$$

with $\mathbf{M} \triangleq m \mathbf{I}_{3}+\mathbf{M}_{A}^{11}, \mathbf{J} \triangleq \mathbf{J}_{0}+\mathbf{M}_{A}^{22}, \mathbf{D} \triangleq m \mathbf{r}_{G \times}+\mathbf{M}_{A}^{21}$. The matrices $\mathbf{M}_{A}^{11}$ and $\mathbf{M}_{A}^{22}$ are often referred to as added mass and added inertia matrices, respectively. One derives the translational and rotational momentums as follows:

$$
\mathbf{P}_{h}=\frac{\partial E_{T}}{\partial \mathbf{V}_{h}}=\mathbf{M} \mathbf{V}_{h}+\mathbf{D}^{\top} \boldsymbol{\Omega}, \boldsymbol{\Pi}_{h}=\frac{\partial E_{T}}{\partial \boldsymbol{\Omega}}=\mathbf{J} \boldsymbol{\Omega}+\mathbf{D} \mathbf{V}_{h}
$$

Then, the equations of motion are given by [24]:

$$
\begin{aligned}
\dot{\mathbf{p}} & =\mathbf{R V} \\
\dot{\mathbf{R}} & =\mathbf{R} \boldsymbol{\Omega}_{\times} \\
\dot{\mathbf{P}}_{h} & =\mathbf{P}_{h} \times \boldsymbol{\Omega}+\mathbf{F}_{c}+\mathbf{F}_{G B}+\mathbf{F}_{d} \\
\dot{\boldsymbol{\Pi}}_{h} & =\boldsymbol{\Pi}_{h} \times \boldsymbol{\Omega}+\mathbf{P}_{h} \times \mathbf{V}_{h}+\boldsymbol{\Gamma}_{c}+m g \mathbf{r}_{G} \times \mathbf{R}^{\top} \mathbf{e}_{g}+\boldsymbol{\Gamma}_{d}
\end{aligned}
$$

where $\mathbf{F}_{c} \in \mathbb{R}^{3}$ and $\boldsymbol{\Gamma}_{c} \in \mathbb{R}^{3}$ are the force and torque control vector inputs at our disposal, $\mathbf{F}_{G B} \triangleq\left(m g-F_{B}\right) \mathbf{R}^{\top} \mathbf{e}_{g}$ is the sum of the gravitational and buoyancy forces, and the hydrodynamic damping force and torque vectors $\mathbf{F}_{d}$ and $\boldsymbol{\Gamma}_{d}$ are modeled as:

$$
\left\{\begin{array}{l}
\mathbf{F}_{d}=-\left(\mathbf{D}_{V l}+\left|\mathbf{V}_{h}\right| \mathbf{D}_{V q}\right) \mathbf{V}_{h} \\
\boldsymbol{\Gamma}_{d}=-\left(\mathbf{D}_{\Omega l}+|\boldsymbol{\Omega}| \mathbf{D}_{\Omega q}\right) \boldsymbol{\Omega}
\end{array}\right.
$$

with damping matrices $\mathbf{D}_{V l}, \mathbf{D}_{V q}, \mathbf{D}_{\Omega l}, \mathbf{D}_{\Omega q} \in \mathbb{R}^{3 \times 3}$.

\section{Model for control design}

Either the momentum terms $\mathbf{P}_{h}, \mathbf{\Pi}_{h}$ or their dynamics 3c(3d) involve unknown current velocity $\mathbf{V}_{f}$, which complicates the control design process. Therefore, we rewrite System (3) as follows:

$$
\begin{aligned}
\dot{\mathbf{p}} & =\mathbf{R V} \\
\dot{\mathbf{R}} & =\mathbf{R} \boldsymbol{\Omega}_{\times} \\
\dot{\mathbf{P}} & =\mathbf{P} \times \boldsymbol{\Omega}+\mathbf{F}_{c}+\mathbf{F}_{G B}+\overline{\mathbf{F}}_{d}+\boldsymbol{\Delta}_{F} \\
\dot{\boldsymbol{\Pi}} & =\mathbf{\Pi} \times \boldsymbol{\Omega}+\mathbf{P} \times \mathbf{V}+\boldsymbol{\Gamma}_{c}+m g \mathbf{r}_{G} \times \mathbf{R}^{\top} \mathbf{e}_{g}+\boldsymbol{\Gamma}_{d}+\boldsymbol{\Delta}_{\Gamma}
\end{aligned}
$$

with new momentum terms $\mathbf{P} \triangleq \mathbf{M V}+\mathbf{D}^{\top} \boldsymbol{\Omega}, \boldsymbol{\Pi} \triangleq \mathbf{J} \boldsymbol{\Omega}+$ $\mathbf{D V}$, and new dissipative force $\overline{\mathbf{F}}_{d} \triangleq-\left(\mathbf{D}_{V l}+|\mathbf{V}| \mathbf{D}_{V q}\right) \mathbf{V}$, and "disturbance" terms $\boldsymbol{\Delta}_{F}$ and $\boldsymbol{\Delta}_{\Gamma}$ given by:

$$
\begin{aligned}
\boldsymbol{\Delta}_{F} \triangleq & -\mathbf{M} \boldsymbol{\Omega}_{\times} \mathbf{V}_{f}-(\mathbf{M V})_{f} \boldsymbol{\Omega}+\mathbf{F}_{d}-\overline{\mathbf{F}}_{d} \\
\boldsymbol{\Delta}_{\Gamma} \triangleq & \left(\mathbf{M} \mathbf{V}_{f}\right)_{\times} \mathbf{V}_{f}-(\mathbf{M V})_{\times} \mathbf{V}-(\mathbf{M V})_{\times} \mathbf{V}_{f} \\
& -\mathbf{D} \boldsymbol{\Omega}_{\times} \mathbf{V}_{f}-(\mathbf{D V})_{\times} \boldsymbol{\Omega} .
\end{aligned}
$$

The disturbance terms $\boldsymbol{\Delta}_{F}$ and $\boldsymbol{\Delta}_{\Gamma}$ are null if the current velocity is null, i.e. $\mathbf{v}_{f}=\mathbf{0}$. Otherwise, they should be addressed using either an estimator (e.g. high-gain type) or integral compensation actions.

Since our control objective concerns fixed-point stabilisation, the disturbance terms $\boldsymbol{\Delta}_{F}$ and $\boldsymbol{\Delta}_{\Gamma}$ would eventually converge to constant values. Therefore, in the sequel the system's equations (5) will be used for control design, with $\boldsymbol{\Delta}_{F}$ and $\boldsymbol{\Delta}_{\Gamma}$ considered as unknown constant vectors.

\section{HOMOGRAPHY-BASED VISUAL SERVO (HBVS) CONTROL OF FULLY-ACTUATED MECHANICAL SYSTEMS}

\section{A. Problem formulation}

A reference image of a planar target is taken at some desired pose. Based on this reference image and the current image, the control objective consists in stabilising the camera's pose to the desired pose. The inertial frame $\mathcal{A}$ is chosen attaching to the reference pose of the camera (Fig. 1). Assume that the camera provides the measurement of the homography matrix $\mathbf{H}$, which is given by [5]:

$$
\mathbf{H}=\mathbf{R}^{\top}-\frac{1}{d^{\star}} \mathbf{R}^{\top} \mathbf{p}_{C} \mathbf{n}^{\star \top}
$$

where $d^{\star}$ is the distance between the target plane and the camera optical center (i.e. depth), and $\mathbf{n}^{\star}=\left[n_{1}^{\star}, n_{2}^{\star}, n_{3}^{\star}\right]^{\top}$ is the unit vector normal to the target plane expressed in the reference camera frame (see Fig. 11. It is verified that the dynamics of $\mathbf{H}$ are given by:

$$
\dot{\mathbf{H}}=-\boldsymbol{\Omega}_{\times} \mathbf{H}-\frac{1}{d^{\star}} \mathbf{V}_{C} \mathbf{n}^{\star \top} .
$$

Remark 1. In the case where the camera frame $\mathcal{C}_{t}$ (with the subscript " $t$ " used for "true") is not aligned with the bodyfixed frame $\mathcal{B}$ like in the second experiment reported in Section VI, it is still possible to derive the homography matrix $\mathbf{H}$ like it is estimated from images provided by a "virtual" downwardlooking camera (i.e. the "virtual" camera frame $\mathcal{C}$ aligned with the body-fixed frame $\mathcal{B})$. Indeed, denoting $\mathbf{R}_{C} \in \mathrm{SO}(3)$ as the rotation matrix of the "true" camera frame $\mathcal{C}_{t}$ relative to the "virtual" camera frame $\mathcal{C}$, and $\mathbf{H}_{t}$ as the homography matrix estimated from the "true" camera, then it is not difficult to verify that $\mathbf{H}=\mathbf{R}_{C} \mathbf{H}_{t} \mathbf{R}_{C}^{\top}$.

The control objective can be stated as the stabilisation of $\mathbf{H}$ about the identity matrix $\mathbf{I}_{3}$, or equivalently the stabilisation of $\left(\mathbf{R}, \mathbf{p}_{C}\right)$ about $\left(\mathbf{I}_{3}, \mathbf{0}\right)$. HBVS control design difficulties lie in the fact that the depth $d^{\star}$ and the normal vector $\mathbf{n}^{\star}$ involved in the expression (6) of $\mathbf{H}$ are unknown and that this matrix only contains a coupled information of rotation and translation. 


\section{B. Discussions on an existing kinematic HBVS control}

Let us recall and discuss about a state-of-the-art kinematic HBVS control proposed in [5]. The authors define the visual errors $\mathbf{e}_{p}, \mathbf{e}_{\Theta} \in \mathbb{R}^{3}$ as [5]:

$$
\mathbf{e}_{p} \triangleq\left(\mathbf{I}_{3}-\mathbf{H}\right) \mathbf{m}^{\star}, \mathbf{e}_{\Theta} \triangleq \operatorname{vex}\left(\mathbf{H}^{\top}-\mathbf{H}\right)
$$

with some arbitrary unit vector $\mathbf{m}^{\star} \in S^{2}$ satisfying the following assumption.

Assumption 1. (See [5]) Assume that a unit vector $\mathbf{m}^{\star} \in S^{2}$ can be chosen such that $\mathbf{n}^{\star \top} \mathbf{m}^{\star}>0$.

Remark 2. Assumption 1 is not restrictive in practice. Indeed, in order for the visual target be viewed by the camera at the reference pose, the angle between the normal vector of the target plane and the opposite direction of the reference camera's axis must be contained in $[0, \pi / 2)$. For instance, if the camera points downwards then it is obvious that $\mathbf{n}^{\star \top}\left(-\mathbf{e}_{3}\right)>0$, suggesting one to choose $\mathbf{m}^{\star}=-\mathbf{e}_{3}$ without any prior knowledge of $\mathbf{n}^{\star}$.

Lemma 1. (See [5]) Under Assumption [1] the kinematic control law:

$$
\mathbf{V}_{C}=-k_{p} \mathbf{e}_{p}, \quad \mathbf{\Omega}=-k_{\Theta} \mathbf{e}_{\Theta},
$$

with $k_{p}, k_{\Theta}$ some positive gains, ensures the local exponential stability (LES) of the equilibrium $\left(\mathbf{R}, \mathbf{p}_{C}\right)=\left(\mathbf{I}_{3}, \mathbf{0}\right)$, or equivalently of $\mathbf{H}=\mathbf{I}_{3}$. Moreover, the function $\mathbf{e} \triangleq\left[\mathbf{e}_{p}^{\top}, \mathbf{e}_{\Theta}^{\top}\right]^{\top}$ is isomorphic to $\mathbf{H}$, i.e. $\mathbf{e}=0$ iif $\mathbf{H}=\mathbf{I}_{3}$.

The proof of Lemma 1 given in [5] is based on the linearised closed-loop system, taking $\mathbf{V}_{C}$ and $\boldsymbol{\Omega}$ as control inputs. However, for mechanical systems, instead of the linear and angular velocities, forces and torques should be used as control inputs for control design. For instance, only for discussions, let us consider the following simplified dynamical system:

$$
\left\{\begin{array}{l}
\dot{\mathbf{V}}_{C}=\mathbf{F}_{c} \\
\dot{\Omega}=\Gamma_{c}
\end{array}\right.
$$

with $\mathbf{F}_{c} \in \mathbb{R}^{3}$ and $\boldsymbol{\Gamma}_{c} \in \mathbb{R}^{3}$ control inputs. For this system, it is easy to stabilise $\mathbf{V}_{C}$ and $\boldsymbol{\Omega}$ at any smooth reference values $\mathbf{V}_{C r}$ and $\boldsymbol{\Omega}_{r}$, provided that the derivatives $\dot{\mathbf{V}}_{C r}$ and $\dot{\boldsymbol{\Omega}}_{r}$ (i.e. feed-forward terms) are computable by the controller. Indeed, applying the following $\mathrm{P}$ (proportional)-controller:

$$
\left\{\begin{array}{l}
\mathbf{F}_{c}=-k_{V}\left(\mathbf{V}_{C}-\mathbf{V}_{C r}\right)+\dot{\mathbf{V}}_{C r} \\
\boldsymbol{\Gamma}_{c}=-k_{\Omega}\left(\boldsymbol{\Omega}-\boldsymbol{\Omega}_{r}\right)+\dot{\boldsymbol{\Omega}}_{r}
\end{array}\right.
$$

with $k_{V}, k_{\Omega}$ positive gains, it is straightforward to verify that the equilibrium $\left(\mathbf{V}_{C}, \boldsymbol{\Omega}\right)=\left(\mathbf{V}_{C r}, \boldsymbol{\Omega}_{r}\right)$ of the controlled system is globally exponentially stable.

Therefore, in view of Lemma 1 one may attempt to define the reference velocities as $\mathbf{V}_{C r}=-k_{p} \mathbf{e}_{p}$ and $\boldsymbol{\Omega}_{r}=-k_{\Theta} \mathbf{e}_{\Theta}$ and apply controller (11) to System (7)+(10) in order to ensure the LES of the equilibrium $\left(\mathbf{H}, \mathbf{V}_{C}, \boldsymbol{\Omega}\right)=\left(\mathbf{I}_{3}, \mathbf{0}, \mathbf{0}\right)$. However, since the derivative of $\mathbf{e}_{p}$ and $\mathbf{e}_{\Theta}$ are not computable by the controller due to the unknown quantities $\mathbf{n}^{\star}$ and $d^{\star}$, it is impossible to compute the feed-forward terms $\dot{\mathbf{V}}_{C r}$ and $\dot{\boldsymbol{\Omega}}_{r}$ involved in (11). A popular and practical solution to this issue consists in neglecting the uncomputable feed-forward terms $\dot{\mathbf{V}}_{C r}$ and $\dot{\boldsymbol{\Omega}}_{r}$ in (11), i.e. setting $\dot{\mathbf{V}}_{C r}=\dot{\boldsymbol{\Omega}}_{r}=\mathbf{0}$. Curiously, no stability analysis for such a "hierarchical" PD(proportionalderivative)-controller can be found in literature. We state next its local stability property.
Lemma 2. Consider System (7) $+(10)$ and apply the "hierarchical" PD-controller:

$$
\mathbf{F}_{c}=-k_{V}\left(\mathbf{V}_{C}-\mathbf{V}_{C r}\right), \quad \boldsymbol{\Gamma}_{c}=-k_{\Omega}\left(\boldsymbol{\Omega}-\boldsymbol{\Omega}_{r}\right),
$$

with

$$
\mathbf{V}_{C r} \triangleq-k_{p} \mathbf{e}_{p}, \quad \mathbf{\Omega}_{r} \triangleq-k_{\Theta} \mathbf{e}_{\Theta} .
$$

and positive gains $k_{p}, k_{\Theta}, k_{V}, k_{\Omega}$. Then, the equilibrium $\left(\mathbf{H}, \mathbf{V}_{C}, \boldsymbol{\Omega}\right)=\left(\mathbf{I}_{3}, \mathbf{0}, \mathbf{0}\right)$ of the controlled system is locally exponentially stable.

The proof of Lemma 2 is given in Appendix A

Remark 3. In addition to the estimated homography, implementing the PD-controller $(12)+(13)$ requires the measurements of the linear and angular velocities which can be obtained from a DVL and an IMU, respectively.

Since mechanical systems are often subjected to unknown perturbations (e.g. induced by sea currents) and model parameter uncertainties, it is important in practice to add integral correction actions into the control law. This may suggest one to replace the "hierarchical" PD-controller (12) by the following "hierarchical" PID(proportional-integralderivative)-controller:

$$
\left\{\begin{array}{l}
\mathbf{F}_{c}=-k_{V}\left(\mathbf{V}_{C}-\mathbf{V}_{C r}\right)-k_{i V} \int_{0}^{t}\left(\mathbf{V}_{C}(s)-\mathbf{V}_{C r}(s)\right) d s \\
\boldsymbol{\Gamma}_{c}=-k_{\Omega}\left(\boldsymbol{\Omega}-\boldsymbol{\Omega}_{r}\right)-k_{i \Omega} \int_{0}^{t}\left(\boldsymbol{\Omega}(s)-\boldsymbol{\Omega}_{r}(s)\right) d s
\end{array}\right.
$$

with $k_{V}, k_{\Omega}, k_{i V}, k_{i \Omega}$ positive gains and $\mathbf{V}_{C r}, \boldsymbol{\Omega}_{r}$ defined by (13). However, integral correction actions may destabilise the controlled system, as a result of the following lemma (the proof is given in Appendix B.

Lemma 3. Apply the "hierarchical" PID-controller (14) to System $(7)+10)$. Then, the equilibrium $\left(\mathbf{H}, \mathbf{V}_{C}, \mathbf{\Omega}\right)=$ $\left(\mathbf{I}_{3}, \mathbf{0}, \mathbf{0}\right)$ of the controlled system is unstable if

$$
k_{i V}>k_{V}^{2} \quad \text { and } \quad a^{\star}>\frac{k_{V} k_{i V}}{k_{p}\left(k_{i V}-k_{V}^{2}\right)},
$$

with $a^{\star} \triangleq \frac{\left(\mathbf{n}^{\star \top} \mathbf{m}^{\star}\right)}{d^{\star}}$.

Remark 4. The main limitation of the kinematic controller (9), the "hierarchical" PD-controller (12) and the "hierarchical" PID-controller (14) is the local nature of the control design and analysis. The domain of attraction is, thus, difficult to be characterised. It can also be very limited when applying to strongly nonlinear systems such as AUVs, where their translational and rotational dynamics are strongly coupled and nonlinear due to added-mass effects. Simulation section $\nabla$ will provide more illustrations about this issue. Another limitation of both controllers (12) and (14) is that stability analysis can no longer be done in a "classical" and "practical" cascade manner (i.e. inner-outer loop control architecture).

In the sequel, we propose a novel HBVS control approach relying on the classical inner-outer loop strategy in the sense that the inner loop stabilises the linear and angular velocities $\mathbf{V}_{C}, \boldsymbol{\Omega}$ to any smooth reference values $\mathbf{V}_{C r}, \boldsymbol{\Omega}_{r}$, provided that their derivatives $\dot{\mathbf{V}}_{C r}, \dot{\boldsymbol{\Omega}}_{r}$ are available to control computations, while the outer loop makes use of $\mathbf{V}_{C r}, \boldsymbol{\Omega}_{r}$ as intermediate control variables to carry out the control objective (i.e. stabilise $\mathbf{H}$ about $\mathbf{I}_{3}$ or, equivalently, stabilise $\left(\mathbf{e}_{p}, \mathbf{e}_{\Theta}\right)$ about zero). 


\section{Proposed HBVS control design for fully-actuated systems}

As highlighted in Section III-B, for a fully-actuated system with force and torque control inputs, it is not difficult to design an inner-loop controller that ensures the convergence of the velocities $\left(\mathbf{V}_{C}, \boldsymbol{\Omega}\right)$ to any smooth reference velocities $\left(\mathbf{V}_{C r}, \boldsymbol{\Omega}_{r}\right)$, provided that $\dot{\mathbf{V}}_{C r}$ and $\dot{\boldsymbol{\Omega}}_{r}$ are computable. Assuming that such a controller is available, we now focus on the control design of the outer-loop level.

1) Reference linear velocity design: Using (7) and (8), one verifies that

$$
\dot{\mathbf{e}}_{p}=-\boldsymbol{\Omega} \times\left(\mathbf{e}_{p}-\mathbf{m}^{\star}\right)+a^{\star} \mathbf{V}_{C},
$$

with $a^{\star}$ defined in Lemma 3 . For control design insights, let us, for instance, proceed kinematic control design using the camera velocity $\mathbf{V}_{C}$ as control input, with the objective of stabilising $\mathbf{e}_{p}$ about zero globally. In view of (16), the control difficulty lies in the term $\boldsymbol{\Omega} \times \mathbf{m}^{\star}$ and also the unknown multiplicative constant $a^{\star}$, which is positive in view of Assumption 1

Lemma 4. (Kinematic control) Introduce the adaptive scalar dynamics:

$$
\dot{z}_{p}=\mathbf{e}_{p}^{\top}\left(\boldsymbol{\Omega} \times \mathbf{m}^{\star}\right), \quad z_{p}(0) \in \mathbb{R},
$$

and apply the kinematic control law

$$
\mathbf{V}_{C}=-k_{p} \mathbf{e}_{p}-z_{p} \boldsymbol{\Omega} \times \mathbf{m}^{\star},
$$

with $k_{p}>0$. Assume that $\Omega$ remains bounded. Then, the visual error $\mathbf{e}_{p}$ is globally asymptotically stabilised about zero.

The proof is reported in Appendix C

Remark 5. A difference between our kinematic control $\mathbf{V}_{C}$ (18) and the kinematic control (9) is that the global convergence of $\mathbf{e}_{p}$ to zero is obtained using only $\mathbf{V}_{C}$ as control input (i.e. without using $\Omega$ ). The other kinematic control variable $\Omega$ can, thus, be independently designed for the convergence of $\mathbf{e}_{\Theta}$ to zero.

In view of Lemma (4), one may define the reference linear velocity for the inner-loop control level as $\mathbf{V}_{C r}=-k_{p} \mathbf{e}_{p}-$ $z_{p} \boldsymbol{\Omega}_{r} \times \mathbf{m}^{\star}$. However, similarly to the problem discussed in Section III-B the derivative of $\mathbf{V}_{C r}$ is not available to the computation of the inner-loop control. To overcome this issue, the following modification to Lemma 4 is proposed next.

Proposition 1. Let $k_{p 1}, k_{p 2}, k_{z}, \Delta, \nabla$ denote some positive numbers. Introduce the following augmented system

$$
\left\{\begin{array}{l}
\dot{z}_{p}=\operatorname{sat}^{\nabla}\left(\mathbf{e}_{p}\right)^{\top}\left(\boldsymbol{\Omega}_{r} \times \mathbf{m}^{\star}\right)-k_{z} \nabla\left(z_{p}-\operatorname{sat}^{\Delta}\left(z_{p}\right)\right) \\
\dot{\hat{\mathbf{e}}}_{p}=-\boldsymbol{\Omega} \times \hat{\mathbf{e}}_{p}-k_{p 2}\left(\hat{\mathbf{e}}_{p}-\mathbf{e}_{p}\right)
\end{array}\right.
$$

with $z_{p}(0) \in \mathbb{R}$ and $\hat{\mathbf{e}}_{p}(0) \in \mathbb{R}^{3}$, and with $\Delta$ large enough such that $\Delta \geq 1 / a^{\star}$. Define the following reference linear velocity used by the inner-loop controller:

$$
\mathbf{V}_{C r} \triangleq-k_{p 1} \hat{\mathbf{e}}_{p}-z_{p} \boldsymbol{\Omega}_{r} \times \mathbf{m}^{\star}
$$

Assume that Assumption 1 is satisfied and that the reference angular velocity $\boldsymbol{\Omega}_{r}$ and its derivative are bounded and computable by the inner-loop controller. Apply any innerloop controller that ensures the global asymptotical stability and local exponential stability of the equilibrium $\left(\mathbf{V}_{C}, \boldsymbol{\Omega}\right)=$ $\left(\mathbf{V}_{C r}, \boldsymbol{\Omega}_{r}\right)$. There exist some positive numbers $\bar{\nabla}$ and $\bar{\kappa}_{p}$ such that if $\nabla>\bar{\nabla}$ and $\frac{k_{p 2}}{k_{p 1}}>\bar{\kappa}_{p}$ then $\mathbf{e}_{p}$ is globally stabilised about zero.

The proof is reported in Appendix D
2) Reference angular velocity design: In view of (20), the definition of the reference linear velocity $\mathbf{V}_{C r}$ depends on the reference angular velocity $\boldsymbol{\Omega}_{r}$. In the following, $\boldsymbol{\Omega}_{r}$ and its derivative $\dot{\boldsymbol{\Omega}}_{r}$ will be defined so as to ensure the convergence of $\mathbf{e}_{\Theta}$ to zero.

Proposition 2. Assume that all stability results of Proposition 11 hold. Define the angular velocity (used by the inner-loop control) as the solution to the following system:

$$
\dot{\boldsymbol{\Omega}}_{r}=-k_{\Theta 2} \boldsymbol{\Omega}_{r}-k_{\Theta 1} \mathrm{sat}^{\Delta_{\omega}}\left(\mathbf{e}_{\Theta}\right), \quad \boldsymbol{\Omega}_{r}(0) \in \mathbb{R}^{3},
$$

with positive numbers $k_{\Theta 1}, k_{\Theta 2}$ and $\Delta_{\omega}$. Then, the following properties hold:

1) $\boldsymbol{\Omega}_{r}$ and $\dot{\boldsymbol{\Omega}}_{r}$ remains bounded by $\frac{k_{\Theta 1} \Delta_{\omega}}{k_{\Theta 2}}$ and $2 k_{\Theta 1} \Delta_{\omega}$, respectively.

2) The equilibrium $\left(\mathbf{H}, \mathbf{V}_{C}, \boldsymbol{\Omega}\right)=\left(\mathbf{I}_{3}, \mathbf{0}, \mathbf{0}\right)$ of the controlled system is locally asymptotically stable (LAS). Furthermore, there exists a positive number $\underline{k}_{\Theta 1}$ such that if $k_{\Theta 1} \leq \underline{k}_{\Theta 1}$ then the equilibrium $\left(\mathbf{H}, \mathbf{V}_{C}, \boldsymbol{\Omega}\right)=$ $\left(\mathbf{I}_{3}, \mathbf{0}, \mathbf{0}\right)$ is locally exponentially stable (LES).

The proof is given in Appendix E Although the visual error variable $\mathbf{e}_{p}$ is globally stabilised about zero (Proposition 1, the convergence of the visual error variable $\mathbf{e}_{\Theta}$ to zero is only local (Proposition 2). In the sequel, we will show that stronger stability results can be obtained if an additional vectorial measurement is available to control design. In practice, vectorial measurements can be obtained using basic sensors such as accelerometer (to measure the gravity direction) or magnetometer (to measure the Earth's magnetic field), etc.

Assumption 2. Assume that a vectorial measurement in the body-fixed frame $\mathcal{B}$ of a known inertial unit vector $\mathbf{u} \in S^{2}$ is available for control computations, i.e. $\mathbf{R}^{\top} \mathbf{u}$ is known.

Under Assumption 2, our idea is to define the reference angular velocity vector (used by the inner-loop controller) as follows (instead of the solution to (21)):

$$
\boldsymbol{\Omega}_{r} \triangleq k_{u} \mathbf{u} \times \mathbf{R}^{\top} \mathbf{u}+\omega_{r} \mathbf{u}
$$

with $k_{u}>0$ and $\omega_{r} \in \mathbb{R}$ to be specified hereafter. We also rely on the inner-loop controller that must ensure not only the convergence of $\left(\mathbf{V}_{C}, \boldsymbol{\Omega}\right)$ to $\left(\mathbf{V}_{C r}, \boldsymbol{\Omega}_{r}\right)$ but also the convergence of $\mathbf{R}^{\top} \mathbf{u}$ to $\mathbf{u}$.

For any $\mathbf{u} \in S^{2}$, there exists a well-defined rotation matrix $\mathbf{R}_{u} \in \mathrm{SO}(3)$ such that $\mathbf{R}_{u} \mathbf{u}=\mathbf{e}_{3}$. For instance, if $\mathbf{u} \neq-\mathbf{e}_{3}$, such a rotation matrix is given by (Rodrigues' formula)

$$
\mathbf{R}_{u} \triangleq \mathbf{I}_{3}+\left(\mathbf{u} \times \mathbf{e}_{3}\right)_{\times}+\frac{\left(\mathbf{u} \times \mathbf{e}_{3}\right)_{\times}^{2}}{1+\mathbf{u}^{\top} \mathbf{e}_{3}} .
$$

Theorem 1. Assume that Assumption 1 is satisfied with $\mathbf{m}^{\star}=$ $\mathbf{R}_{u}^{\top} \overline{\mathbf{m}}^{\star} \in S^{2}$ and $\overline{\mathbf{m}}^{\star} \in \operatorname{span}\left(\mathbf{e}_{1}, \mathbf{e}_{3}\right)$. Define the reference angular velocity $\boldsymbol{\Omega}_{\mathbf{r}}$ by (22), where $\omega_{r}$ is the solution to the following system:

$$
\dot{\omega}_{r}=-k_{\Theta 2} \omega_{r}-k_{\Theta 1} \operatorname{sat}^{\Delta_{\omega}}\left(\bar{h}_{1,2}\right), \quad \omega_{r}(0) \in \mathbb{R},
$$

with positive numbers $k_{\Theta 1}, k_{\Theta 2}$ and $\Delta_{\omega}$, and $\bar{h}_{1,2}$ denoting the element at the intersection of the first row and second column of $\overline{\mathbf{H}} \triangleq \mathbf{R}_{u} \mathbf{H} \mathbf{R}_{u}^{\top}$. Define the reference translational velocity $\mathbf{V}_{C r}$ as in Proposition 1 Apply any innerloop controller that ensures the almost global asymptotical 
stability and local exponential stability of the equilibrium $\left(\mathbf{V}_{C}, \boldsymbol{\Omega}, \mathbf{R}^{\top} \mathbf{u}\right)=\left(\mathbf{V}_{C r}, \boldsymbol{\Omega}_{r}, \mathbf{u}\right)$. Then, there exist some positive numbers $\bar{\kappa}_{\Theta}$ and $\bar{\Delta}_{\omega}$ such that for all $k_{\Theta 1}, k_{\Theta 2}$ and $\Delta_{\omega}$ satisfying $k_{\Theta 2} / \sqrt{k_{\Theta 1}}>\bar{\kappa}_{\Theta}$ and $\Delta_{\omega}>\bar{\Delta}_{\omega}$, the homography matrix $\mathbf{H}$ is stabilised about $\mathbf{I}_{3}$ for almost all initial conditions.

The proof is given in Appendix $\mathrm{F}$.

Remark 6. Theorem 1 suggests one to choose a large value of $\Delta_{\omega}$ and a gain $k_{\Theta 2}$ much larger than $k_{\Theta 1}$. On the other hand, Proposition 1 suggests one to choose large values of $\Delta$ and $\nabla$ and a gain $k_{p 2}$ much larger than $k_{p 1}$.

Remark 7. Since the inner-loop controller ensures the convergence of $\mathbf{R}^{\top} \mathbf{u}$ to $\mathbf{u}$, the reference velocity $\boldsymbol{\Omega}_{r}$ given by (22) converges to $\omega_{r} \mathbf{u}$. Interestingly, if for some reason the vector $\mathbf{m}^{\star}$ is chosen parallel to $\mathbf{u}$ (for example in the case where $\mathbf{u}=\mathbf{e}_{3}$ (gravity direction) and $\mathbf{m}^{\star}=-\mathbf{e}_{3}$ (when using a downward-looking camera)), then the outer-loop controller (19)-20) given in Proposition (1) can be simplified to

$$
\mathbf{V}_{C r}=-k_{p 1} \hat{\mathbf{e}}_{p} \text {, }
$$

with $k_{p 1}>0$ and $\hat{\mathbf{e}}_{p}$ the solution to the following equation:

$$
\dot{\hat{\mathbf{e}}}_{p}=-\omega_{r} \mathbf{u} \times \hat{\mathbf{e}}_{p}-k_{p 2}\left(\hat{\mathbf{e}}_{p}-\mathbf{e}_{p}\right) \text {. }
$$

The adaptive term $z_{p}$ given in (19) is no longer involved in the outer-loop controller $\mathbf{V}_{C r}$, and the simpler proof (left to the interested reader) of global convergence of $\mathbf{e}_{p}$ to zero no longer requires any condition on $k_{p 1}$ and $k_{p 2}$.

\section{APPLICATION TO INERTIAL-AIDED HBVS CONTROL OF (ALMOST) FULLY-ACTUATED AUVS}

For AUV navigation, IMU is a very basic sensor. In addition to the angular velocity $\Omega$, it also provides an approximate estimate/measurement of the gravity direction in the body frame $\mathcal{B}$ (i.e. $\mathbf{R}^{\top} \mathbf{e}_{g}$ ) (under the assumption of weak linear accelerations of the vehicle). Note that the gravity direction can also be estimated without relying on the assumption of weak accelerations by fusing IMU measurements with DVL measurements [17]. Using this vectorial estimate, we rewrite the expression 22) of the reference angular velocity $\boldsymbol{\Omega}_{r}$ as

$$
\boldsymbol{\Omega}_{r}=k_{u} \mathbf{e}_{g} \times \mathbf{R}^{\top} \mathbf{e}_{g}+\omega_{r} \mathbf{e}_{g} .
$$

with $k_{u}>0$ and $\omega_{r}$ specified by the outer-loop control level as in Theorem 1 .

Since the outer-loop control design has been addressed in the previous section, in view of Theorem 1 it only now matters to design an inner-loop controller that ensures the convergence of $\left(\mathbf{V}, \boldsymbol{\Omega}, \mathbf{R}^{\top} \mathbf{e}_{g}\right)$ to $\left(\mathbf{V}_{r}, \boldsymbol{\Omega}_{r}, \mathbf{e}_{g}\right)$, with $\mathbf{V}_{r} \triangleq \mathbf{V}_{C r}-\boldsymbol{\Omega}_{r} \times \mathbf{r}_{C}$.

Define the velocity error variables

$$
\widetilde{\mathbf{V}} \triangleq \mathbf{V}-\mathbf{V}_{r}, \quad \widetilde{\boldsymbol{\Omega}} \triangleq \boldsymbol{\Omega}-\boldsymbol{\Omega}_{r} .
$$

Then, using $5 \mathrm{c}$, , 5d], 26), one obtains the following coupled error dynamics:

$$
\begin{aligned}
\mathbf{M} \dot{\tilde{\mathbf{V}}}+\mathbf{D}^{\top} \dot{\tilde{\mathbf{\Omega}}}= & \left(\mathbf{M V}+\mathbf{D}^{\top} \boldsymbol{\Omega}\right)_{\times} \widetilde{\boldsymbol{\Omega}}+\left(\mathbf{M} \widetilde{\mathbf{V}}+\mathbf{D}^{\top} \widetilde{\boldsymbol{\Omega}}\right)_{\times} \boldsymbol{\Omega}_{r} \\
& +\mathbf{F}_{G B}+\overline{\mathbf{F}}_{d}+\boldsymbol{\Delta}_{F}+\mathbf{F}_{r}+\mathbf{F}_{c} \\
\mathbf{J} \dot{\widetilde{\mathbf{\Omega}}}+\mathbf{D} \dot{\tilde{\mathbf{V}}=} & (\mathbf{J} \boldsymbol{\Omega}+\mathbf{D V})_{\times} \widetilde{\boldsymbol{\Omega}}+\left(\mathbf{M V}+\mathbf{D}^{\top} \boldsymbol{\Omega}\right)_{\times} \widetilde{\mathbf{V}} \\
& +(\mathbf{J} \widetilde{\boldsymbol{\Omega}}+\mathbf{D} \widetilde{\mathbf{V}})_{\times} \boldsymbol{\Omega}_{r}+\left(\mathbf{M} \widetilde{\mathbf{V}}+\mathbf{D}^{\top} \widetilde{\boldsymbol{\Omega}}\right)_{\times} \mathbf{V}_{r} \\
& +m g \mathbf{r}_{G} \times \mathbf{R}^{\top} \mathbf{e}_{g}+\boldsymbol{\Gamma}_{d}+\boldsymbol{\Delta}_{\Gamma}+\boldsymbol{\Gamma}_{r}+\boldsymbol{\Gamma}_{c}
\end{aligned}
$$

\footnotetext{
${ }^{1}$ Asymptotical stability for all initial conditions other than on a set of measure zero.
}

where the terms $\mathbf{F}_{r}$ and $\boldsymbol{\Gamma}_{r}$ are defined by

$\mathbf{F}_{r} \triangleq-\mathbf{M} \dot{\mathbf{V}}_{r}-\mathbf{D}^{\top} \dot{\boldsymbol{\Omega}}_{r}+\left(\mathbf{M} \mathbf{V}_{r}+\mathbf{D}^{\top} \boldsymbol{\Omega}_{r}\right)_{\times} \boldsymbol{\Omega}_{r}$

$\boldsymbol{\Gamma}_{r} \triangleq-\mathbf{J} \dot{\boldsymbol{\Omega}}_{r}-\mathbf{D} \dot{\mathbf{V}}_{r}+\left(\mathbf{J} \boldsymbol{\Omega}_{r}+\mathbf{D} \mathbf{V}_{r}\right)_{\times} \boldsymbol{\Omega}_{r}+\left(\mathbf{M} \mathbf{V}_{r}+\mathbf{D}^{\top} \boldsymbol{\Omega}_{r}\right)_{\times} \mathbf{V}_{r}$

From here, the inner-loop controller is proposed next, with proof given in Appendix $\mathrm{G}$

Proposition 3. Consider the system dynamics 27a-27b and apply the following controller:

$$
\left\{\begin{aligned}
\mathbf{F}_{c}= & -\mathbf{K}_{V} \widetilde{\mathbf{V}}-\mathbf{K}_{i V} \mathbf{z}_{V}-\left(\mathbf{M} \widetilde{\mathbf{V}}+\mathbf{D}^{\top} \widetilde{\boldsymbol{\Omega}}\right) \times \boldsymbol{\Omega}_{r} \\
& +\mathbf{M}\left(\widetilde{\boldsymbol{\Omega}} \times \mathbf{V}_{r}\right)+\mathbf{D}^{\top}\left(\widetilde{\boldsymbol{\Omega}} \times \boldsymbol{\Omega}_{r}\right)-\mathbf{F}_{r}-\mathbf{F}_{G B}-\overline{\mathbf{F}}_{d r} \\
\boldsymbol{\Gamma}_{c}= & -\mathbf{K}_{\Omega} \widetilde{\boldsymbol{\Omega}}-\mathbf{K}_{i \Omega} \mathbf{z}_{\Omega}-(\mathbf{J} \widetilde{\boldsymbol{\Omega}}) \times \boldsymbol{\Omega}_{r}-\left(\mathbf{D}^{\top} \widetilde{\boldsymbol{\Omega}}\right) \times \mathbf{V}_{r} \\
& -\boldsymbol{\Gamma}_{r}-m g \mathbf{r}_{G} \times \mathbf{R}^{\top} \mathbf{e}_{g}-\boldsymbol{\Gamma}_{d r}
\end{aligned}\right.
$$

with $\mathbf{K}_{V}, \mathbf{K}_{\Omega}, \mathbf{K}_{i V}, \mathbf{K}_{i \Omega}$ some positive diagonal $3 \times 3$ gain matrices, $\mathbf{z}_{V} \triangleq \int_{0}^{t} \widetilde{\mathbf{V}}(s) d s, \mathbf{z}_{\Omega} \triangleq \int_{0}^{t} \widetilde{\mathbf{\Omega}}(s) d s$, and

$$
\left\{\begin{array}{l}
\overline{\mathbf{F}}_{d r} \triangleq-\left(\mathbf{D}_{V l}+|\mathbf{V}| \mathbf{D}_{V q}\right) \mathbf{V}_{r} \\
\boldsymbol{\Gamma}_{d r} \triangleq-\left(\mathbf{D}_{\Omega l}+|\boldsymbol{\Omega}| \mathbf{D}_{\Omega q}\right) \boldsymbol{\Omega}_{r}
\end{array}\right.
$$

Assume that the disturbance terms $\boldsymbol{\Delta}_{F}$ and $\boldsymbol{\Delta}_{\Gamma}$ are constant. Let $\mathbf{V}_{r} \triangleq \mathbf{V}_{C r}-\boldsymbol{\Omega}_{r} \times \mathbf{r}_{C}$, with $\mathbf{V}_{C r}$ defined by Proposition 11 Then, the following properties hold:

1) If $\boldsymbol{\Omega}_{r}$ is defined by (21) (c.f. Proposition 2), the equilibrium $\left(\mathbf{V}, \boldsymbol{\Omega}, \mathbf{z}_{V}, \mathbf{z}_{\Omega}\right)=\left(\mathbf{V}_{r}, \boldsymbol{\Omega}_{r}, \mathbf{z}_{V}^{\star}, \mathbf{z}_{\Omega}^{\star}\right)$ (with $\mathbf{z}_{V}^{\star} \triangleq$ $\mathbf{K}_{i V}^{-1} \boldsymbol{\Delta}_{F}$ and $\mathbf{z}_{\Omega}^{\star} \triangleq \mathbf{K}_{i \Omega}^{-1} \boldsymbol{\Delta}_{\Gamma}$ ) of the controlled system is globally asymptotically stable (GAS) and locally exponentially stable (LES).

2) If $\boldsymbol{\Omega}_{r}$ is defined by (25)+24) (c.f. Theorem (1), then, the controlled system has two equilibria $\left(\mathbf{V}, \boldsymbol{\Omega}, \mathbf{R}^{\top} \mathbf{e}_{g}, \mathbf{z}_{V}, \mathbf{z}_{\Omega}\right)=\left(\mathbf{V}_{r}, \boldsymbol{\Omega}_{r}, \pm \mathbf{e}_{g}, \mathbf{z}_{V}^{\star}, \mathbf{z}_{\Omega}^{\star}\right)$. The "desired" equilibrium $\left(\mathbf{V}, \boldsymbol{\Omega}, \mathbf{R}^{\top} \mathbf{e}_{g}, \mathbf{z}_{V}, \mathbf{z}_{\Omega}\right)=$ $\left(\mathbf{V}_{r}, \boldsymbol{\Omega}_{r}, \mathbf{e}_{g}, \mathbf{z}_{V}^{\star}, \mathbf{z}_{\Omega}^{\star}\right)$ is almost-GAS and LES, whereas the "undesired" equilibrium $\left(\mathbf{V}, \boldsymbol{\Omega}, \mathbf{R}^{\top} \mathbf{e}_{g}, \mathbf{z}_{V}, \mathbf{z}_{\Omega}\right)=\left(\mathbf{V}_{r}, \boldsymbol{\Omega}_{r},-\mathbf{e}_{g}, \mathbf{z}_{V}^{\star}, \mathbf{z}_{\Omega}^{\star}\right)$ is unstable. Thus, $\left(\mathbf{V}, \boldsymbol{\Omega}, \mathbf{R}^{\top} \mathbf{e}_{g}, \mathbf{z}_{V}, \mathbf{z}_{\Omega}\right)$ converges to the desired equilibrium for almost all initial conditions.

For readability purposes, the proposed control architecture is depicted in Fig. 2.

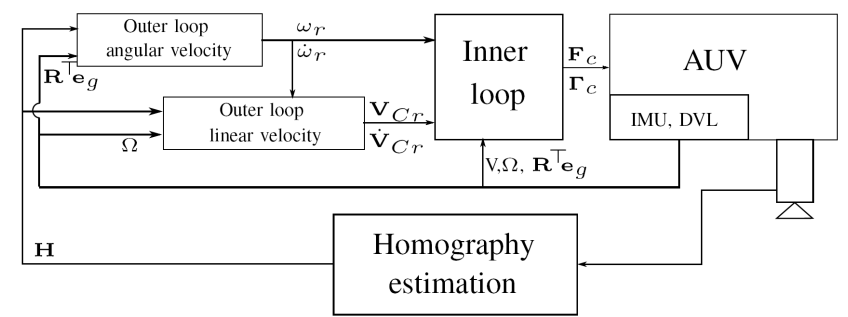

Fig. 2: Block diagram of the proposed HBVS controller (outerloop as in Theorem 1 and inner-loop as in Proposition 3).

\section{A. Adaptations to almost fully-actuated AUVs}

In practice, the roll motion may not be actuated by conception (i.e. $\Gamma_{c, 1} \equiv 0$ ) and is, thus, left passively stabilised by restoring and dissipative roll moments. We will show that the inner-loop controller in Proposition 3 can be adapted to such a situation, of course under some reasonable assumptions. First, we assume that the AUV is well conceived so that its CoM is located below its $\mathrm{CoB}$ and along the $\vec{e}_{3}^{b}$-axis. We also assume that the reference image is taken when the AUV stays in a horizontal plane so that $\mathbf{e}_{g} \equiv \mathbf{e}_{3}$. Finally, since the roll 
motion is not actively actuated and the conception normally ensures an effective passive roll stabilisation, it is reasonable to assume that roll motion is negligible, i.e. $\phi \approx 0$ and $\omega_{1} \approx 0$. Therefore, the expression (25) of $\boldsymbol{\Omega}_{r}$ is simplified to

$$
\boldsymbol{\Omega}_{r}=k_{u}\left(\mathbf{e}_{1}^{\top} \mathbf{R}^{\top} \mathbf{e}_{3}\right) \mathbf{e}_{2},+\omega_{r} \mathbf{e}_{3},
$$

where its first component $\omega_{1 r}$ is null. Then, using the assumption $\omega_{1}=0$ one deduces that the first component of $\widetilde{\Omega}$ is also null, i.e. $\tilde{\omega}_{1}=0$.

For later use, let $\mathbf{x}_{2,3} \in \mathbb{R}^{2}$ denote the vector of last two components of any $\mathrm{x} \in \mathbb{R}^{3}$.

Corollary 1. Consider the system dynamics 27a-27b and apply the following controller:

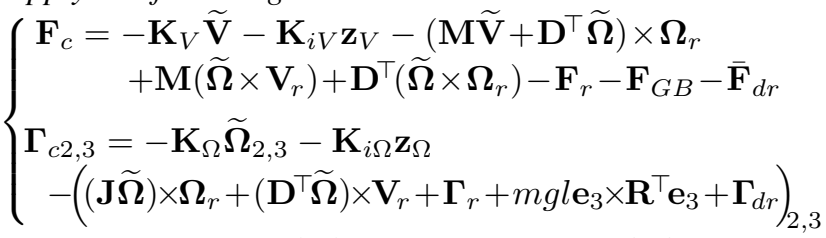

with $\mathbf{K}_{V}, \mathbf{K}_{i V} \in \mathbb{R}^{3 \times 3}$ and $\mathbf{K}_{\Omega}, \mathbf{K}_{i \Omega} \in \mathbb{R}^{2 \times 2}$ some positive diagonal gain matrices, $\mathbf{z}_{V} \triangleq \int_{0}^{t} \widetilde{\mathbf{V}}(s) d s, \mathbf{z}_{\Omega} \triangleq \int_{0}^{t} \widetilde{\mathbf{\Omega}}_{2,3}(s) d s$, and $\overline{\mathbf{F}}_{d r}, \boldsymbol{\Gamma}_{d r}$ defined by [29]. Define $\mathbf{V}_{r} \triangleq \mathbf{V}_{C r}-\boldsymbol{\Omega}_{r} \times \mathbf{r}_{C}$, with $\mathbf{V}_{C r}$ given by Proposition 1 and $\boldsymbol{\Omega}_{r}$ defined by $\left.(30)+24\right]$ (c.f. Theorem 17). Assume that the disturbance terms $\boldsymbol{\Delta}_{F}$ and $\Delta_{\Gamma}$ are constant. The roll motion is assumed to be negligible so that $\omega_{1}=\phi=0$. Then, the controlled system has two equilibria $\left(\mathbf{V}, \boldsymbol{\Omega}, \mathbf{R}^{\top} \mathbf{e}_{g}, \mathbf{z}_{V}, \mathbf{z}_{\Omega}\right)=\left(\mathbf{V}_{r}, \boldsymbol{\Omega}_{r}, \pm \mathbf{e}_{g}, \mathbf{z}_{V}^{\star}, \mathbf{z}_{\Omega}^{\star}\right)$, with $\mathbf{z}_{V}^{\star} \triangleq \mathbf{K}_{i V}^{-1} \boldsymbol{\Delta}_{F}$ and $\mathbf{z}_{\Omega}^{\star} \triangleq \mathbf{K}_{i \Omega}^{-1} \boldsymbol{\Delta}_{\Gamma 2,3}$. The "desired" equilibrium $\left(\mathbf{V}, \boldsymbol{\Omega}, \mathbf{R}^{\top} \mathbf{e}_{g}, \mathbf{z}_{V}, \mathbf{z}_{\Omega}\right)=\left(\mathbf{V}_{r}, \boldsymbol{\Omega}_{r}, \mathbf{e}_{g}, \mathbf{z}_{V}^{\star}, \mathbf{z}_{\Omega}^{\star}\right)$ is almost-GAS and LES, whereas the "undesired" equilibrium $\left(\mathbf{V}, \boldsymbol{\Omega}, \mathbf{R}^{\top} \mathbf{e}_{g}, \mathbf{z}_{V}, \mathbf{z}_{\Omega}\right)=\left(\mathbf{V}_{r}, \boldsymbol{\Omega}_{r},-\mathbf{e}_{g}, \mathbf{z}_{V}^{\star}, \mathbf{z}_{\Omega}^{\star}\right)$ is unstable. Thus, $\left(\mathbf{V}, \boldsymbol{\Omega}, \mathbf{R}^{\top} \mathbf{e}_{g}, \mathbf{z}_{V}, \mathbf{z}_{\Omega}\right)$ converges to the desired equilibrium for almost all initial conditions.

The proof straightforwardly follows the same lines as the proof of Proposition 3 and is given in Appendix $\mathrm{H}$

\section{Simulation RESUlts}

The proposed control approach has been tested by simulations, with a realistic model of a fully-actuated AUV. The

\begin{tabular}{|c|c|}
\hline Specification & Numerical value \\
\hline Water density $\rho_{f}\left[\mathrm{~kg} / \mathrm{m}^{3}\right]$ & 1000 \\
\hline Mass $m[k g]$ & 160 \\
\hline Volume $V\left[\mathrm{~m}^{3}\right]$ & 0.1616 \\
\hline$l[m]$ & 0.15 \\
\hline $\mathbf{r}_{C}[m]$ & $\left.\begin{array}{ll}0 & 0.5\end{array}\right]^{\top}$ \\
\hline & $\left.\begin{array}{ll}5 & 10\end{array}\right]$ \\
\hline $\mathbf{J}=\mathbf{J}_{0}+\mathbf{M}_{A}^{22}\left[k g \cdot \mathrm{m}^{2}\right]$ & 110 \\
\hline & 10 \\
\hline & $15^{-4}$ \\
\hline $\mathbf{M}_{A}^{11}[k g]$ & 187 \\
\hline & 525 \\
\hline & 10 \\
\hline $\mathbf{M}_{\Delta}^{12}=\mathbf{M}_{\Lambda}^{21 \top}[k g . m]$ & 10 \\
\hline & 0.5 \\
\hline $\mathbf{D}_{V l}\left[k g . s^{-1}\right]$ & $\operatorname{diag}(1,1.2,1.4)$ \\
\hline $\mathbf{D}_{V q}\left[\mathrm{~kg} \cdot \mathrm{m}^{-1}\right]$ & $\operatorname{diag}(15,40,70)$ \\
\hline $\mathbf{D}_{\Omega l}\left[\mathrm{~kg} \cdot \mathrm{m}^{2} \cdot \mathrm{s}^{-1}\right]$ & $\operatorname{diag}(0.3,0.2,0.4)$ \\
\hline $\mathbf{D}_{\Omega q}[N . m]$ & $\operatorname{diag}(3,2,4)$ \\
\hline
\end{tabular}
physical parameters are given in Tab. I]

TABLE I: Specifications of the simulated AUV.

The objective of this simulation section is to illustrate the performance and robustness of our HBVS control approach compared to the kinematic-based control approach proposed in [5]. Since the difference only concerns the outer-loop level, we apply the same inner-loop controller 28 proposed in Proposition 3 As for the outer-loop level, the three following outer-loop controllers, that define the reference velocities $\mathbf{V}_{C r}$ and $\boldsymbol{\Omega}_{r}$ and their derivative, are used for comparison purposes:

- Outer-loop controller $\mathbf{1}$ [5]: $\mathbf{V}_{C r}$ and $\boldsymbol{\Omega}_{r}$ are computed according to 13 . The feed-forward terms $\dot{\mathbf{V}}_{C r}$ and $\dot{\boldsymbol{\Omega}}_{r}$ are simply set equal to zero in the inner-loop controller.

- Outer-loop controller 2 (Proposition 11+Proposition 2): $\mathbf{V}_{C r}$ is computed using $(20)+(19)$ as in Proposition 1 . while $\boldsymbol{\Omega}_{r}$ is computed using 22] as in Proposition 2.

- Outer-loop controller 3 (Proposition 11+Theorem 1): $\mathbf{V}_{C r}$ is computed similarly to the outer-loop controller 2 , but $\boldsymbol{\Omega}_{r}$ is computed using 25] as in Theorem 2

The homography matrix $\mathbf{H}$ is directly calculated according to (6), with $d^{\star}=1(m)$ and $\mathbf{n}^{\star}=$ $[-0.4924,0.1736,-0.8529]^{\top}=-\mathbf{R}_{\left\{\frac{\pi}{18}, \frac{\pi}{6}, 0\right\}} \mathbf{e}_{3}$. The vector $\mathbf{m}^{\star}$ involved in the computations of all these outer-loop controllers is given by $\mathbf{m}^{\star}=-\mathbf{e}_{3}\left(\neq \mathbf{n}^{\star}\right)$. One verifies that $\mathbf{n}^{\star \top} \mathbf{m}^{\star}=0.8529>0$ so that Assumption 1 is satisfied.

The gain matrices $\mathbf{K}_{V}$ and $\mathbf{K}_{\Omega}$ of the inner-loop controller (28) are given by ${ }^{2}$

$$
\left\{\begin{array}{l}
\mathbf{K}_{V}=\operatorname{diag}(233.7,347.4,660) \\
\mathbf{K}_{\Omega}=\operatorname{diag}(159.8,199.6,150) \\
\mathbf{K}_{i V}=0.1 \mathbf{K}_{V}, \mathbf{K}_{i \Omega}=0.1 \mathbf{K}_{\Omega}
\end{array}\right.
$$

To test the robustness of the proposed inner-loop controller, we make use of the "erroneous" estimated parameters instead of the real values:

$$
\left\{\begin{array}{l}
\hat{\mathbf{J}}=\operatorname{diag}(80,100,75)\left(\mathrm{kg} \cdot \mathrm{m}^{2}\right), \\
\hat{\mathbf{M}}=m \mathbf{I}_{3}+\hat{\mathbf{M}}_{A}^{11}=\operatorname{diag}(250,368,660)(\mathrm{kg}), \\
\hat{\mathbf{D}}=m \mathbf{e}_{3 \times}=24 \mathbf{e}_{3 \times}(\mathrm{kg} \cdot \mathrm{m}) .
\end{array}\right.
$$

Moreover, we introduce a constant current velocity $\mathbf{v}_{f}=$ $[0,0.5,0]^{\top}(\mathrm{m} / \mathrm{s})$ so that the disturbance terms $\boldsymbol{\Delta}_{F}$ and $\boldsymbol{\Delta}_{\Gamma}$ are not negligible, showing the need of integral actions.

\section{A. Poor performance of the outer-loop controller 1}

We have carried out extensive simulations using the outerloop controller 1 [5], with different set of gains $\left(k_{p}, k_{\Theta}\right)$ along with different initial conditions. We observe that the lower the gains $\left(k_{p}, k_{\Theta}\right)$ the larger the domain of stability is, of course at the cost of slow convergence rate. For instance, Figs. 3 - 6 show the evolutions of $\mathbf{e}_{p}$ and $\mathbf{e}_{\Theta}$, using either small gains $\left(k_{p}=k_{\Theta}=0.5\right)$ or higher gains $\left(k_{p}=1.5, k_{\Theta}=2.5\right)$, and for both cases of small and large initial (position and orientation) errors. From Figs. 3 and 4 , one observes that when the initial errors are small (i.e. $\mathbf{p}_{C}(0)=[-0.5,-0.6,0.8]^{\top}(m)$, $\left.\mathbf{R}(0)=\mathbf{R}_{\left\{\frac{\pi}{18},-\frac{\pi}{18}, \frac{\pi}{6}\right\}}\right)$, the visual errors $\mathbf{e}_{p}$ and $\mathbf{e}_{\Theta}$ converge to zero for both cases of $\left(k_{p}=k_{\Theta}=0.5\right)$ and $\left(k_{p}=1.5, k_{\Theta}=2.5\right)$. The convergence rate for the case of $\left(k_{p}=1.5, k_{\Theta}=2.5\right)$ is clearly faster than the case of $\left(k_{p}=k_{\Theta}=0.5\right)$. However, when the initial errors are large (i.e. $\mathbf{p}_{C}(0)=[-4,-3,-5]^{\top}(m), \mathbf{R}(0)=\mathbf{R}_{\left\{\frac{\pi}{6},-\frac{\pi}{18}, \pi\right\}}$ ), the controlled system, with $\left(k_{p}=1.5, k_{\Theta}=2.5\right)$, tends to be

\footnotetext{
${ }^{2}$ These gains are tuned based on classical pole placement technique, with two triple negative real poles equal to -1 and -2 , on the linearised closedloop system 27. for the particular case where $\mathbf{V}_{r} \equiv \boldsymbol{\Omega}_{r} \equiv \mathbf{v}_{f} \equiv \mathbf{0}$.
} 

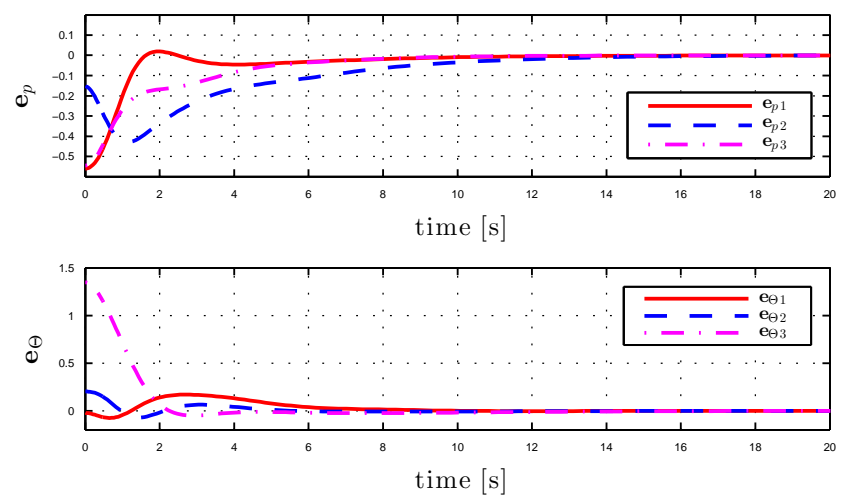

Fig. 3: $\mathbf{e}_{p}$ and $\mathbf{e}_{\Theta}$ vs. time. Outer-loop controller 1 with small gains $k_{p}=k_{\Theta}=0.5$ and small initial errors $\mathbf{p}_{C}(0)=$ $[-0.5,-0.6,0.8]^{\top}(m), \mathbf{R}(0)=\mathbf{R}_{\left\{\frac{\pi}{18},-\frac{\pi}{18}, \frac{\pi}{6}\right\}}$.
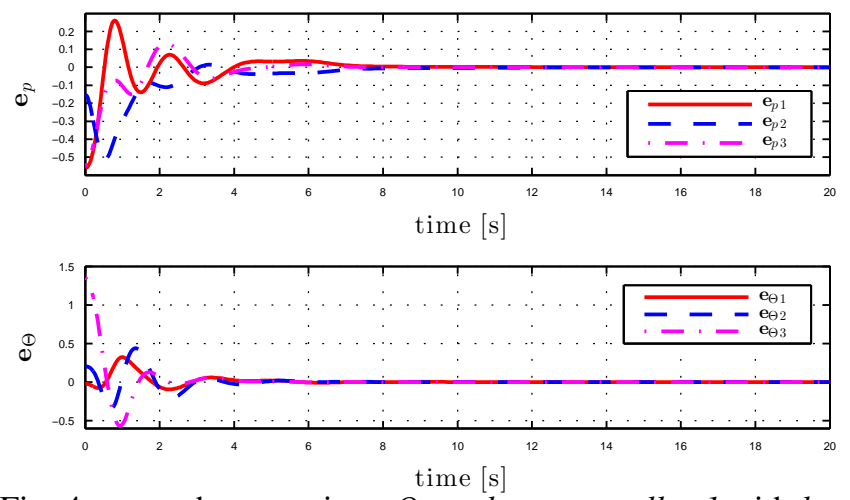

Fig. 4: $\mathbf{e}_{p}$ and $\mathbf{e}_{\Theta}$ vs. time. Outer-loop controller 1 with large gains $k_{p}=1.5, k_{\Theta}=2.5$ and small initial errors $\mathbf{p}_{C}(0)=$ $[-0.5,-0.6,0.8]^{\top}(m), \mathbf{R}(0)=\mathbf{R}_{\left\{\frac{\pi}{18},-\frac{\pi}{18}, \frac{\pi}{6}\right\}}$.

unstable as observed in Fig. 6 One also observes from Fig. 5 that for the case of large initial errors and small gains $\left(k_{p}=k_{\Theta}=0.5\right)$, although $\mathbf{e}_{p}$ and $\mathbf{e}_{\Theta}$ still converge to zero, their evolutions are quite oscillating. Moreover, the AUV makes a large roll motion (attaining nearly $180(\mathrm{deg})$ ) before converging to zero, which is not desirable in practice. The poor performance of this "hierarchical" kinematic-based controller, especially in the case of large initial errors and/or in the case of high gains, is not surprising since its design and stability analysis are only established on local basis.

\section{B. Improved performance of the proposed outer-loop con- trollers}

We now report the improved performance of the proposed outer-loop controllers, i.e. the outer-loop controllers 2 and 3. In order to show that it is possible to provide fast convergence rate without any influence on the stability domain, the gains involved in the outer-loop controllers 2 and 3 are chosen so that the local convergence rate of these controllers is similar to the one of the outer-loop controller 1 with $k_{p}=1.5, k_{\Theta}=2.5$. Consequently, the gains and parameters involved in the outerloop controller 2 are given by $?^{3}$

$$
\begin{aligned}
& k_{p_{1}}=1.5, k_{p 2}=6, k_{z}=10, \Delta=5, \nabla=0.5, \\
& k_{\Theta 1}=2.5, k_{\Theta 2}=3.162, \Delta_{\omega}=2,
\end{aligned}
$$

\footnotetext{
${ }^{3}$ Gain tuning is also based on the classical pole placement technique for the linearised system about the corresponding equilibrium and by imposing $\mathbf{V}_{C} \equiv \mathbf{V}_{C r}$ and $\boldsymbol{\Omega} \equiv \boldsymbol{\Omega}_{r}$
}
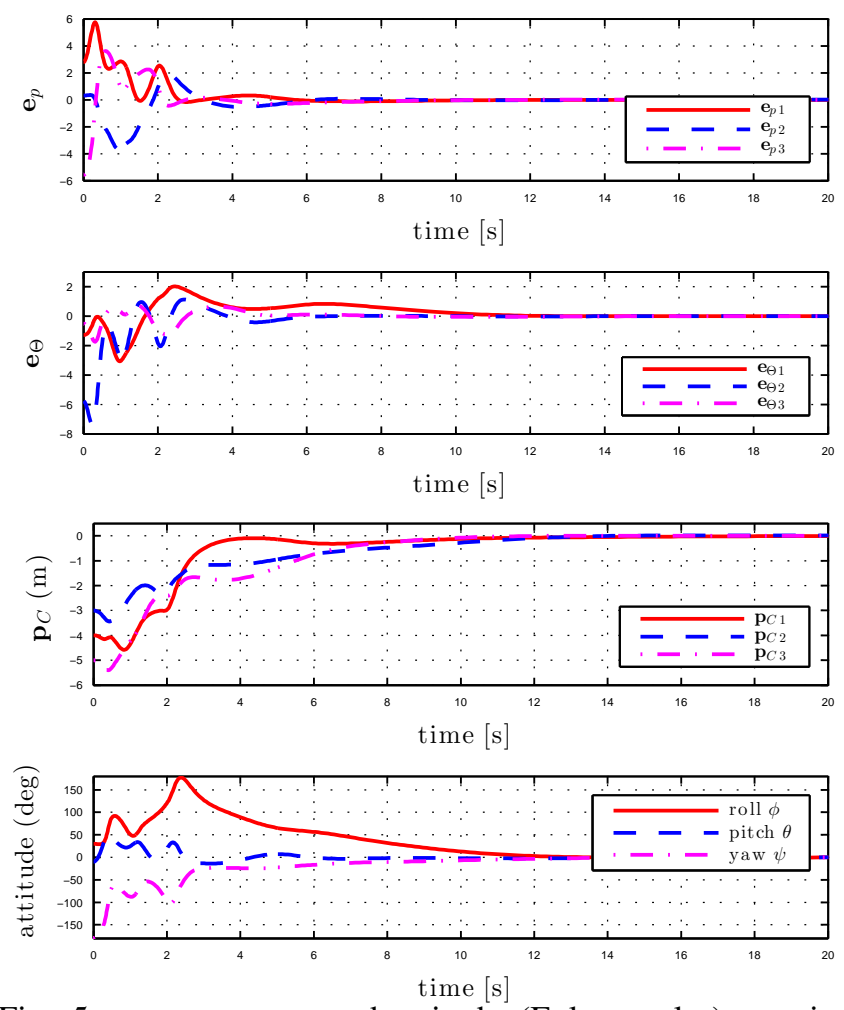

Fig. 5: $\mathbf{e}_{p}, \mathbf{e}_{\Theta}, \mathbf{p}_{C}$ and attitude (Euler angles) vs. time. Outer-loop controller 1 with small gains $k_{p}=k_{\Theta}=0.5$ and large initial errors $\mathbf{p}_{C}(0)=[-4,-3,-5]^{\top}(m), \mathbf{R}(0)=$ $\mathbf{R}_{\left\{\frac{\pi}{6},-\frac{\pi}{18}, \pi\right\}}$.
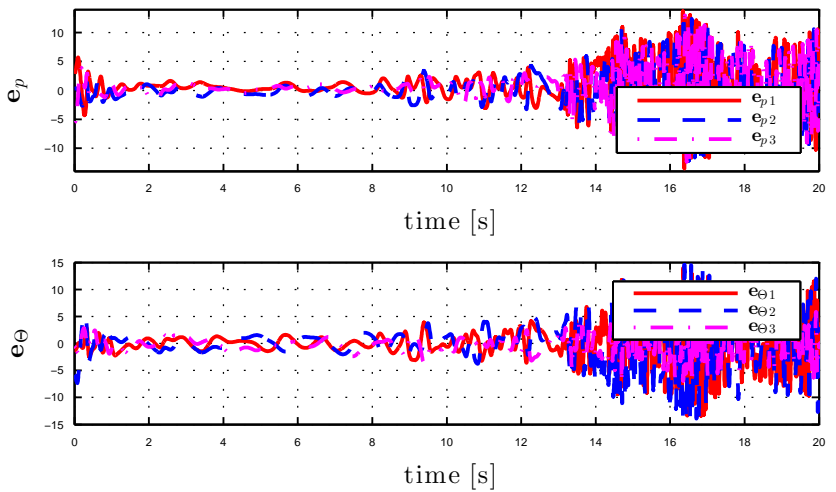

Fig. 6: $\mathbf{e}_{p}$ and $\mathbf{e}_{\Theta}$ vs. time. Outer-loop controller 1 with large gains $k_{p}=1.5, k_{\Theta}=2.5$ and large initial errors $\mathbf{p}_{C}(0)=$ $[-4,-3,-5]^{\top}(m), \mathbf{R}(0)=\mathbf{R}_{\left\{\frac{\pi}{6},-\frac{\pi}{18}, \pi\right\}}$.

while those involved in the outer-loop controller 3 are

$$
\begin{aligned}
& k_{p_{1}}=1.5, k_{p 2}=6, k_{z}=10, \Delta=5, \nabla=0.5, \\
& k_{\Theta 1}=5, k_{\Theta 2}=3.162, \Delta_{\omega}=1, k_{u}=2.5 .
\end{aligned}
$$

Figs. 7 and 8 show the evolutions of $\mathbf{e}_{p}, \mathbf{e}_{\Theta}, \mathbf{p}_{C}$, and the AUV's Euler angles (i.e. orientation), for the outer-loop controllers 2 and 3 respectively, with the same large initial (position and orientation) errors already used for testing the outer-loop controller 1, i.e. $\mathbf{p}_{C}(0)=[-4,-3,-5]^{\top}(\mathrm{m})$, $\mathbf{R}(0)=\mathbf{R}_{\left\{\frac{\pi}{6},-\frac{\pi}{18}, \pi\right\}}$. One can clearly see a net improvement of performance w.r.t. the outer-loop controller 1. Indeed, from these figures very fast convergence rate and much less oscillations w.r.t. the case of the outer-loop controller 1 (Fig. 5 can be observed. In particular, for the outer-loop controller 

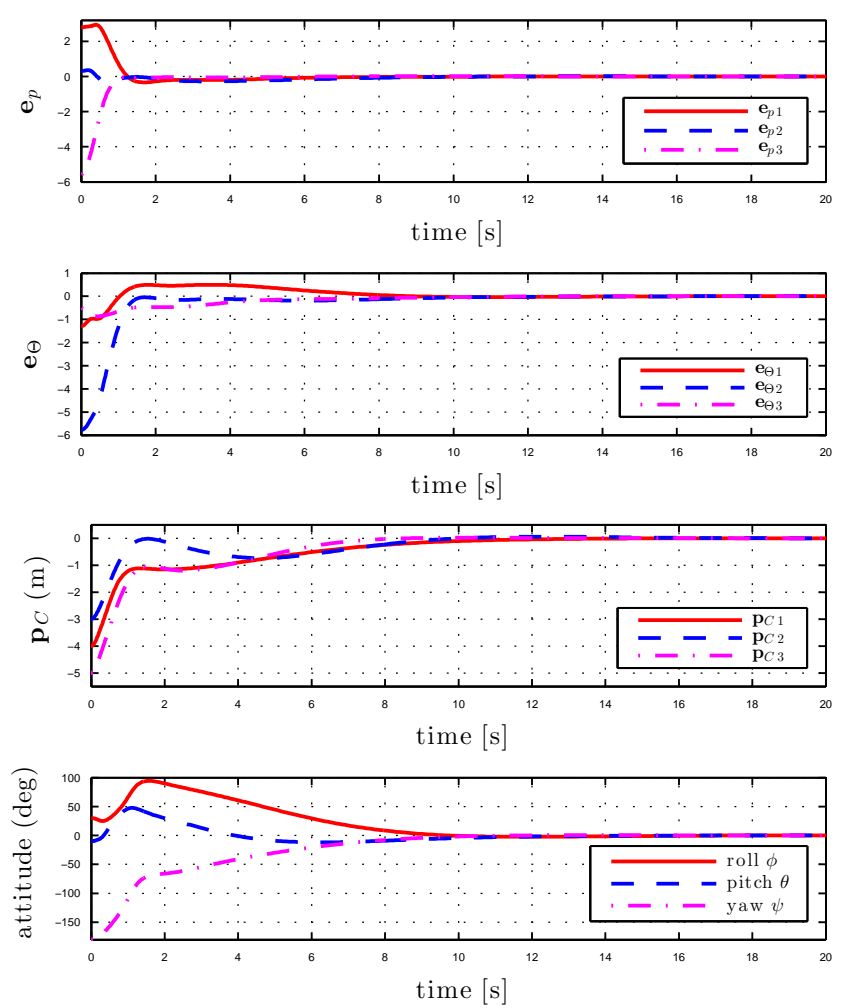

Fig. 7: $\mathbf{e}_{p}, \mathbf{e}_{\Theta}, \mathbf{p}_{C}$ and attitude (Euler angles) vs. time. Outer-loop controller 2 with large initial errors $\mathbf{p}_{C}(0)=$ $[-4,-3,-5]^{\top}(m), \mathbf{R}(0)=\mathbf{R}_{\left\{\frac{\pi}{6},-\frac{\pi}{18}, \pi\right\}}$.
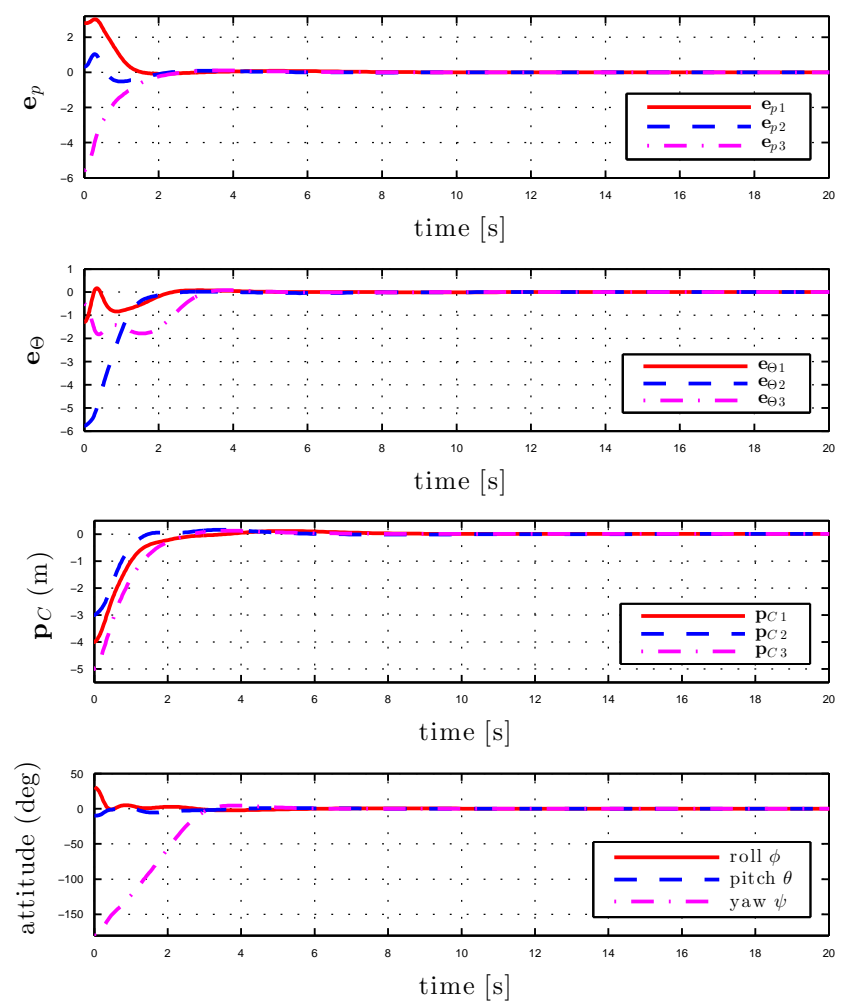

Fig. 8: $\mathbf{e}_{p}, \mathbf{e}_{\Theta}, \mathbf{p}_{C}$ and attitude (Euler angles) vs. time. Outer-loop controller 3 with large initial errors $\mathbf{p}_{C}(0)=$ $[-4,-3,-5]^{\top}(m), \mathbf{R}(0)=\mathbf{R}_{\left\{\frac{\pi}{6},-\frac{\pi}{18}, \pi\right\}}$.

3, thanks to the use of the gravity direction measurement (i.e. $\mathbf{R}^{\top} \mathbf{e}_{3}$ ) the roll and pitch Euler angles quickly converge near to zero without growing large (see Fig. 8), unlike the cases of the outer-loop controllers 1 and 2 . This is the behaviour that we find very satisfactory, since in practice it is often desirable to maintain small roll and pitch angles.

\section{EXPERIMENTAL RESULTS}

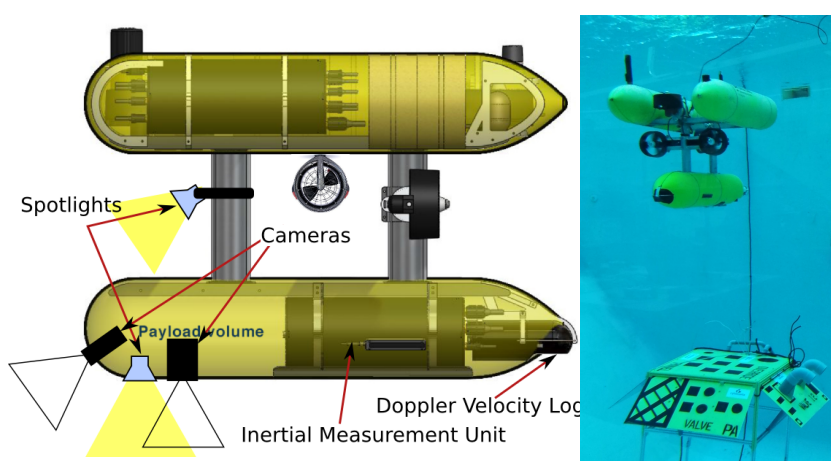

Fig. 9: Girona 500 AUV and experimental setup.

The experiments have been performed on an AUV Girona 500 developed by the Underwater Vision and Robotics Center (Girona, Spain) [36] (see Fig. 9]. The overall dimensions of the vehicle are $1 \times 1 \times 1.5[\mathrm{~m}]$ of height, width, and length. Its weight is approximately $160[\mathrm{~kg}]$ in air. In addition to basic sensors such as an IMU and a DVL, the AUV is also equipped with two cameras: a Fire-Wire downward-looking camera (along the direction $\vec{e}_{3}^{b}$ ) and a PAL forward-looking camera $\left(55[\mathrm{deg}]\right.$ rotation around $\left.\vec{e}_{2}^{b}\right)$, both providing color images at about $5-7[\mathrm{~Hz}]$. The Girona $500 \mathrm{AUV}$ is equipped with two vertical thrusters for heave and pitch actuations, two horizontal thrusters for yaw and surge actuations, and one lateral thruster for sway actuation. The roll motion is, thus, left passively stabilised.

To emulate an inspection of an underwater infrastructure, we placed in a pool a mockup of a realistic subsea manifold whose size is approximately $2\left[\mathrm{~m}^{2}\right]$ (see Fig. 9). For each camera (i.e. downward- or forward-looking), reference images have been collected in teleoperation mode at different poses of the UAV around the mockup manifold, so that the whole mockup can be monitored. A region of interest corresponding to a planar target in each reference image has been selected. ROS middleware is used to provide transparent support for transferring images from camera in low-bandwidth compressed formats. A bridge between ROS images and OpenCV is also used to obtain in real-time the estimated homography matrix using OpenCV functions integrated into ROS communication graph.

For each the camera configuration (i.e. downward- or forward-looking), the AUV is initially placed in teleoperation mode to some pose so that the camera can view the mockup manifold. Then, the proposed controller is activated to drive the vehicle to the desired pose based on the onboard computed homography matrix. When the norms of the visual errors $\mathbf{e}_{\Theta}$ and $\mathbf{e}_{p}$ are less than some given small thresholds, another image previously collected is then used as the next reference image. The control gains and other parameters involved in the computation of the control inputs (Proposition 1 t Theorem 11+Corollary 1) are given as follows:

- $\hat{\mathbf{J}}, \hat{\mathbf{M}}, \hat{\mathbf{D}}$ given by (33); 


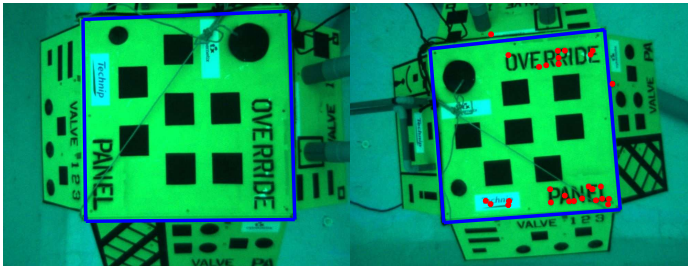

(a) Reference image

(b) $\mathrm{t}=73 \mathrm{~s}$

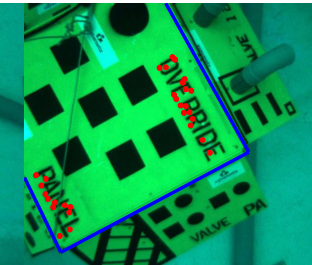

(c) $\mathrm{t}=84 \mathrm{~s}$

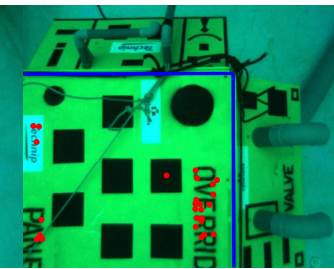

(d) $\mathrm{t}=90 \mathrm{~s}$

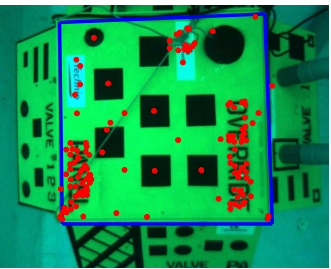

(e) $\mathrm{t}=110 \mathrm{~s}$

Fig. 10: Mission 1, reference image and current images with downward-looking camera.

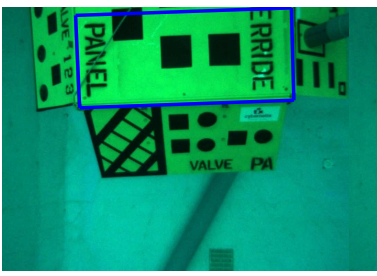

(a) Reference image

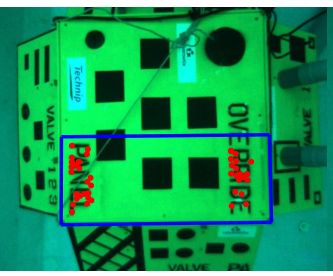

(b) $\mathrm{t}=111 \mathrm{~s}$

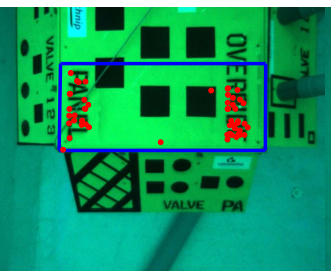

(c) $\mathrm{t}=115 \mathrm{~s}$

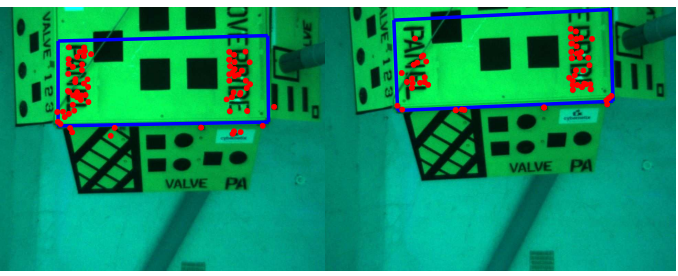

(d) $\mathrm{t}=126 \mathrm{~s}$ (e) $\mathrm{t}=133 \mathrm{~s}$

Fig. 11: Mission 2, reference image and current images with downward-looking camera.

- $\mathbf{K}_{V}=\operatorname{diag}(233.7,347.4,660), \mathbf{K}_{\Omega}=\operatorname{diag}(199.6,150)$, $\mathbf{K}_{i V}=0.1 \mathbf{K}_{V}, \mathbf{K}_{i \Omega}=0.1 \mathbf{K}_{\Omega}$

- $k_{p 1}=0.3, k_{p 2}=1.2, k_{z}=2, \Delta=10, \nabla=5$;

- $k_{\Theta 1}=0.5, k_{\Theta 2}=1, \Delta_{\omega}=2, k_{u}=1$;

- $\mathbf{m}^{\star}=-\mathbf{e}_{3}$ and $\mathbf{m}^{\star}=\left[-\sin \left(\frac{55 \pi}{180}\right), 0,-\cos \left(\frac{55 \pi}{180}\right)\right]^{\top}$ for downward- and forward-looking camera, respectively; $\mathbf{r}_{C}=[0.5,0,0.5]^{\top}[m]$ for both cases.

In the following experimental results corresponding to both camera configurations will be reported. Due to space limitation, only brief but representative parts of total results are presented. However, the willing reader is invited to view a video clip showing the whole 8-minute experiments (see also multimedia attachment) at https://youtu.be/BD5nEZWJRKA.

\section{A. Experiment with downward-looking camera}

Experimental results corresponding to the two consecutive reference images given in Figs. 10a and 11a, that we call respectively "Mission 1" and "Mission 2", are reported on Figs. 10-15. They correspond to the period between $22[s]$ and $38[s]$ of the video. The reference images and current images that are taken at different time instants during the transition are shown on Figs. 10 and 11. The blue rectangles enclose the planar targets of interest where feature points (i.e. red points in Figs. 10b 10e and 11b 11e are sought and matched with the reference ones. The convergence of the current image to the reference image can be clearly observed. The visual errors $\mathbf{e}_{p}$ and $\mathbf{e}_{\Theta}$, along with their norms that are used for switching between the reference images, are shown in Figs. 12 and 13 . One observes that these error quantities converge to small values despite the large initial error in yaw (for Mission 1), the behaviour that we find quite satisfactory. As these errors are computed from the homography matrix, one can equally see the effect of imperfect homography matrix estimated from image processing. One can also observe some oscillations during the convergence. This is essentially due to update rate (too low in the carried out experiments) that leads to unavoidable delay (larger than $150 \mathrm{~ms}$ ) that degrades the controlled-system performance. Fig. 14 presents the outputs
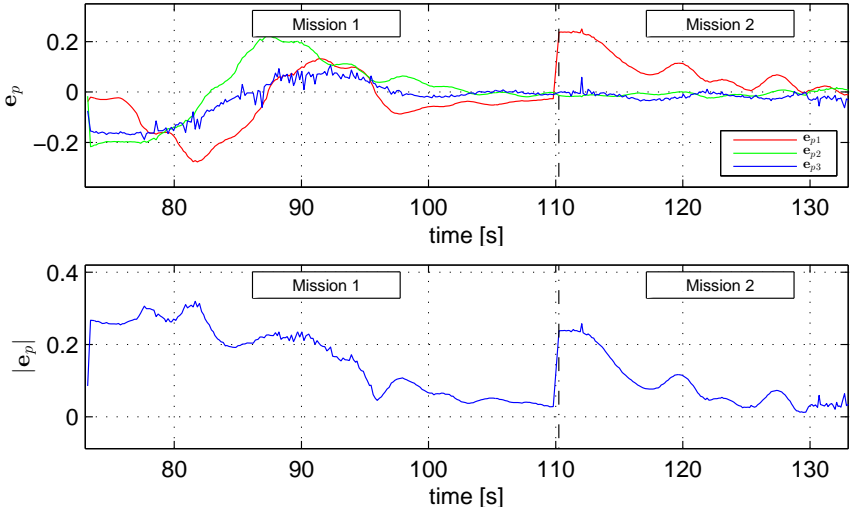

Fig. 12: $\mathbf{e}_{p}$ and $\left|\mathbf{e}_{p}\right|$ vs. time (downward-looking camera).
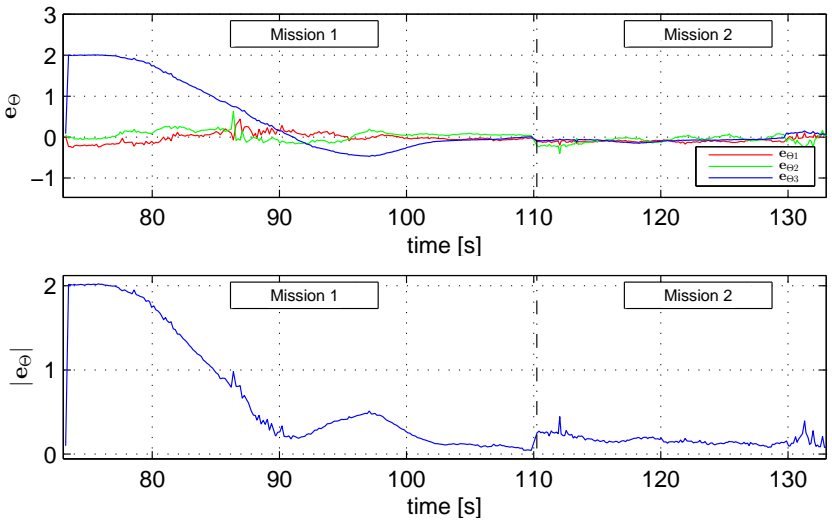

Fig. 13: $\mathbf{e}_{\Theta}$ and $\left|\mathbf{e}_{\Theta}\right|$ vs. time (downward-looking camera).

of the outer-loop controller. One observes from Fig. 14 that during Mission 2 (i.e. after $110[s]$ ) only the first component of the reference translational velocity (i.e. $V_{r, 1}$ ) is significantly involved. This, in fact, corresponds to a pure translation along the $\vec{e}_{1}^{b}$-axis. On the other hand, Fig. 15 shows the control force and torque vectors computed from the inner-loop control. One remarks that the third component of the control force vector (i.e. $F_{c, 3}$ ) ultimately remains far from zero (i.e. $\left.F_{c, 3} \approx 60[N]\right)$ since the Girona 500 is positively buoyant. The pitch torque control $\Gamma_{c, 2}$ approximately converges to $5[N . m]$ 

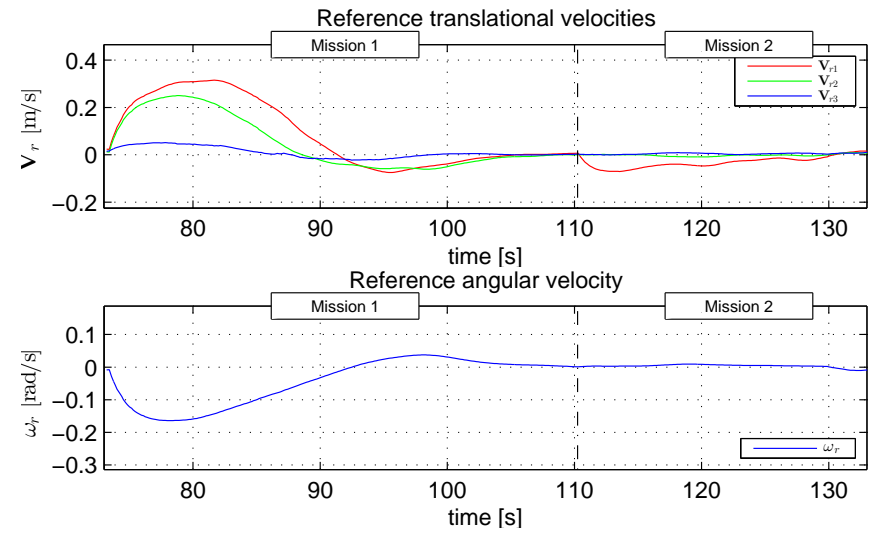

Fig. 14: Reference translational and angular velocities vs. time (downward-looking camera).
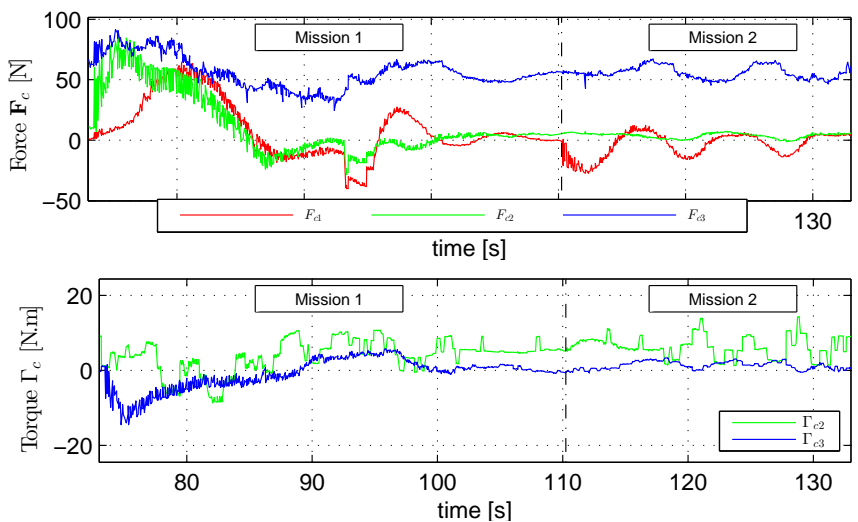

Fig. 15: Control force and torque vs. time (downward-looking camera).

between $100[s]$ and $110[s]$ (Mission 1). This can be explained by the fact that the gravitational force does not pass through the center of buoyancy (i.e. $\overrightarrow{B G}$ is not parallel to $\vec{e}_{3}^{b}$ ) and, thus, induces a parasite torque that is compensated by the integral term $\mathbf{z}_{\Omega}$ involved in the inner-loop controller. This also justifies the robustness of the proposed control approach w.r.t. unavoidable model uncertainties.

\section{B. Experiment with forward-looking camera}

Experimental results related to the reference image given in Fig. 18a using the forward-looking camera are reported on Figs. 16 18. They correspond to the period between 90 [s] and 109[s] of the video. The reference image and current images during the transition are given in Figs. $18 \mathrm{~b}-18 \mathrm{e}$ showing the convergence of the current image to the reference one. Similarly, the convergence of the visual errors terms $\mathbf{e}_{p}, \mathbf{e}_{\Theta}$, and their norms to small values can be observed from Figs. 16 and 17 after a short transient period.

In conclusion the experimental results for the overall control approach (i.e. inner- and outer-loop controls) with both camera configurations are quite convincing despite the fact that the vehicle's physical parameters are not well known and that the vehicle is only almost fully-actuated (i.e. roll actuation is not active).

\section{CONCLUSIONS}

We have proposed an inertial-aided image-based visual servo controller for the stabilisation of (almost) fully-actuated
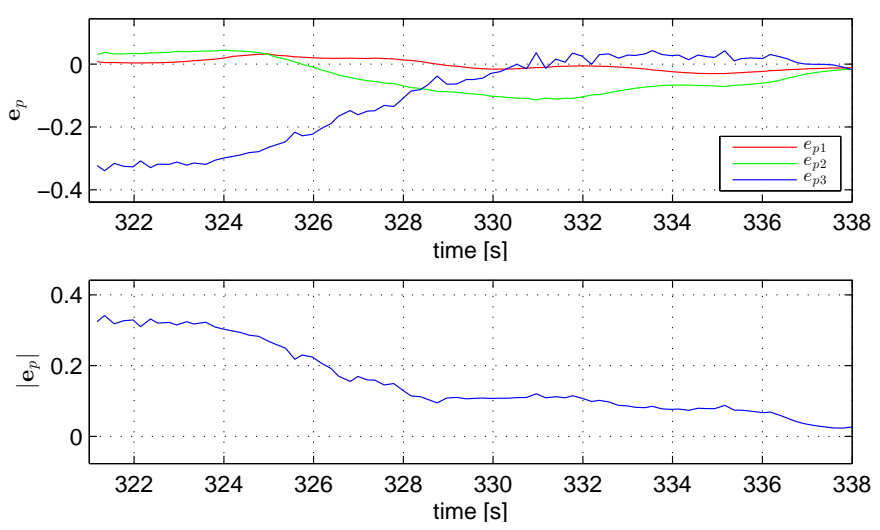

Fig. 16: $\mathbf{e}_{p}$ and $\left|\mathbf{e}_{p}\right|$ vs. time (forward-looking camera).
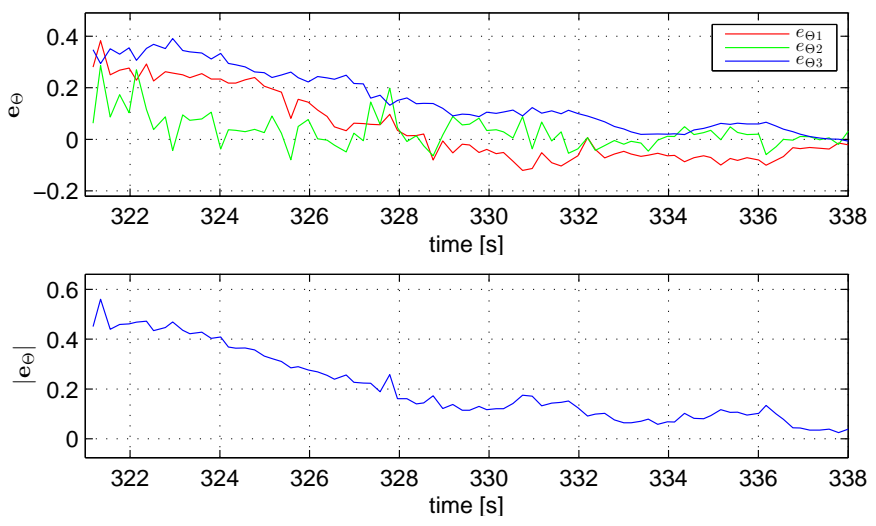

Fig. 17: $\mathbf{e}_{\Theta}$ and $\left|\mathbf{e}_{\Theta}\right|$ vs. time (forward-looking camera).

AUVs using image-based homography matrix. The originality of the proposed approach lies in exploiting the full system dynamics in control design while the knowledge of the relative depth, normal vector, and size of the observed scene are not required. The controller ensures almost global asymptotic stability as well as robustness with respect to unmodeled dynamics. Rigorous stability analysis for closed-loop systems has been given. Simulations provide a clear picture of the predicted response of the proposed algorithm, showing clearly an improved performance w.r.t. the state-of-the-art HBVS control [5]. The experimental results show that the proposed scheme is effective, even when the system parameters are not known precisely. As perspectives, several directions are of interest. Some practical situations limit the applications of the proposed approach to the stabilisation tasks. Exploiting other image features and adapting the outer-loop to these new features to perform other tasks such as pipe following, docking, etc. would already provide a major improvement in practical inspection or monitoring scenario. How to carry out image-based stabilisation task by underactuated AUVs is challenging and would be addressed as direct extension of the proposed work.

\section{APPENDIX A \\ PROOF OF LEMMA 2}

In first-order approximations, $\mathbf{R} \approx \mathbf{I}_{3}+\boldsymbol{\Theta}_{\times}$, with $\Theta \in \mathbb{R}^{3}$. One then verifies from 8 that in first-order approximations

$$
\mathbf{e}_{p} \approx a^{\star} \mathbf{p}_{C}-\mathbf{m}_{\times}^{\star} \boldsymbol{\Theta}, \quad \mathbf{e}_{\Theta} \approx \frac{1}{d^{\star}} \mathbf{n}_{\times}^{\star} \mathbf{p}_{C}+2 \boldsymbol{\Theta},
$$




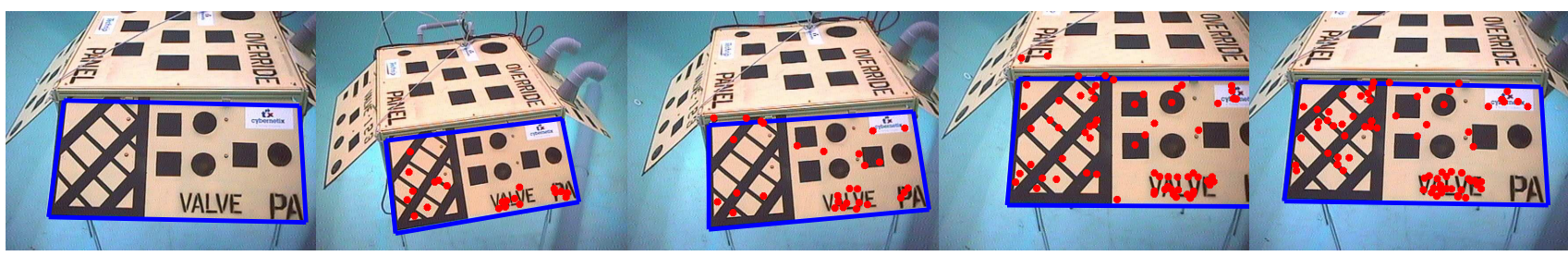

(a) Reference image

(b) $\mathrm{t}=321 \mathrm{~s}$

(c) $t=326 \mathrm{~s}$

(d) $\mathrm{t}=331 \mathrm{~s}$

(e) $t=336 \mathrm{~s}$

Fig. 18: Reference image and current images with forward-looking camera.

with $a^{\star} \triangleq \frac{\left(\mathbf{n}^{\star \top} \mathbf{m}^{\star}\right)}{d^{\star}}$. Using the first-order approximations $\dot{\boldsymbol{\Theta}} \approx$ $\boldsymbol{\Omega}$ and $\dot{\mathbf{p}}_{C} \approx \mathbf{V}_{C}$, one verifies that the linearised system is $\dot{\mathbf{X}}=\mathbf{A X}$, with $\mathbf{X} \in \mathbb{R}^{12}$ and $\mathbf{A} \in \mathbb{R}^{12 \times 12}$ given by

$$
\begin{aligned}
& \mathbf{A} \triangleq\left[\begin{array}{cccc}
-a^{\star} k_{p} \mathbf{I}_{3} & k_{\Theta} \mathbf{m}_{\times}^{\star} & a^{\star} \mathbf{I}_{3} & -\mathbf{m}_{\times}^{\star} \\
-\frac{k_{p}}{d^{\star}} \mathbf{n}_{\times}^{\star} & -2 k_{\Theta} \mathbf{I}_{3} & \frac{1}{d^{\star}} \mathbf{n}_{\times}^{\star} & 2 \mathbf{I}_{3} \\
-a^{\star} k_{p}^{2} \mathbf{I}_{3} & k_{p} k_{\Theta} \mathbf{m}_{\times}^{\star} & \left(a^{\star} k_{p}-k_{V}\right) \mathbf{I}_{3} & -k_{p} \mathbf{m}_{\times}^{\star} \\
-\frac{k_{p} k_{\Theta}}{d^{\star}} \mathbf{n}_{\times}^{\star} & -2 k_{\Theta}^{2} \mathbf{I}_{3} & \frac{k_{\Theta}}{d^{\star}} \mathbf{n}_{\times}^{\star} & \left(2 k_{\Theta}-k_{\Omega}\right) \mathbf{I}_{3}
\end{array}\right], \\
& \mathbf{X} \triangleq\left[\begin{array}{llll}
\mathbf{e}_{p}^{\top} & \mathbf{e}_{\Theta}^{\top} & \tilde{\mathbf{V}}_{C}^{\top} & \widetilde{\boldsymbol{\Omega}}^{\top}
\end{array}\right]^{\top}, \\
& \widetilde{\mathbf{V}}_{C} \triangleq \mathbf{V}_{C}-\mathbf{V}_{C r}, \quad \widetilde{\boldsymbol{\Omega}} \triangleq \boldsymbol{\Omega}-\boldsymbol{\Omega}_{r} .
\end{aligned}
$$

After long and tedious computations, we have verified that the $12^{\text {th }}$-order characteristic polynomial of this linearised system is given by $P(\lambda)=P_{1}(\lambda) P_{2}(\lambda)\left(P_{3}(\lambda)\right)^{2}$, with

$$
\begin{aligned}
P_{1}(\lambda) \triangleq & \lambda^{2}+k_{V} \lambda+a^{\star} k_{p} k_{V} \\
P_{2}(\lambda) \triangleq & \lambda^{2}+k_{\Omega} \lambda+2 k_{\Theta} k_{\Omega} \\
P_{3}(\lambda) \triangleq & \lambda^{4}+\left(k_{V}+k_{\Omega}\right) \lambda^{3}+\left(a^{\star} k_{p} k_{V}+2 k_{\Theta} k_{\Omega}+k_{V} k_{\Omega}\right) \lambda^{2} \\
& +\left(a^{\star} k_{p}+2 k_{\Theta}\right) k_{V} k_{\Omega} \lambda+a^{\star} k_{p} k_{\Theta} k_{V} k_{\Omega}
\end{aligned}
$$

which implies the stability of the origin of the linearised system by direct application of Routh-Hurwitz criterion.

\section{APPENDIX B \\ PROOF OF LEMMA 3}

Using (7) and (8), one obtains

$$
\dot{\mathbf{e}}_{p}=-\boldsymbol{\Omega} \times\left(\mathbf{e}_{p}-m^{\star}\right)+a^{\star} \mathbf{V}_{C} .
$$

Consider $\boldsymbol{\Omega} \equiv 0$ and $\widetilde{\mathbf{V}}_{C} \triangleq \mathbf{V}_{C}-\mathbf{V}_{C r}$ with $\mathbf{V}_{C r}=-k_{p} \mathbf{e}_{p}$, it suffices to prove there exists some positive gains $k_{p}, k_{V}$ and $k_{i V}$ for which the previous system can be unstable. One easily verifies that the closed loop control system can be written as $\dot{\mathbf{X}}=\mathbf{A X}$, with

$$
\mathbf{A} \triangleq\left[\begin{array}{ccc}
-a^{\star} k_{p} & 0 & a^{\star} \\
0 & 0 & 1 \\
-a^{\star} k_{p}^{2} & -k_{i V} & a^{\star} k_{p}-k_{V}
\end{array}\right],
$$

and $\mathbf{X} \triangleq\left[\begin{array}{ccc}\mathbf{e}_{p}^{\top} & \left(\int \widetilde{\mathbf{V}}_{C}\right)^{\top} & \widetilde{\mathbf{V}}_{C}^{\top}\end{array}\right]^{\top}$. From here, a simple application of Routh-Hurwitz criterion ensures that if condition (15) is satisfied, then the linearized system is unstable.

\section{APPENDiX C}

\section{PROOF OF LEMMA 4}

Using (16), (17) and (18), one verifies that

$$
\dot{\mathbf{e}}_{p}=-\boldsymbol{\Omega} \times \mathbf{e}_{p}-a^{\star} k_{p} \mathbf{e}_{p}-a^{\star}\left(z_{p}-1 / a^{\star}\right) \boldsymbol{\Omega} \times \mathbf{m}^{\star} .
$$

Consider the following candidate Lyapunov function

$$
\mathcal{L}_{0} \triangleq \frac{1}{2 a^{\star}}\left|\mathbf{e}_{p}\right|^{2}+\frac{1}{2}\left|z_{p}-1 / a^{\star}\right|^{2} .
$$

Using (17) and (36), one verifies that the derivative of $\mathcal{L}_{0}$ satisfies $\mathcal{L}_{0}=-k_{p}\left|\mathbf{e}_{p}\right|^{2}$. From here, one ensures that $\mathcal{L}_{0}$ and, thus, $\mathbf{e}_{p}$ and $z_{p}$ are bounded w.r.t. initial conditions. The resulting boundedness of $\dot{\mathbf{e}}_{p}$ given in $(36)$ and, thus, of $\ddot{\mathcal{L}}_{0}$ implies the uniform continuity of $\dot{\mathcal{L}}_{0}$. Finally, the application of Barbalat's lemma [19] ensures the convergence of $\dot{\mathcal{L}}_{0}$ to zero. This concludes the proof.

\section{APPENDIX D}

PROOF OF PROPOSITION 1

The proof proceeds by three steps:

Step 1: We will show that $z_{p}$ is bounded by some constant. Consider the positive function $\mathcal{S}_{1} \triangleq 0.5 z_{p}^{2}$. Its derivative satisfies (using (19)) $\dot{\mathcal{S}}_{1} \leq-k_{z} \nabla z_{p}^{2}+\left|z_{p}\right| \nabla\left(\bar{\Omega}_{r}+k_{z} \Delta\right.$ ), with $\bar{\Omega}_{r} \triangleq \sup \left(\left|\boldsymbol{\Omega}_{r}\right|\right)$. From here, it is straightforward to deduce that $\left|z_{p}(t)\right| \leq \alpha_{r} \triangleq k_{z}^{-1} \bar{\Omega}_{r}+\Delta, \forall t \geq 0$.

Step 2: We will show next that there exists a time instant $T$ such that $\forall \tau \geq T$ one has $\left|\mathbf{e}_{p}(\tau)\right| \leq \nabla$ and, thus, $\operatorname{sat}^{\nabla}\left(\mathbf{e}_{p}(\tau)\right)=\mathbf{e}_{p}(\tau)$.

Using (16) and (20), one deduces

$$
\dot{\mathbf{e}}_{p}=-\boldsymbol{\Omega} \times \mathbf{e}_{p}-a^{\star} \bar{z}_{p} \boldsymbol{\Omega}_{r} \times \mathbf{m}^{\star}-a^{\star} k_{p 1} \hat{\mathbf{e}}_{p}+\gamma\left(\widetilde{\mathbf{V}}_{C}, \widetilde{\boldsymbol{\Omega}}\right),
$$

with $\gamma\left(\widetilde{\mathbf{V}}_{C}, \widetilde{\boldsymbol{\Omega}}\right) \triangleq a^{\star} \widetilde{\mathbf{V}}_{C}+\widetilde{\boldsymbol{\Omega}} \times \mathbf{m}^{\star}, \bar{z}_{p} \triangleq z_{p}-\frac{1}{a^{\star}}$. Denote $\mathbf{X} \triangleq[\mathbf{x}, \mathbf{y}]^{\top} \in \mathbb{R}^{6}$, with $\mathbf{x} \triangleq \mathbf{R}_{\hat{e}_{p}}, \mathbf{y} \triangleq \mathbf{R e}_{p}$. One verifies from (19) and 37) that

$$
\dot{\mathbf{X}}=\underbrace{\left[\begin{array}{cc}
-k_{p 2} \mathbf{I}_{3} & k_{p 2} \mathbf{I}_{3} \\
-a^{\star} k_{p 1} \mathbf{I}_{3} & \mathbf{0}
\end{array}\right]}_{\triangleq \mathbf{A} \in \mathbb{R}^{6 \times 6}} \mathbf{X}+\underbrace{\left[\begin{array}{c}
\mathbf{0} \\
\mathbf{R}\left(-a^{\star} \bar{z}_{p} \boldsymbol{\Omega}_{r \times} \mathbf{m}^{\star}+\gamma\left(\widetilde{\mathbf{V}}_{C}, \widetilde{\boldsymbol{\Omega}}\right)\right)
\end{array}\right]}_{\triangleq \mathbf{B} \in \mathbb{R}^{6}}
$$

If $k_{p 2} / k_{p_{1}}>\bar{\kappa}_{p} \triangleq 4 a^{\star}$, then $\mathbf{A}$ has two triple distinct real negative eigenvalues $\lambda_{1,2}\left(\lambda_{1}<\lambda_{2}<0\right)$ given by $\lambda_{1,2}=0.5\left(-k_{p 2} \mp \sqrt{k_{p 2}^{2}-4 a^{\star} k_{p 1} k_{p 2}}\right)$. This implies that $\mathbf{A}$ is diagonalisable and can be decomposed in the Jordan normal form $\mathbf{A}=\mathbf{P} \boldsymbol{\Lambda} \mathbf{P}^{-1}$, with $\boldsymbol{\Lambda}=\operatorname{diag}\left(\lambda_{1} \mathbf{I}_{3}, \lambda_{2} \mathbf{I}_{3}\right)$ and

$$
\begin{gathered}
\mathbf{P}=\left[\begin{array}{cc}
\frac{\lambda_{1} \mathbf{I}_{3}}{\eta_{1}} & \frac{\lambda_{2} \mathbf{I}_{3}}{\eta_{2}} \\
\frac{-a^{\star} k_{p 1} \mathbf{I}_{3}}{\eta_{1}} & \frac{-a^{\star} k_{p 1} \mathbf{I}_{3}}{\eta_{2}}
\end{array}\right], \mathbf{P}^{-1}=\frac{1}{\lambda_{2}-\lambda_{1}}\left[\begin{array}{cc}
-\eta_{1} \mathbf{I}_{3} & \frac{-\lambda_{2} \eta_{1} \mathbf{I}_{3}}{\star^{\star} k_{p 1}} \\
\eta_{2} \mathbf{I}_{3} & \frac{\lambda_{1} \eta_{2} \mathbf{I}_{3}}{a^{\star} k_{p 1}}
\end{array}\right] \\
\eta_{1} \triangleq \sqrt{\lambda_{1}^{2}+\left(a^{\star} k_{p 1}\right)^{2}}, \quad \eta_{2} \triangleq \sqrt{\lambda_{2}^{2}+\left(a^{\star} k_{p 1}\right)^{2}} .
\end{gathered}
$$

By simple calculations, it can be verified that

$$
\begin{aligned}
& \left(\lambda_{2}-\lambda_{1}\right)^{2}\left|\mathbf{P}^{-1} \mathbf{X}\right|^{2}=\eta_{1}^{2}\left|\mathbf{x}+\frac{\lambda_{2}}{\left(a^{\star} k_{p 1}\right)} \mathbf{y}\right|^{2}+\eta_{2}^{2}\left|\mathbf{x}+\frac{\lambda_{1} \mathbf{y}}{a^{\star} k_{p 1}}\right|^{2} \\
& =\left|\sqrt{\eta_{1}^{2}+\eta_{2}^{2}}\left(\mathbf{x}+\frac{\lambda_{1} \mathbf{y}}{a^{\star} k_{p 1}}\right)+\frac{\eta_{1}^{2}\left(\lambda_{2}-\lambda_{1}\right) \mathbf{y}}{a^{\star} k_{p 1} \sqrt{\eta_{1}^{2}+\eta_{2}^{2}}}\right|^{2}+\frac{\eta_{1}^{2} \eta_{2}^{2}\left(\lambda_{2}-\lambda_{1}\right)^{2}|\mathbf{y}|^{2}}{\left(a^{\star} k_{p 1}\right)^{2}\left(\eta_{1}^{2}+\eta_{2}^{2}\right)},
\end{aligned}
$$

which allows one to deduce

$$
\left|\mathbf{P}^{-1} \mathbf{X}\right| \geq \frac{\eta_{1} \eta_{2}}{a^{\star} k_{p 1} \sqrt{\eta_{1}^{2}+\eta_{2}^{2}}}|\mathbf{y}|=\frac{\eta_{1} \eta_{2}}{a^{\star} k_{p 1} \sqrt{\eta_{1}^{2}+\eta_{2}^{2}}}\left|\mathbf{e}_{p}\right| .
$$

On the other hand, since the inner loop controller ensures that $\widetilde{\boldsymbol{\Omega}}$ and $\widetilde{\mathbf{V}}$ converge asymptotically to zero, $\gamma\left(\widetilde{\mathbf{V}}_{C}, \widetilde{\boldsymbol{\Omega}}\right)$ also converges to zero. Thus, for some positive number $\varepsilon$ (to be specified hereafter) there exists a time instant $T_{1}$ such that 
$\forall t \geq T_{1}$ one has $\left|\gamma\left(\widetilde{\mathbf{V}}_{C}, \widetilde{\Omega}\right)\right|<\varepsilon$. Then, $\forall t \geq T_{1}$ one verifies

$$
\begin{aligned}
\left|\mathbf{P}^{-1} \mathbf{B}\right| & =\frac{\sqrt{\lambda_{1}^{2} \eta_{2}^{2}+\lambda_{2}^{2} \eta_{1}^{2}}}{a^{\star} k_{p 1}\left(\lambda_{2}-\lambda_{1}\right)}\left|-a^{\star} \bar{z}_{p} \boldsymbol{\Omega}_{r} \times \overline{\mathbf{m}}^{\star}+\gamma\left(\widetilde{\mathbf{V}}_{C}, \widetilde{\boldsymbol{\Omega}}\right)\right| \\
& <\frac{\sqrt{\lambda_{1}^{2} \eta_{2}^{2}+\lambda_{2}^{2} \eta_{1}^{2}}}{a^{\star} k_{p 1}\left(\lambda_{2}-\lambda_{1}\right)}\left(\left(a^{\star} \alpha_{r}+1\right) \bar{\Omega}_{r}+\varepsilon\right) .
\end{aligned}
$$

Using [38), the derivative of $\mathcal{S}_{2} \triangleq 0.5\left|\mathbf{P}^{-1} \mathbf{X}\right|^{2}$ satisfies

$$
\begin{aligned}
\dot{\mathcal{S}}_{2} & =\left(\mathbf{P}^{-1} \mathbf{X}\right)^{\top} \boldsymbol{\Lambda}\left(\mathbf{P}^{-1} \mathbf{X}\right)+\left(\mathbf{P}^{-1} \mathbf{X}\right)^{\top}\left(\mathbf{P}^{-1} \mathbf{B}\right) \\
& \leq \lambda_{2}\left|\mathbf{P}^{-1} \mathbf{X}\right|^{2}+\left|\mathbf{P}^{-1} \mathbf{X}\right|\left|\mathbf{P}^{-1} \mathbf{B}\right| .
\end{aligned}
$$

Since $z_{p}, \boldsymbol{\Omega}_{r}, \widetilde{\boldsymbol{\Omega}}$ and $\widetilde{\mathbf{V}}_{C}$ are bounded, $\mathbf{B}$ and $\mathbf{P}^{-1} \mathbf{B}$ are also bounded. From (41) and the definition of $\mathcal{S}_{2}$, one ensures that $\mathbf{P}^{-1} \mathbf{X}$ and $\mathbf{X}$ are bounded w.r.t. initial conditions. Consequently, $\mathbf{e}_{p}$ and $\hat{\mathbf{e}}_{p}$ remain bounded w.r.t. initial conditions. Then, it is straightforward to verify that $\dot{\mathbf{e}}_{p}$, $\dot{\hat{\mathbf{e}}}_{p}$ and $\dot{z}_{p}$ are bounded w.r.t. initial conditions, which implies the uniform continuity of $\mathbf{e}_{p}, \hat{\mathbf{e}}_{p}$ and $z_{p}$.

Since $\mathbf{X}$ remains bounded w.r.t. initial conditions on the time-interval $\left[0, T_{1}\right]$ (as proved previously), from (41) and the definition of $\mathcal{S}_{2}$ there exists another time-instant $T>T_{1}$ such that $\forall \tau \geq T$ one has

$$
\left|\mathbf{P}^{-1} \mathbf{X}(\tau)\right| \leq-\lambda_{2}^{-1}\left(\sup _{t \geq T}\left(\left|\mathbf{P}^{-1} \mathbf{B}(t)\right|\right)+\bar{\varepsilon}\right),
$$

with $0<\bar{\varepsilon} \leq \frac{\sqrt{\lambda_{1}^{2} \eta_{2}^{2}+\lambda_{2}^{2} \eta_{1}^{2}}}{a^{\star} k_{p 1}\left(\lambda_{2}-\lambda_{1}\right)} \varepsilon$. From [39), 440) and 442) one deduces that

$$
\left|\mathbf{e}_{p}(\tau)\right| \leq \bar{\nabla}+\varepsilon \frac{2 \sqrt{\left(\eta_{1}^{2}+\eta_{2}^{2}\right)\left(\lambda_{1}^{2} \eta_{2}^{2}+\lambda_{2}^{2} \eta_{1}^{2}\right)}}{\lambda_{2}\left(\lambda_{1}-\lambda_{2}\right) \eta_{1} \eta_{2}}, \forall \tau \geq T
$$

with $\bar{\nabla} \triangleq \frac{\sqrt{\left(\eta_{1}^{2}+\eta_{2}^{2}\right)\left(\lambda_{1}^{2} \eta_{2}^{2}+\lambda_{2}^{2} \eta_{1}^{2}\right)}}{\lambda_{2}\left(\lambda_{1}-\lambda_{2}\right) \eta_{1} \eta_{2}}\left(a^{\star} \alpha_{r}+1\right) \bar{\Omega}_{r}>0$. Therefore, if $\nabla$ is chosen larger than $\bar{\nabla}$ (i.e., $\nabla>\bar{\nabla}$ ) and if $\varepsilon$ is chosen such that

$$
0<\varepsilon<\frac{(\nabla-\bar{\nabla}) \lambda_{2}\left(\lambda_{1}-\lambda_{2}\right) \eta_{1} \eta_{2}}{2 \sqrt{\left(\eta_{1}^{2}+\eta_{2}^{2}\right)\left(\lambda_{1}^{2} \eta_{2}^{2}+\lambda_{2}^{2} \eta_{1}^{2}\right)}}
$$

then one deduces from inequality (43) that $\left|\mathbf{e}_{p}(\tau)\right|<\nabla, \forall \tau \geq$ $T$, and thus $\operatorname{sat}^{\nabla}\left(\mathbf{e}_{p}(\tau)\right)=\mathbf{e}_{p}(\tau)$.

Step 3: Consider the candidate Lyapunov function

$$
\mathcal{L} \triangleq \frac{1}{2 a^{\star}}\left|\mathbf{e}_{p}\right|^{2}+\frac{k_{p 1}}{2 k_{p 2}}\left|\hat{\mathbf{e}}_{p}\right|^{2}+\frac{1}{2}\left|\bar{z}_{p}\right|^{2} .
$$

Using the following property [16]

$$
\left|\operatorname{sat}^{\Delta}(\mathbf{x}+\mathbf{c})-\mathbf{c}\right| \leq|\mathbf{x}|, \forall(\mathbf{c}, \mathbf{x}) \in \mathbb{R}^{3} \times \mathbb{R}^{3} \text { with }|\mathbf{c}| \leq \Delta,
$$

one deduces

$$
\begin{aligned}
\dot{\mathcal{L}}= & -k_{1}\left|\hat{\mathbf{e}}_{p}\right|^{2}-\bar{z}_{p}\left(\mathbf{e}_{p}-\operatorname{sat}^{\nabla}\left(\mathbf{e}_{p}\right)\right)^{\top} \boldsymbol{\Omega}_{r \times} \mathbf{m}^{\star} \\
& \quad-k_{z} \nabla \bar{z}_{p}^{\top}\left(\bar{z}_{p}+\frac{1}{a^{\star}}-\operatorname{sat}^{\Delta}\left(\bar{z}_{p}+\frac{1}{a^{\star}}\right)\right)+\frac{1}{a^{\star}} \mathbf{e}_{p}^{\top} \gamma\left(\widetilde{\mathbf{V}}_{C}, \widetilde{\boldsymbol{\Omega}}^{\prime}\right) \\
\leq & -k_{1}\left|\hat{\mathbf{e}}_{p}\right|^{2}-\bar{z}_{p}\left(\mathbf{e}_{p}-\operatorname{sat}^{\nabla}\left(\mathbf{e}_{p}\right)\right)^{\top} \boldsymbol{\Omega}_{r \times} \mathbf{m}^{\star}+\frac{1}{a^{\star}} \mathbf{e}_{p}^{\top} \gamma\left(\widetilde{\mathbf{V}}_{C}, \widetilde{\boldsymbol{\Omega}}\right) .
\end{aligned}
$$

Since $\operatorname{sat}^{\nabla}\left(\mathbf{e}_{p}(\tau)\right)=\mathbf{e}_{p}(\tau)$ and $\left|\mathbf{e}_{p}(\tau)\right| \leq \nabla, \forall \tau \geq T$ (as proved in Step 2), one obtains

$$
\dot{\mathcal{L}}(\tau) \leq-k_{1}\left|\hat{\mathbf{e}}_{p}(\tau)\right|^{2}+\frac{\nabla}{a^{\star}}\left|\gamma\left(\widetilde{\mathbf{V}}_{C}(\tau), \widetilde{\boldsymbol{\Omega}}(\tau)\right)\right| .
$$

As proved previously, $\mathbf{e}_{p}, \hat{\mathbf{e}}_{p}$ and $z_{p}$ cannot escape in finitetime. Thus, $\mathcal{L}(t)$ remains bounded on the time-interval $[0, T]$. In addition, $\mathbf{e}_{p}(\tau), \hat{\mathbf{e}}_{p}(\tau), z_{p}(\tau)$ and, thus, $\mathcal{L}(\tau), \forall \tau \geq T$, remain bounded as proved previously. Since the equilibrium $\left(\widetilde{\mathbf{V}}_{C}, \widetilde{\boldsymbol{\Omega}}\right)=(\mathbf{0}, \mathbf{0})$ is locally exponentially stable as a result of the inner-loop controller, there exist some time-instant $T_{2}>T$ and some positive constants $\alpha_{1}$ and $\alpha_{2}$ such that

$$
\left|\gamma\left(\widetilde{\mathbf{V}}_{C}(\tau), \widetilde{\boldsymbol{\Omega}}(\tau)\right)\right| \leq \alpha_{1} e^{-\alpha_{2} \tau}, \quad \forall \tau \geq T_{2} .
$$

From (44) and (45), one deduces

$$
\dot{\mathcal{L}}(\tau) \leq-k_{1}\left|\hat{\mathbf{e}}_{p}(\tau)\right|^{2}+\frac{\alpha_{1} \nabla}{a^{\star}} e^{-\alpha_{2} \tau}, \quad \forall \tau \geq T_{2} .
$$

Consequently, by integration one deduces

$$
\int_{T_{2}}^{\infty}\left|\hat{\mathbf{e}}_{p}(\tau)\right|^{2} d \tau \leq \frac{\alpha_{1} \nabla e^{-\alpha_{2} T_{2}}}{k_{1} \alpha_{2} a^{\star}}+\frac{1}{k_{1}}\left(\mathcal{L}\left(T_{2}\right)-\mathcal{L}(\infty)\right) .
$$

From here, the resulting boundedness of integral term $\int_{T_{2}}^{\infty}\left|\hat{\mathbf{e}}_{p}(\tau)\right|^{2} d \tau$ and the uniform continuity of $\hat{\mathbf{e}}_{p}$ implies the convergence of $\hat{\mathbf{e}}_{p}$ to zero (Barbalat's lemma).

From (19), one verifies that $\dot{\hat{\mathbf{e}}}_{p}$ can be rewritten as $\dot{\hat{\mathbf{e}}}_{p}(t)=$ $\mathbf{a}(t)+\mathbf{b}(t)$, with $\mathbf{a}(t) \triangleq k_{2} \mathbf{e}_{p}$ the uniformly continuous term and $\mathbf{b}(t) \triangleq-\boldsymbol{\Omega}_{\times} \hat{\mathbf{e}}_{p}-k_{2} \hat{\mathbf{e}}_{p}$ the vanishing term. Then, the application of the extended Barbalat's lemma [29] ensures the convergence of $\dot{\hat{\mathbf{e}}}_{p}$ to zero, which in turn implies the convergence of $\mathbf{e}_{p}$ to zero.

\section{APPENDIX E \\ PROOF OF PROPOSITION 2}

Property 1 of Proposition 2 can be directly deduced from the dynamics 21) of $\boldsymbol{\Omega}_{r}$. Now let us prove that the equilibrium $\left(\mathbf{H}, \mathbf{V}_{C}, \boldsymbol{\Omega}\right)=\left(\mathbf{I}_{3}, \mathbf{0}, \mathbf{0}\right)$ is LAS. Using the approximations of $\mathbf{e}_{p}$ and $\mathbf{e}_{\Theta}$ given by (34) one deduces that

$$
\mathbf{e}_{\Theta} \approx\left(2 \mathbf{I}_{3}+\frac{1}{\gamma^{\star}} \mathbf{n}_{\times}^{\star} \mathbf{m}_{\times}^{\star}\right) \Theta+\frac{1}{\gamma^{\star}} \mathbf{n}_{\times}^{\star} \mathbf{e}_{p},
$$

with $\gamma^{\star} \triangleq \mathbf{n}^{\star \top} \mathbf{m}^{\star}$. Using also the approximation $\dot{\boldsymbol{\Theta}} \approx \boldsymbol{\Omega}$, one obtains the following linearised dynamics $\dot{\mathbf{X}}_{1}=\mathbf{A}_{1} \mathbf{X}_{1}+$ $\mathbf{G}_{1}\left(\widetilde{\mathbf{\Omega}}, \mathbf{e}_{p}\right)$, with $\mathbf{X}_{1} \triangleq\left[\begin{array}{ll}\dot{\Theta}^{\top} & \dot{\boldsymbol{\Omega}}_{r}^{\top}\end{array}\right]^{\top}$,

$$
\begin{aligned}
& \mathbf{A}_{1} \triangleq\left[\begin{array}{cc}
\mathbf{0} & \mathbf{I}_{3} \\
-k_{\Theta 1}\left(2 \mathbf{I}_{3}+\frac{1}{\gamma^{\star}} \mathbf{n}_{\times}^{\star} \mathbf{m}_{\times}^{\star}\right) & -k_{\Theta 2} \mathbf{I}_{3}
\end{array}\right], \\
& \mathbf{G}_{1}\left(\widetilde{\boldsymbol{\Omega}}, \mathbf{e}_{p}\right) \triangleq\left[\widetilde{\boldsymbol{\Omega}}^{\top} \quad \frac{k_{\Theta 1}}{\gamma^{\star}} \mathbf{n}_{\times}^{\star} \mathbf{e}_{p}^{\top}\right]^{\top} .
\end{aligned}
$$

The perturbation term $\mathbf{G}_{1}\left(\widetilde{\boldsymbol{\Omega}}, \mathbf{e}_{p}\right)$ converges asymptotically to zero since $\widetilde{\boldsymbol{\Omega}}$ and $\mathbf{e}_{p}$ converge to zero as a consequence of the inner-loop controller and Proposition 1 Then, it suffices to prove the exponential stability of the nominal linear system $\dot{\mathbf{X}}_{1}=\mathbf{A}_{1} \mathbf{X}_{1}$, whose characteristic polynomial is given by

$$
P_{1}(\lambda)=\left(\lambda^{2}+k_{\Theta 2} \lambda+2 k_{\Theta 1}\right)\left(\lambda^{2}+k_{\Theta 2} \lambda+k_{\Theta 1}\right) .
$$

This nominal system is, indeed, stable by direct application of Routh-Hurwitz criterion. We have proved the local convergence of $\mathbf{e}_{p}$ and $\mathbf{e}_{\Theta}$ to zero, which implies that the equilibrium $\left(\mathbf{H}, \mathbf{V}_{C}, \boldsymbol{\Omega}\right)=\left(\mathbf{I}_{3}, \mathbf{0}, \mathbf{0}\right)$ is LAS. The local exponential stability proof of this equilibrium is more involved. In fact, the linearised dynamics of $\mathbf{X}_{2} \triangleq\left[\begin{array}{llll}\Theta^{\top} & \boldsymbol{\Omega}_{r}^{\top} & \hat{\mathbf{e}}_{p}^{\top} & \mathbf{e}_{p}^{\top}\end{array}\right]^{\top}$ are given by $\dot{\mathbf{X}}_{2}=\mathbf{A}_{2} \mathbf{X}_{2}+\mathbf{G}_{2}\left(\widetilde{\mathbf{V}}_{C}, \widetilde{\mathbf{\Omega}}\right)$, with

$$
\begin{gathered}
\mathbf{A}_{2} \triangleq\left[\begin{array}{cccc}
\mathbf{0} & \mathbf{I}_{3} & \mathbf{0} & \mathbf{0} \\
-k_{\Theta 1}\left(2 \mathbf{I}_{3}+\frac{1}{\gamma^{\star}} \mathbf{n}_{\times}^{\star} \mathbf{m}_{\times}^{\star}\right) & -k_{\Theta 2} \mathbf{I}_{3} & \mathbf{0} & -\frac{k_{\Theta 1}}{\gamma^{\star}} \mathbf{n}_{\times}^{\star} \\
\mathbf{0} & \mathbf{0} & -k_{p 2} \mathbf{I}_{3} & k_{p 2} \mathbf{I}_{3} \\
\mathbf{0} & \left(-1+a^{\star} z_{p}\right) \mathbf{m}_{\times}^{\star} & -a^{\star} k_{p 1} \mathbf{I}_{3} & \mathbf{0}
\end{array}\right] \\
\mathbf{G}_{2}\left(\widetilde{\mathbf{V}}_{C}, \widetilde{\boldsymbol{\Omega}}\right) \triangleq\left[\begin{array}{lllll}
\widetilde{\boldsymbol{\Omega}}^{\top} & \mathbf{0}^{\top} & \mathbf{0}^{\top} & a^{\star} \widetilde{\mathbf{V}}_{C}
\end{array}\right]^{\top} .
\end{gathered}
$$

The perturbation term $\mathbf{G}_{2}\left(\widetilde{\mathbf{V}}_{C}, \widetilde{\boldsymbol{\Omega}}\right)$ converges to exponentially zero as a result of the inner-loop controller. Thus, it suffices to prove the exponential stability of the nominal system $\dot{\mathbf{X}}_{2}=$ $\mathbf{A}_{2} \mathbf{X}_{2}$. One verifies that its characteristic polynomial is $P_{2}(\lambda)=\left(\lambda^{2}+k_{\Theta_{2}} \lambda+2 k_{\Theta 1}\right)\left[\lambda^{4}+a_{3} \lambda^{3}+a_{2} \lambda^{2}+a_{1} \lambda+a_{0}\right]^{2}$, 


$$
\left\{\begin{array}{l}
a_{3} \triangleq k_{p 2}+k_{\Theta 2} \\
a_{2} \triangleq a^{\star} k_{p 1} k_{p 2}+k_{p 2} k_{\Theta 2}+\left(2-a^{\star} z_{p}\right) k_{\Theta 1} \\
a_{1} \triangleq a^{\star} k_{p 1} k_{p 2} k_{\Theta 2}+\left(2-a^{\star} z_{p}\right) k_{p 1} k_{\Theta 1} \\
a_{0} \triangleq a^{\star} k_{p 1} k_{p 2} k_{\Theta 1}
\end{array}\right.
$$

By application of Routh-Hurwitz criterion, the nominal system $\dot{\mathbf{X}}_{2}=\mathbf{A}_{2} \mathbf{X}_{2}$ is exponentially stable if

$a_{0,1,2,3}>0, b_{1} \triangleq \frac{a_{3} a_{2}-a_{1}}{a_{3}}>0, c_{1} \triangleq \frac{b_{1} a_{1}-a_{3} a_{0}}{b_{1}}>0$, or, equivalently, if the following conditions are satisfied:

$$
\begin{array}{ll}
a^{\star} k_{p 1} k_{p 2}+k_{p 2} k_{\Theta 2}+\left(1+\gamma_{z}\right) k_{\Theta 1}>0 & \text { (46a) } \\
a^{\star} k_{p 1} k_{p 2} k_{\Theta 2}+\left(1+\gamma_{z}\right) k_{p 1} k_{\Theta 1}>0 & \text { (46b) } \\
a^{\star} k_{p 1} k_{p 2}^{2}+k_{p 2} k_{\Theta 2}\left(k_{p 2}+k_{\Theta 2}\right)+\left(1+\gamma_{z}\right) k_{\Theta 1} k_{\Theta 2}>0 & (46 \mathrm{c}) \\
\left(a^{\star} k_{p 1} k_{p 2}-k_{\Theta 1}\right)^{2} k_{\Theta 2}+k_{p 2} k_{\Theta 2}\left(k_{p 2}+k_{\Theta 2}\right)\left(a^{\star} k_{p 1} k_{\Theta 2}+k_{\Theta 1}\right) \\
+\gamma_{z}\left(k_{p 1} k_{p 2}^{2}+k_{p 1} k_{\Theta 2}^{2}+2 k_{\Theta 1} k_{\Theta 2}+k_{p 2} k_{\Theta 2}\left(k_{p 2}+k_{\Theta 2}\right)\right) k_{\Theta 1} \\
+\gamma_{z}^{2} k_{\Theta 1}^{2} k_{\Theta 2}>0
\end{array}
$$

with $\gamma_{z} \triangleq 1-a^{\star} z_{p}$. Since $z_{p}$, and thus, $\gamma_{z}$ are bounded as a result of Proposition 1, it is obvious that by making $k_{\Theta 1}$ tending to zero all conditions in (46) hold. Therefore, there exists a positive number $\underline{k}_{\Theta 1}$, depending on $\left(k_{p 1}, k_{p 2}, k_{\Theta 1}, k_{\Theta 2}, a^{\star}, z_{p}\right)$, such that for all $k_{\Theta 1}<\underline{k}_{\Theta 1}$ all conditions in (46) are satisfied. This allows one to conclude the proof.

\section{APPENDIX F \\ PROOF OF THEOREM 1}

We first make the following changes of variables:

$\overline{\mathbf{R}} \triangleq \mathbf{R}_{u} \mathbf{R R}_{u}^{\top}, \overline{\mathbf{p}}_{C} \triangleq \mathbf{R}_{u} \mathbf{p}_{C}, \overline{\mathbf{n}}^{\star} \triangleq \mathbf{R}_{u} \mathbf{n}^{\star}, \overline{\mathbf{m}}^{\star} \triangleq \mathbf{R}_{u} \mathbf{m}^{\star}$,

$\overline{\mathbf{H}} \triangleq \overline{\mathbf{R}}^{\top}-\frac{1}{d^{\star}} \overline{\mathbf{R}}^{\top} \overline{\mathbf{p}}_{C} \overline{\mathbf{n}}^{\star \top}, \overline{\boldsymbol{\Omega}} \triangleq \mathbf{R}_{u} \boldsymbol{\Omega}$,

$\overline{\mathbf{e}}_{p} \triangleq \mathbf{R}_{u} \mathbf{e}_{p}$

from which one easily verifies that $\overline{\mathbf{H}}=\mathbf{R}_{u} \mathbf{H} \mathbf{R}_{u}^{\top}, \overline{\mathbf{e}}_{p}=$ $\left(\mathbf{I}_{3}-\overline{\mathbf{H}}\right) \overline{\mathbf{m}}^{\star}$, and $\dot{\overline{\mathbf{R}}}=\overline{\mathbf{R}} \overline{\mathbf{\Omega}}_{\times}$.

Since the inner-loop controller ensures the (almost) global convergence of $\mathbf{R}^{\top} \mathbf{u}$ to $\mathbf{u}$, one deduces that $\overline{\mathbf{R}}^{\top} \mathbf{e}_{3} \rightarrow \mathbf{e}_{3}$, which in turn implies the convergence

$$
\overline{\mathbf{R}} \rightarrow\left[\begin{array}{ccc}
C(\psi) & -S(\psi) & 0 \\
S(\psi) & C(\psi) & 0 \\
0 & 0 & 1
\end{array}\right]
$$

for some scalar $\psi$. From (24), it is straightforward that $\omega_{r}$ and $\dot{\omega}_{r}$ remain bounded by $k_{\Theta 1} \Delta_{\omega} / k_{\Theta 2}$ and $2 k_{\Theta 1} \Delta_{\omega}$, respectively. The boundedness of $\omega_{r}$ and $\dot{\omega}_{r}$ is a necessary condition of Propositions 3. Then, the convergence of $\mathbf{e}_{p}$ and, thus, of $\overline{\mathbf{e}}_{p}$ to zero as a result of Proposition 1 implies that

$$
\overline{\mathbf{p}}_{C} \rightarrow \frac{1}{a^{\star}}\left(\mathbf{I}_{3}-\overline{\mathbf{R}}\right) \overline{\mathbf{m}}^{\star} \rightarrow\left[\begin{array}{c}
\frac{\bar{m}_{1}^{\star}}{a^{\star}}(1-C(\psi)) \\
-\frac{\bar{m}_{1}^{\star}}{a^{\star}} S(\psi) \\
0
\end{array}\right]
$$

with $\bar{m}_{1}^{\star}$ the first component of $\overline{\mathbf{m}}^{\star}$. Using (47) and (48), one can verify that

$\bar{h}_{1,2} \rightarrow S(\psi)+\frac{\bar{m}_{1}^{\star} \bar{n}_{2}^{\star}}{\mathbf{n}^{\star} \mathbf{m}^{\star}}(1-C(\psi)) \rightarrow \frac{1}{C\left(\psi^{\star}\right)}\left(S\left(\psi+\psi^{\star}\right)-S\left(\psi^{\star}\right)\right)$,

with $\psi^{\star} \triangleq \operatorname{asin}\left(\frac{-\bar{m}_{1}^{\star} \bar{n}_{2}^{\star}}{\sqrt{\left(\mathbf{n}^{\star \top} \mathbf{m}^{\star}\right)^{2}+\left(\bar{m}_{1}^{\star} \bar{n}_{2}^{\star}\right)^{2}}}\right) \in(-\pi / 2, \pi / 2)$ and $\bar{n}_{2}^{\star}$ the second component of $\overline{\mathbf{n}}^{\star}$.

Denoting $\omega \triangleq \Omega^{\top} \mathbf{u}$, one verifies that $\dot{\psi} \rightarrow \omega \rightarrow \omega_{r}$. Then, if $\Delta_{\omega}$ is large enough such that $\Delta_{\omega}>\bar{\Delta}_{\omega} \triangleq \frac{1}{C\left(\psi^{\star}\right)}(1-$ $\left.S\left(\psi^{\star}\right)\right)$, the zero dynamics of $(\psi, \omega)$ are given by

$$
\left\{\begin{array}{l}
\dot{\psi}=\omega \\
\dot{\omega}=-k_{\Theta 2} \omega-\frac{k_{\Theta 1}}{C\left(\psi^{\star}\right)}\left(S\left(\psi+\psi^{\star}\right)-S\left(\psi^{\star}\right)\right)
\end{array}\right.
$$

By simple changes of variables $\tau \triangleq \sqrt{k_{\Theta 1}} t, \bar{\omega} \triangleq \omega / \sqrt{k_{\Theta 1}}$, and $\kappa \triangleq k_{\Theta 2} / \sqrt{k_{\Theta 1}}$, 50, can be rewritten as

$$
\left\{\begin{array}{l}
\frac{d \psi}{d \tau}=\bar{\omega} \\
\frac{d \bar{\omega}}{d \tau}=-\kappa \bar{\omega}-\frac{1}{C\left(\psi^{\star}\right)}\left(S\left(\psi+\psi^{\star}\right)-S\left(\psi^{\star}\right)\right)
\end{array}\right.
$$

which has identical form as 52 . From here, let $\bar{\kappa}$ be specified as in the proof of the technical lemma 6 As a result of the technical lemma 5. $(\psi, \omega)$ converges to either $(2 k \pi, 0)$ or $\left(\pi-2 \psi^{\star}+2 k \pi, 0\right)$ for some integer $k$. By analyzing the linearised system of (50) about these equilibria, it is easy to prove that the "desired" equilibrium $(\psi, \omega)=(2 k \pi, 0)$ is stable and the "undesired" equilibrium $(\psi, \omega)=\left(\pi-2 \psi^{\star}+2 k \pi, 0\right)$ is unstable. The convergence of $\psi$ to $2 k \pi$ (for some integer $k$ ) almost globally, combined with (47) and (48), ensures that $\mathbf{H}$ converges to $\mathbf{I}_{3}$ for almost all initial conditions.

Lemma 5. Consider the system

$$
\left\{\begin{array}{l}
\dot{\psi}=\omega \\
\dot{\omega}=-\kappa \omega-\frac{1}{C\left(\psi^{\star}\right)}\left(S\left(\psi+\psi^{\star}\right)-S\left(\psi^{\star}\right)\right)
\end{array}\right.
$$

with $\psi, \omega \in \mathbb{R}, \kappa>0$ and $\left|\psi^{\star}\right|<\frac{\pi}{2}$. There exists $\bar{\kappa}>0$ such that $\forall \kappa>\bar{\kappa}$ one ensures that $(\psi, \omega)$ converges to either $(2 k \pi, 0)$ or $\left(\pi-2 \psi^{\star}+2 k \pi, 0\right)$ with some integer $k$.

Proof: One verifies that

$$
\left|\frac{1}{C\left(\psi^{\star}\right)}\left(S\left(\psi+\psi^{\star}\right)-S\left(\psi^{\star}\right)\right)\right| \leq \frac{2}{C\left(\psi^{\star}\right)}-\frac{1-\left|S\left(\psi^{\star}\right)\right|}{C\left(\psi^{\star}\right)}<\frac{2}{C\left(\psi^{\star}\right)} .
$$

Then, from $52 \mathrm{~b}$ one deduces the existence of a time instant $T_{1} \geq 0$ such that $\left|\omega\left(t \geq T_{1}\right)\right| \leq \frac{2}{\kappa C\left(\psi^{\star}\right)}$. Now, let us consider the two possible cases.

Case 1: Assume that there exists a time instant $T_{2} \geq T_{1}$ such that $\psi\left(T_{2}\right)=2 k \pi$ for some integer $k$. Let $\bar{\kappa}$ be specified as in the proof of Lemma 6 (i.e. (65)). Then, as a result of Lemma 6 one ensures that $\lim _{t \rightarrow \infty} \psi(t)=2 k \pi$ and $\lim _{t \rightarrow \infty} \omega(t)=0$.

Case 2: Assume that there does not exist any time instant $T_{2} \geq T_{1}$ such that $\psi\left(T_{2}\right)=2 k \pi$ for some integer $k$. This implies that for all $t \geq T_{1}$ there exists an integer $k$ such that

$$
2 k \pi<\psi\left(t \geq T_{1}\right)<2 k \pi+2 \pi \text {. }
$$

This in turn implies that the candidate Lyapunov function

$$
\mathcal{U} \triangleq \frac{1}{2} \omega^{2}+\frac{1}{C\left(\psi^{\star}\right)}\left(1-C\left(\psi+\psi^{\star}\right)\right)+T\left(\psi^{\star}\right)\left(a_{\psi^{\star}}-\psi\right),
$$

with $a_{\psi^{\star}}=2 k \pi+2 \pi$ if $\psi^{\star} \geq 0$ and $a_{\psi^{\star}}=2 k \pi$ if $\psi^{\star}<0$, is positive definite for all $t \geq T_{1}$. From (53) and (52), one verifies that

$$
\dot{\mathcal{U}}\left(t \geq T_{1}\right)=-\kappa \omega^{2} \leq 0 .
$$

By application of LaSalle's theorem, one deduces the convergence of $\dot{\mathcal{U}}$ and, thus, of $\omega$ to zero. Then, one deduces the convergence of $\dot{\omega}$ to zero, which in turn ensures the convergence of $S\left(\psi+\psi^{\star}\right)-S\left(\psi^{\star}\right)$ to zero. This means that $\psi$ converges to either $2 k \pi$ (without overshoot) or $\pi-2 \psi^{\star}+2 k \pi$ with some integer $k$.

Lemma 6. Consider system (52) with positive gain $\kappa,\left|\psi^{\star}\right|<$ $\frac{\pi}{2}$, and initial conditions $\left|\omega\left(t_{0}\right)\right| \leq \frac{2}{\kappa C\left(\psi^{\star}\right)}, \psi\left(t_{0}\right)=2 k \pi$ with some integer $k$. There exist two positive numbers $\bar{\kappa}$ and $\varepsilon$ (whose expressions are given in the proof) such that $\forall \kappa>\bar{\kappa}$ one has $\psi\left(t \geq t_{0}\right) \in \mathcal{B}_{\varepsilon} \triangleq\{\psi(t) \in \mathbb{R}$ s.t. $|\psi(t)-2 k \pi| \leq \varepsilon\}$. Moreover, $\lim _{t \rightarrow \infty} \psi(t)=2 k \pi$ and $\lim _{t \rightarrow \infty} \omega(t)=0$. 
Proof: Let us make the proof for the case where $0 \leq$ $\psi^{\star}<\pi / 2$. The proof for the case where $-\pi / 2<\psi^{\star} \leq$ 0 will proceed analogously with the changes of variables $\bar{\psi}^{\star}=-\psi^{\star}, \bar{\psi}=-\psi, \bar{\omega}=-\omega$. Now, consider the following candidate Lyapunov function

$$
\begin{aligned}
\mathcal{V} & \triangleq \frac{1}{2} \omega^{2}+\alpha(1-C(\psi))+\frac{1}{\bar{\kappa}} \omega\left(S\left(\psi+\psi^{\star}\right)-S\left(\psi^{\star}\right)\right) \\
& =\frac{1}{2} \omega^{2}+2 \alpha S^{2}(\psi / 2)+\frac{2}{\bar{\kappa}} \omega S(\psi / 2) C\left(\psi / 2+\psi^{\star}\right) \\
& \geq 2\left(\alpha-1 / \bar{\kappa}^{2}\right) S^{2}(\psi / 2) .
\end{aligned}
$$

with $\bar{\kappa} \triangleq \kappa C\left(\psi^{\star}\right)$ and a positive number $\alpha$ to be determined hereafter. The function $\mathcal{V}$ is positive definite if $\alpha>1 / \bar{\kappa}^{2}$. One verifies that

$$
\mathcal{V}\left(t_{0}\right) \leq 2 / \bar{\kappa}^{2} \text {. }
$$

Define $\Omega_{\mathcal{B}_{\varepsilon}}$ as the contour of $\mathcal{B}_{\varepsilon}$, so that $S\left(\frac{\psi}{2}\right)= \pm S\left(\frac{\varepsilon}{2}\right)$ for all $\psi \in \Omega_{\mathcal{B}_{\varepsilon}}$. We will settle sufficient conditions on $\kappa$ and $\varepsilon$ so that $\mathcal{V}\left(\psi \in \Omega_{\mathcal{B}_{\varepsilon}}\right)>\mathcal{V}\left(t_{0}\right)$ and $\dot{\mathcal{V}}\left(\psi \in \mathcal{B}_{\varepsilon}\right) \leq 0$. This ensures that $\mathcal{V}$ is non-increasing when $\psi$ remains in the domain $\mathcal{B}_{\varepsilon}$ and, subsequently, that $\psi(t) \in \mathcal{B}_{\varepsilon}$ for all $t \geq t_{0}$.

First, one verifies from (55), (56) that $\mathcal{V}\left(\psi \in \Omega_{\mathcal{B}_{\varepsilon}}\right)>\mathcal{V}\left(t_{0}\right)$ if $2\left(\alpha-1 / \bar{\kappa}^{2}\right) S^{2}(\varepsilon / 2)>2 / \bar{\kappa}^{2}$, which can be equivalently written as

$$
\alpha>\alpha_{\varepsilon} \triangleq \frac{1}{\bar{\kappa}^{2}}\left(1+\frac{1}{S^{2}(\varepsilon / 2)}\right) .
$$

We now find the condition for which $\dot{\mathcal{V}}\left(\psi \in \mathcal{B}_{\varepsilon}\right) \leq 0$. It can be verified that

$$
\begin{aligned}
\dot{\mathcal{V}}= & -\left(\frac{\bar{\kappa}}{C\left(\psi^{\star}\right)}-\frac{C\left(\psi+\psi^{\star}\right)}{\bar{\kappa}}\right) \omega^{2} \\
& -\frac{1}{\bar{\kappa} C\left(\psi^{\star}\right)}\left(S\left(\psi+\psi^{\star}\right)-S\left(\psi^{\star}\right)\right)^{2} \\
& +\left(\frac{\alpha C(\psi / 2)}{C\left(\psi / 2+\psi^{\star}\right)}-\frac{2}{C \psi^{\star}}\right)\left(S\left(\psi+\psi^{\star}\right)-S\left(\psi^{\star}\right)\right) \omega
\end{aligned}
$$

From here, one ensures that $\dot{\mathcal{V}}\left(\psi \in \mathcal{B}_{\varepsilon}\right) \leq 0$ if

$$
\begin{aligned}
& \frac{4}{\bar{\kappa} C\left(\psi^{\star}\right)}\left(\frac{\bar{\kappa}}{C\left(\psi^{\star}\right)}-\frac{C\left(\psi+\psi^{\star}\right)}{\bar{\kappa}}\right)>\left(\frac{\alpha C\left(\frac{\psi}{2}\right)}{C\left(\frac{\psi}{2}+\psi^{\star}\right)}-\frac{2}{C\left(\psi^{\star}\right)}\right)^{2} \\
\Leftrightarrow & \alpha^{2}-\frac{4 C\left(\frac{\psi}{2}+\psi^{\star}\right)}{C\left(\psi^{\star}\right) C\left(\frac{\psi}{2}\right)} \alpha+\frac{4 C\left(\psi+\psi^{\star}\right) C^{2}\left(\frac{\psi}{2}+\psi^{\star}\right)}{\bar{\kappa}^{2} C\left(\psi^{\star}\right) C^{2}\left(\frac{\psi}{2}\right)}<0 .
\end{aligned}
$$

If $\varepsilon$ is chosen such that $\varepsilon \leq \min \left(\frac{\pi}{2}, \pi-2 \psi^{\star}\right)$, one ensures

$$
C\left(\psi / 2+\psi^{\star}\right)>0, \quad C(\psi / 2)>0 .
$$

Thus, one deduces from (59) that $\alpha_{1}(\psi)<\alpha<\alpha_{2}(\psi)$, with

$$
\begin{aligned}
& \alpha_{1}(\psi) \triangleq \frac{2 C\left(\psi / 2+\psi^{\star}\right)}{C\left(\psi^{\star}\right) C(\psi / 2)}\left(1-\sqrt{1-\frac{C\left(\psi^{\star}\right) C\left(\psi+\psi^{\star}\right)}{\bar{\kappa}^{2}}}\right), \\
& \alpha_{2}(\psi) \triangleq \frac{2 C\left(\psi / 2+\psi^{\star}\right)}{C\left(\psi^{\star}\right) C(\psi / 2)}\left(1+\sqrt{1-\frac{C\left(\psi^{\star}\right) C\left(\psi+\psi^{\star}\right)}{\bar{\kappa}^{2}}}\right) .
\end{aligned}
$$

A constant solution of $\alpha$ for inequality (59) and satisfying (57) exists if

$$
\left\{\begin{aligned}
\sup _{\psi \in \mathcal{B}_{\varepsilon}} \alpha_{1}(\psi) & <\inf _{\psi \in \mathcal{B}_{\varepsilon}} \alpha_{2}(\psi) \\
\alpha_{\varepsilon} & <\inf _{\psi \in \mathcal{B}_{\varepsilon}} \alpha_{2}(\psi)
\end{aligned}\right.
$$

We now find sufficient conditions so that 60 is satisfied. One verifies that $\frac{C\left(\psi / 2+\psi^{\star}\right)}{C(\psi / 2)}=C\left(\psi^{\star}\right)-S\left(\psi^{\star}\right) T(\psi / 2)$ and, thus,

$$
\left\{\begin{array}{l}
\sup _{\psi \in \mathcal{B}_{\varepsilon}} \alpha_{1}(\psi) \leq 2\left(1-\sqrt{1-\frac{C\left(\psi^{\star}\right)}{\bar{\kappa}^{2}}}\right) \\
\inf _{\psi \in \mathcal{B}_{\varepsilon}} \alpha_{2}(\psi) \geq 2\left(1-T\left(\psi^{\star}\right) T(\varepsilon / 2)\right)\left(1+\sqrt{1-\frac{C\left(\psi^{\star}\right)}{\bar{\kappa}^{2}}}\right)
\end{array}\right.
$$

From here, one deduces that 60a is satisfied if

$$
1-\sqrt{1-\frac{C\left(\psi^{\star}\right)}{\bar{\kappa}^{2}}}<\left(1-T\left(\psi^{\star}\right) T(\varepsilon / 2)\right)\left(1+\sqrt{1-\frac{C\left(\psi^{\star}\right)}{\bar{\kappa}^{2}}}\right),
$$

which can be equivalently written as

$$
y<\frac{2\left(\cot \left(\psi^{\star}\right)-x\right)}{2 \cot \left(\psi^{\star}\right)-x}
$$

with $x \triangleq T(\varepsilon / 2)$ and $y \triangleq 1-\sqrt{1-\frac{C\left(\psi^{\star}\right)}{\bar{\kappa}^{2}}}$.
Since $\varepsilon \leq \min \left(\frac{\pi}{2}, \pi-2 \psi^{\star}\right)$, one deduces

$$
0<x \leq \min \left(1, \cot \left(\psi^{\star}\right)\right) .
$$

On the other hand, inequality $60 \mathrm{~b}$ is satisfied if

$$
\begin{aligned}
& \frac{1}{\bar{\kappa}^{2}}\left(1+\frac{1}{S^{2}\left(\frac{\varepsilon}{2}\right)}\right)<2\left(1-T\left(\psi^{\star}\right) T\left(\frac{\varepsilon}{2}\right)\right)\left(1+\sqrt{1-\frac{C\left(\psi^{\star}\right)}{\bar{\kappa}^{2}}}\right) \\
& \Leftrightarrow \quad \frac{(2-y) y}{C\left(\psi^{\star}\right)}\left(2+\frac{1}{x^{2}}\right)<2\left[1-T\left(\psi^{\star}\right) x\right](2-y) \\
& \Leftrightarrow \quad y<\frac{2 S\left(\psi^{\star}\right) x^{2}\left(\cot \left(\psi^{\star}\right)-x\right)}{2 x^{2}+1},
\end{aligned}
$$

where we have used $\frac{1}{S^{2}(\varepsilon / 2)}=1+\frac{1}{T^{2}(\varepsilon / 2)}$ and $\bar{\kappa}^{2}=\frac{C\left(\psi^{\star}\right)}{(2-y) y}$ (deduced from the definition of $y$ ).

One can easily verify that

$$
0<\frac{2 S\left(\psi^{\star}\right) x^{2}\left(\cot \left(\psi^{\star}\right)-x\right)}{2 x^{2}+1} \leq \frac{2\left(\cot \left(\psi^{\star}\right)-x\right)}{2 \cot \left(\psi^{\star}\right)-x}<1,
$$

under condition 62, which means that if inequality 63 is satisfied, then inequality (61) is also satisfied. Therefore, in summary we only need to find $x$ and $y$ satisfying

$$
\left\{\begin{array}{l}
x \in \mathcal{D}_{\psi^{\star}} \triangleq\left(0, \min \left(1, \cot \left(\psi^{\star}\right)\right)\right] \\
y<f(x)
\end{array}\right.
$$

with the function $f(x) \triangleq \frac{2 S\left(\psi^{\star}\right) x^{2}\left(\cot \left(\psi^{\star}\right)-x\right)}{2 x^{2}+1}$. The gradient of $f(x)$ is given by $\frac{\partial f(x)}{\partial x}=\frac{-4 S\left(\psi^{*}\right) x\left(x^{3}+1.5 x-\cot \left(\psi^{\star}\right)\right)}{\left(2 x^{2}+1\right)^{2}}$, which is null only at 0 and at $x_{\psi^{\star}}$ defined by

$$
x_{\psi^{\star}} \triangleq\left(\frac{\sqrt{\cot ^{2}\left(\psi^{\star}\right)+0.5}+\cot \left(\psi^{\star}\right)}{2}\right)^{1 / 3}-\left(\frac{\sqrt{\cot ^{2}\left(\psi^{\star}\right)+0.5}-\cot \left(\psi^{\star}\right)}{2}\right)^{1 / 3}
$$

One can verify that $0<x_{\psi^{\star}}<\cot \left(\psi^{\star}\right)$. From here, since $f(x)$ is an increasing function in $\left[0, x_{\psi^{\star}}\right]$, one deduces that $\sup _{x \in \mathcal{D}_{\psi^{\star}}} f(x)=f\left(x^{\star}\right)$, with $x^{\star} \triangleq \min \left(1, x_{\psi^{\star}}\right)$. Therefore, by choosing $x=x^{\star}$ or equivalently $\varepsilon=2 \operatorname{atan}\left(x^{\star}\right)$, one deduces from (64) that $y<f\left(x^{\star}\right)$, yielding

$$
\kappa>\bar{\kappa} \triangleq \frac{1}{\sqrt{C\left(\psi^{\star}\right) f\left(x^{\star}\right)\left(2-f\left(x^{\star}\right)\right)}} .
$$

We have established sufficient conditions on $\kappa$ (i.e. (65) ) and on $\varepsilon$ (i.e. $\varepsilon=2 \operatorname{atan}\left(x^{\star}\right)$ ) so that $\mathcal{V}\left(\psi \in \Omega_{\mathcal{B}_{\varepsilon}}\right)>\mathcal{V}\left(t_{0}\right)$ and $\dot{\mathcal{V}}\left(\psi \in \mathcal{B}_{\varepsilon}\right) \leq 0$, which in turn ensure that $\psi(t) \in \mathcal{B}_{\varepsilon}$ and $\dot{\mathcal{V}}(t) \leq 0$ for all $t \geq t_{0}$. By application of LaSalle's theorem, one ensures that $\dot{\mathcal{V}}$ converges to zero, which in view of (58) and 59) ensures the convergence of $\omega$ and $S\left(\psi+\psi^{\star}\right)-S\left(\psi^{\star}\right)$ to zero. Since the equation $S\left(\psi+\psi^{\star}\right)-S\left(\psi^{\star}\right)=0$ has a unique solution of $\psi$ in $\mathcal{B}_{\varepsilon}$ that is $\psi=2 k \pi$, one deduces that $\lim _{t \rightarrow \infty} \psi(t)=2 k \pi$.

\section{APPENDIX G}

\section{PROOF OF PROPOSITION 3}

Consider the following candidate Lyapunov function

$$
\begin{aligned}
\mathcal{L} \triangleq \frac{1}{2} \widetilde{\mathbf{W}^{\top}} \mathbf{M}_{T} \widetilde{\mathbf{W}} & +\frac{1}{2}\left(\mathbf{z}_{V}-\mathbf{K}_{i V}^{-1} \boldsymbol{\Delta}_{F}\right)^{\top} \mathbf{K}_{i V}\left(\mathbf{z}_{V}-\mathbf{K}_{i V}^{-1} \boldsymbol{\Delta}_{F}\right) \\
& +\frac{1}{2}\left(\mathbf{z}_{\Omega}-\mathbf{K}_{i \Omega}^{-1} \boldsymbol{\Delta}_{\Gamma}\right)^{\top} \mathbf{K}_{i \Omega}\left(\mathbf{z}_{\Omega}-\mathbf{K}_{i \Omega}^{-1} \boldsymbol{\Delta}_{\Gamma}\right)
\end{aligned}
$$

with $\mathbf{M}_{T}>0$ given by (1) and $\widetilde{\mathbf{W}} \triangleq\left[\widetilde{\mathbf{V}}^{\top}, \widetilde{\boldsymbol{\Omega}}^{\top}\right]^{\top}$. Using

$$
\begin{aligned}
& \widetilde{\boldsymbol{\Omega}}^{\top}(\mathbf{J} \boldsymbol{\Omega}+\mathbf{D V})_{\times} \widetilde{\boldsymbol{\Omega}}=0, \\
& \tilde{\mathbf{V}}^{\top}\left(\mathbf{M V}+\mathbf{D}^{\top} \boldsymbol{\Omega}\right)_{\times} \widetilde{\boldsymbol{\Omega}}+\widetilde{\boldsymbol{\Omega}}^{\top}\left(\mathbf{M} \mathbf{V}+\mathbf{D}^{\top} \boldsymbol{\Omega}\right)_{\times} \widetilde{\mathbf{V}}=0, \\
& \widetilde{\boldsymbol{\Omega}}^{\top}(\mathbf{M} \widetilde{\mathbf{V}})_{\times} \mathbf{V}_{r}=-\widetilde{\mathbf{V}}^{\top} \mathbf{M}\left(\widetilde{\boldsymbol{\Omega}} \times \mathbf{V}_{r}\right), \\
& \widetilde{\boldsymbol{\Omega}}^{\top}(\mathbf{D} \widetilde{\mathbf{V}})_{\times} \boldsymbol{\Omega}_{r}=-\widetilde{\mathbf{V}}^{\top} \mathbf{D}^{\top}\left(\widetilde{\boldsymbol{\Omega}} \times \boldsymbol{\Omega}_{r}\right),
\end{aligned}
$$

and (27) and (28), one verifies that 


$$
\begin{aligned}
& \dot{\mathcal{L}}=\widetilde{\mathbf{V}}^{\top}\left(\mathbf{M} \dot{\tilde{\mathbf{V}}}+\mathbf{D}^{\top} \dot{\widetilde{\mathbf{\Omega}}}+\mathbf{K}_{i V} \mathbf{z}_{V}-\boldsymbol{\Delta}_{F}\right) \\
& +\widetilde{\boldsymbol{\Omega}}^{\top}\left(\mathbf{J} \dot{\widetilde{\mathbf{\Omega}}}+\mathbf{D} \dot{\tilde{\mathbf{V}}}+\mathbf{K}_{i \Omega} \mathbf{z}_{\Omega}-\boldsymbol{\Delta}_{\Gamma}\right) \\
& =\widetilde{\mathbf{V}}^{\top}\left[\left(\mathbf{M} \tilde{\mathbf{V}}+\mathbf{D}^{\top} \widetilde{\boldsymbol{\Omega}}\right)_{\times} \boldsymbol{\Omega}_{r}-\mathbf{M}\left(\widetilde{\boldsymbol{\Omega}} \times \mathbf{V}_{r}\right)-\mathbf{D}^{\top}\left(\widetilde{\boldsymbol{\Omega}} \times \boldsymbol{\Omega}_{r}\right)\right. \\
& \left.+\mathbf{F}_{G B}+\overline{\mathbf{F}}_{d}+\mathbf{F}_{r}+\mathbf{F}_{c}+\mathbf{K}_{i V} \mathbf{z}_{V}\right] \\
& +\widetilde{\boldsymbol{\Omega}}^{\top}\left[(\mathbf{J} \widetilde{\boldsymbol{\Omega}})_{\times} \boldsymbol{\Omega}_{r}+\left(\mathbf{D}^{\top} \widetilde{\boldsymbol{\Omega}}\right)_{\times} \mathbf{V}_{r}\right. \\
& \left.+m g \mathbf{r}_{G} \times \mathbf{R}^{\top} \mathbf{e}_{g}+\boldsymbol{\Gamma}_{d}+\boldsymbol{\Gamma}_{r}+\boldsymbol{\Gamma}_{c}+\mathbf{K}_{i \Omega} \mathbf{z}_{\Omega}\right] \\
& =-\widetilde{\mathbf{V}}^{\top}\left(\mathbf{K}_{V}+\mathbf{D}_{V l}+|\mathbf{V}| \mathbf{D}_{V q}\right) \widetilde{\mathbf{V}} \\
& -\widetilde{\boldsymbol{\Omega}}^{\top}\left(\mathbf{K}_{\Omega}+\mathbf{D}_{\Omega l}+|\boldsymbol{\Omega}| \mathbf{D}_{\Omega q}\right) \widetilde{\boldsymbol{\Omega}} \leq 0
\end{aligned}
$$

Since $\dot{\mathcal{L}}$ is negative semi-definite, $\widetilde{\mathbf{V}}, \widetilde{\Omega}, \mathbf{z}_{V}$, and $\mathbf{z}_{\Omega}$ are bounded. Since $\mathbf{V}_{r}$ and $\boldsymbol{\Omega}_{r}$ and their derivative are bounded (consequences of Proposition 1 and Proposition 2 or Theorem 1), one easily deduces from 27 that $\dot{\tilde{\mathbf{V}}}$ and $\dot{\widetilde{\Omega}}$ are also bounded. Then, one deduces that $\ddot{\mathcal{L}}$ is also bounded, which implies the uniform continuity of $\dot{\mathcal{L}}$. From here, by application of Barbalat's lemma one deduces the convergence of $\dot{\mathcal{L}}$ and, thus, $\widetilde{\mathbf{V}}$ and $\widetilde{\Omega}$ to zero. Then, using Barbalat-like arguments one ensures that $\dot{\overrightarrow{\mathbf{V}}}$ and $\dot{\widetilde{\Omega}}$ also converge to zero, implying that $\mathbf{z}_{V}$ and $\mathbf{z}_{\Omega}$ converge, respectively, to $\mathbf{z}_{V}^{\star} \triangleq \mathbf{K}_{i V}^{-1} \boldsymbol{\Delta}_{F}$ and $\mathbf{z}_{\Omega}^{\star} \triangleq \mathbf{K}_{i \Omega}^{-1} \boldsymbol{\Delta}_{\Gamma}$. We have proved that the equilibrium $\left(\mathbf{V}, \boldsymbol{\Omega}, \mathbf{z}_{V}, \mathbf{z}_{\Omega}\right)=\left(\mathbf{V}_{r}, \boldsymbol{\Omega}_{r}, \mathbf{z}_{V}^{\star}, \mathbf{z}_{\Omega}^{\star}\right)$ is GAS. It remains to show that this equilibrium is also LES. To this purpose, it suffices to study the linearised closed-loop system of 27):

$$
\left\{\begin{aligned}
\mathbf{M} \dot{\tilde{\mathbf{V}}}+\mathbf{D}^{\top} \dot{\tilde{\mathbf{\Omega}}} & =-\left(\mathbf{K}_{V}+\mathbf{D}_{V l}\right) \widetilde{\mathbf{V}}-\mathbf{K}_{i V} \mathbf{z}_{V}+\boldsymbol{\Delta}_{F} \\
\mathbf{J} \dot{\widetilde{\mathbf{\Omega}}}+\mathbf{D} \dot{\tilde{\mathbf{V}}} & =-\left(\mathbf{K}_{\Omega}+\mathbf{D}_{\Omega}\right) \widetilde{\mathbf{\Omega}}-\mathbf{K}_{i \Omega} \mathbf{z}_{\Omega}+\boldsymbol{\Delta}_{\Gamma} \\
\dot{\mathbf{z}}_{V} & =\widetilde{\mathbf{V}} \\
\dot{\mathbf{z}}_{\Omega} & =\widetilde{\boldsymbol{\Omega}}
\end{aligned}\right.
$$

Using the same Lyapunov candidate function $\mathcal{L}$ defined by (66) and the linearized error dynamics (68) one verifies that

$$
\dot{\mathcal{L}}=-\widetilde{\mathbf{V}}^{\top}\left(\mathbf{K}_{V}+\mathbf{D}_{V l}\right) \widetilde{\mathbf{V}}-\widetilde{\boldsymbol{\Omega}}^{\top}\left(\mathbf{K}_{\Omega}+\mathbf{D}_{\Omega l}\right) \widetilde{\boldsymbol{\Omega}} \leq 0 .
$$

From here, direct application of LaSalle's theorem ensures the convergence of $\widetilde{\mathbf{V}}, \widetilde{\Omega}$, and of $\dot{\widetilde{\mathbf{V}}}$ and $\dot{\widetilde{\Omega}}$ to zero, which in turn implies the convergence of $\mathbf{z}_{V}$ and $\mathbf{z}_{\Omega}$ to $\mathbf{z}_{V}^{\star}$ and $\mathbf{z}_{\Omega}^{\star}$, respectively. Since the equilibrium $\left(\mathbf{V}, \boldsymbol{\Omega}, \mathbf{z}_{V}, \mathbf{z}_{\Omega}\right)=$ $\left(\mathbf{V}_{r}, \boldsymbol{\Omega}_{r}, \mathbf{z}_{V}^{\star}, \mathbf{z}_{\Omega}^{\star}\right)$ of the linear system $(68)$ is stable, it is LES.

Now, for the case where $\boldsymbol{\Omega}_{r}$ is defined by $250+24$ (Theorem 1), we now prove the convergence of $\mathbf{R}^{\top} \mathbf{e}_{g}$ to either $\mathbf{e}_{g}$ or $-\mathbf{e}_{g}$. Since $\widetilde{\boldsymbol{\Omega}}$ converges exponentially to zero, at zero dynamics (i.e. $\boldsymbol{\Omega} \equiv \boldsymbol{\Omega}_{r}$ ) the derivative of the following positive function $\mathcal{S}_{0} \triangleq 1-\mathbf{e}_{g}^{\top} \mathbf{R}^{\top} \mathbf{e}_{g}$ satisfies

$$
\dot{\mathcal{S}}_{0}=-\boldsymbol{\Omega}_{r}^{\top}\left(\mathbf{e}_{g} \times \mathbf{R}^{\top} \mathbf{e}_{g}\right)=-k_{u}\left|\mathbf{e}_{g} \times \mathbf{R} \mathbf{e}_{g}\right|^{2} \leq 0,
$$

which allows one to deduce the convergence of $\mathbf{e}_{g} \times \mathbf{R}^{\top} \mathbf{e}_{g}$ to zero, or equivalently the convergence of $\mathbf{R}^{\top} \mathbf{e}_{g}$ to $\pm \mathbf{e}_{g}$.

By denoting $\Theta$ the angle between $\mathbf{R}^{\top} \mathbf{e}_{g}$ and $\mathbf{e}_{g}$, i.e., $C(\Theta)=\mathbf{e}_{g}^{\top} \mathbf{R e}_{g}$, around a small neighborhood of the equilibrium $\left(\mathbf{V}, \boldsymbol{\Omega}, \mathbf{z}_{V}, \mathbf{z}_{\Omega}, \mathbf{R}^{\top} \mathbf{e}_{g}\right)=\left(\mathbf{V}_{r}, \boldsymbol{\Omega}_{r}, \mathbf{z}_{V}^{\star}, \mathbf{z}_{\Omega}^{\star}, \mathbf{e}_{g}\right)$, one has $\mathcal{S}_{0} \approx 0.5 \Theta^{2}$ and $\dot{\mathcal{S}}_{0} \leq-2 k_{u} \mathcal{S}_{0}+|\widetilde{\boldsymbol{\Omega}}|$. From here, one deduces that locally $\mathcal{S}_{0}$ and $\Theta$ converge exponentially to zero, using the exponential convergence of $\widetilde{\boldsymbol{\Omega}}$ to zero. Thus, the equilibrium $\left(\mathbf{V}, \boldsymbol{\Omega}, \mathbf{z}_{V}, \mathbf{z}_{\Omega}, \mathbf{R}^{\top} \mathbf{e}_{g}\right)=\left(\mathbf{V}_{r}, \boldsymbol{\Omega}_{r}, \mathbf{z}_{V}^{\star}, \mathbf{z}_{\Omega}^{\star}, \mathbf{e}_{g}\right)$ is LES.

The proof of instability of the equilibrium $\left(\mathbf{V}, \boldsymbol{\Omega}, \mathbf{z}_{V}, \mathbf{z}_{\Omega}, \mathbf{R}^{\top} \mathbf{e}_{g}\right)=\left(\mathbf{V}_{r}, \boldsymbol{\Omega}_{r}, \mathbf{z}_{V}^{\star}, \mathbf{z}_{\Omega}^{\star},-\mathbf{e}_{g}\right)$ is based on the Chetaev's theorem. Define $\mathbf{y} \triangleq \mathbf{e}_{g}+\mathbf{R}^{\top} \mathbf{e}_{g}$ and consider the function $\mathcal{S}_{1}(\mathbf{y}) \triangleq \mathbf{y}^{\top} \mathbf{e}_{g}=1+\mathbf{e}_{g}^{\top} \mathbf{R} \mathbf{e}_{g} \geq 0$, which is null at the origin, i.e., $\mathcal{S}_{1}(\mathbf{0})=0$. For some positive number $0<r<1$, define a set $U_{r} \triangleq\left\{\mathbf{y}\left|\mathcal{S}_{1}(\mathbf{y})>0,\right| \mathbf{y} \mid<r\right\}$, and note that $U_{r}$ is non-empty. By neglecting all high-order terms, the derivative of $\mathcal{S}_{1}$ can be approximately given by

$$
\dot{\mathcal{S}}_{1} \approx \mathbf{e}_{g}^{\top} \mathbf{R} \boldsymbol{\Omega}_{r \times} \mathbf{e}_{g}=k_{u}\left|\mathbf{e}_{g} \times \mathbf{R}^{\top} \mathbf{e}_{g}\right|^{2}=k_{u}\left|\mathbf{e}_{g} \times \mathbf{y}\right|^{2} .
$$

For all $\mathbf{y} \in U_{r}$, the positivity of $\mathbf{y}^{\top} \mathbf{e}_{g}$ is equivalent to the positivity of $\left|\mathbf{e}_{g} \times \mathbf{y}\right|^{2}$, which in turn ensures that $\dot{\mathcal{S}}_{1}>0$. Since all the conditions of Chetaev's theorem are satisfied [19], the origin $\mathbf{y}=\mathbf{0}$ of the linearised system is unstable.

\section{APPENDIX H}

\section{PROOF OF COROLlary 1}

Using the assumption that $\tilde{\omega}_{1}=\omega_{1}=\omega_{1 r}=0$ and following the same lines of computations as in 677 one verifies that the time-derivative of the following Lyapunov candidate function

$$
\begin{aligned}
\mathcal{L} \triangleq \frac{1}{2} \widetilde{\mathbf{W}} \mathbf{M}_{T} \widetilde{\mathbf{W}} & +\frac{1}{2}\left(\mathbf{z}_{V}-\mathbf{K}_{i V}^{-1} \boldsymbol{\Delta}_{F}\right)^{\top} \mathbf{K}_{i V}\left(\mathbf{z}_{V}-\mathbf{K}_{i V}^{-1} \boldsymbol{\Delta}_{F}\right) \\
& +\frac{1}{2}\left(\mathbf{z}_{\Omega}-\mathbf{K}_{i \Omega}^{-1} \boldsymbol{\Delta}_{\Gamma, 2,3}\right)^{\top} \mathbf{K}_{i \Omega}\left(\mathbf{z}_{\Omega}-\mathbf{K}_{i \Omega}^{-1} \boldsymbol{\Delta}_{\Gamma, 2,3}\right)
\end{aligned}
$$

is given by

$$
\begin{aligned}
\dot{\mathcal{L}}= & -\widetilde{\mathbf{V}}^{\top}\left(\mathbf{K}_{V}+\mathbf{D}_{V l}+|\mathbf{V}| \mathbf{D}_{V q}\right) \widetilde{\mathbf{V}} \\
& -\widetilde{\boldsymbol{\Omega}}_{2,3}^{\top} \mathbf{K}_{\Omega} \widetilde{\boldsymbol{\Omega}}_{2,3}-\widetilde{\boldsymbol{\Omega}}^{\top}\left(\mathbf{D}_{\Omega l}+|\boldsymbol{\Omega}| \mathbf{D}_{\Omega q}\right) \widetilde{\boldsymbol{\Omega}} \leq 0
\end{aligned}
$$

From here, the remainder of proof proceeds analogously to the proof of Proposition 3

\section{REFERENCES}

[1] A. P. Aguiar and A. M. Pascoal. Dynamic positioning and way-point tracking of underactuated AUVs in the presence of ocean currents. Int. J. of Control, 80(7):1092-1108, 2007.

[2] B. Allen, R. Stokey, T. Austin, N. Forrester, R. Goldsborough, M. Purcell, and C. von Alt. REMUS: a small, low cost AUV; system description, field trials and performance results. In MTS/IEEE OCEANS'97, pages 994-1000, 1997.

[3] J. Antich and A. Ortiz. Development of the control architecture of an underwater cable tracker. Int. J. of Intelligent Systems, 20(5):477-498, 2005.

[4] G. Antonelli. On the use of adaptive/integral actions for six-degrees-offreedom control of autonomous underwater vehicles. IEEE J. of Oceanic Engineering, 32(2):300-312, 2007.

[5] S. Benhimane and E. Malis. Homography-based 2d visual tracking and servoing. Int. J. of Robotics Research, 26(7):661-676, 2007.

[6] S. P. Bhat and D. S. Bernstein. A topological obstruction to continuous global stabilization of rotational motion and the unwinding phenomenon. Systems \& Control Letters, 39(1):63-70, 2000.

[7] L. Brignone, M. Perrier, and C. Viala. A fully autonomous docking strategy for intervention AUVs. In MTS/IEEE OCEANS'07, pages 1-6, 2007.

[8] F. Chaumette and S. Hutchinson. Visual servo control, Part I: Basic approaches. IEEE Robotics and Automation Mag., 13(4):82-90, 2006.

[9] F. Chaumette and S. Hutchinson. Visual servo control, Part II: Advanced approaches. IEEE Robotics and Automation Mag., 14(1):109-118, 2007.

[10] T. I. Fossen. Marine Control Systems. Marine Cybernetix AS, 2002.

[11] D. Fryxell, P. Oliveira, A. Pascoal, C. Silvestre, and I. Kaminer. Navigation, guidance and control of AUVs: An application to the MARIUS vehicle. Control Engineering Practice, 4:401-409, 1996.

[12] R. Garcia, J. Batlle, X. Cufi, and J. Amat. Positioning an underwater vehicle through image mosaicking. In IEEE Int. Conf. on Robotics \& Automation, 2001.

[13] T. F. Gonçalves, J. R. Azinheira, and P. Rives. Vision-based autonomous approach and landing for an aircraft using a direct visual tracking method. In Int. Conf. on Informatics in Control, Automation and Robotics, pages 94-101, 2009.

[14] T. Hamel and R. Mahony. Visual servoing of an under-actuated dynamic rigid-body system: an image-based approach. IEEE Trans. on Robotics and Automation, 18(2):187-198, 2002.

[15] M.-D. Hua, G. Allibert, S. Krupínski, and T. Hamel. Homographybased visual servoing for autonomous underwater vehicles. In IFAC World Congress, pages 5729-5733, 2014.

[16] M.-D. Hua, T. Hamel, P. Morin, and C. Samson. A control approach for thrust-propelled underactuated vehicles and its application to VTOL drones. IEEE Trans. on Automatic Control, 54(8):1837-1853, 2009. 
[17] M.-D. Hua, P. Martin, and T. Hamel. Stability analysis of velocity-aided attitude observers for accelerated vehicles. Automatica, 63:11-15, 2016.

[18] T. M. Josserand. Optimally-robust nonlinear control of a class of robotic underwater vehicles. Ph.d. thesis, University of Texas at Austin, 2006.

[19] H. K. Khalil. Nonlinear systems (3rd Edition). Prentice Hall, 2002.

[20] S. Krupínski, G. Allibert, M.-D. Hua, and T. Hamel. Pipeline tracking for fully-actuated autonomous underwater vehicle using visual servo control. In American Control Conference (ACC), pages 6196-6202, 2012.

[21] S. Krupínski, R. Desouche, N. Palomeras, G. Allibert, and M.-D. Hua Pool testing of auv visual servoing for autonomous inspection. In 4 th IFAC Workshop on Navigation, Guidance and Control of Underwater Vehicles, IFAC-PapersOnLine, volume 48, pages 274-280, 2015.

[22] H. Lamb. Hydrodynamics. Cambridge Univ. Press, 1932.

[23] L. Lapierre, D. Soetanto, and A. Pascoal. Robust nonlinear pathfollowing control of an AUV. IEEE J. of Oceanic Engineering, 33:89$102,2008$.

[24] N. E. Leonard. Stability of a bottom-heavy underwater vehicle. Automatica, 33(3):331-246, 1997.

[25] J.-E Lots, D. M. Lane, E. Trucco, and F. Chaumette. A 2-D visual servoing for underwater vehicle station keeping. In IEEE Int. Conf. on Robotics and Automation (ICRA), pages 2767-2772, 2001.

[26] E. Malis, F. Chaumette, and S. Boudet. $21 / 2 \mathrm{D}$ visual servoing. IEEE Trans. on Robotics and Automation, 15(2):238-250, 1999.

[27] G. Marani, S. K. Choi, and J. Yuh. Underwater autonomous manipulation for intervention missions AUVs. IEEE J. of Ocean Engineering, 36(1):15-23, 2009.

[28] N. Metni, T. Hamel, and F. Derkx. Visual tracking control of aerial robotic systems with adaptive depth estimation. In IEEE Conf. on Decision and Control (CDC), pages 6078-6084, 2005.

[29] A. Micaelli and C. Samson. Trajectory tracking for unicycle-type and two-steering-wheels mobile robots. Technical report, INRIA, 1993.

[30] W. Naeem, R. Sutton, S. M. Ahmad, and R. S. Burns. A review of guidance laws applicable to unmanned underwater vehicles. The $J$. of Navigation, 56(1):15-29, 2003.

[31] N. Palomeras, S. Nagappa, D. Ribas, N. Gracias, and M. Carreras. Vision-based localization and mapping system for AUV intervention. In MTS/IEEE OCEANS'13, pages 1-7, 2013.

[32] H. Plinval, P. Morin, P. Mouyon, and T. Hamel. Visual servoing for underactuated VTOL UAVs: a linear, homography-based framework. Int. J. of Robust and Nonlinear Control, 24(16):2285-2308, 2014.

[33] S. Reed, Y. Petillot, and J. Bell. An automatic approach to the detection and extraction of mine features in sidescan sonar. IEEE J. of Oceanic Engineering, 28(1):90-105, 2003.

[34] J. E. Refsnes, A. J. Sorensen, and K. Y. Pettersen. Model-based output feedback control of slender-body underactuated AUVs: theory and experiments. IEEE Trans. on Cont. Syst. Technology, 16(5):930946, 2008.

[35] F. Repoulias and E. Papadopoulos. Planar trajectory planning and tracking control design for underactuated AUVs. Ocean Engineering, 34(11):1650-1667, 2007.

[36] D. Ribas, N. Palomeras, P. Ridao, M. Carreras, and A. Mallios. Girona 500 AUV: From survey to intervention. IEEE/ASME Trans. on Mechatronics, 1(17):46-53, 2012.

[37] P. Ridao, M. Carreras, D. Ribas, P. J. Sanz, and G. Oliver. Intervention AUVs: The next challenge. In IFAC World Congress, pages 1214612159, 2014.

[38] P. Rives and J.-J. Borrelly. Underwater pipe inspection task using visual servoing techniques. In IEEE Int. Conf. on Intelligent Robots and Systems (IROS), pages 63-68, 1997.

[39] D. Smallwood and L. L. Whitcomb. Model-based dynamic positioning of underwater robotic vehicles: theory and experiment. IEEE J. of Oceanic Engineering, 29(1):169-186, 2004.

[40] I. Spangelo and O. Egeland. Trajectory planning and collision avoidance for underwater vehicles using optimal control. IEEE J. of Oceanic Engineering, 19:502-511, 1994.

[41] S. van der Zwaan, A. Bernardino, and J. Santos-Victor. Visual station keeping for floating robots in unstructured environments. Robotics and Autonomous Systems, 39(3):145-155, 2002.

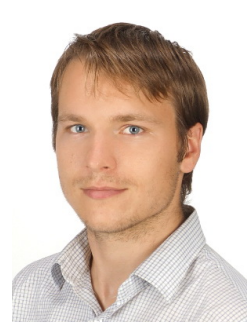

Szymon Krupínski obtained his BSc degree in Computer Science and Electrical Engineering from Jacobs University in Bremen, Germany, followed by an MSc in Naval Architecture and Marine Engineering from the University of Southampton, UK. $\mathrm{He}$ graduated with a $\mathrm{PhD}$ in Automation, Signal and Image Processing from Université Nice Sophia Antipolis in France after a stay at the I3S Laboratory of Sophia Antipolis. He currently holds a position of research engineer at Cybernetix with focus on autonomous and remotely operated robots and sensors. His core interests include navigation and positioning as well as visual and sonar-based servoing.

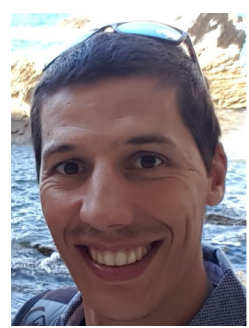

Guillaume Allibert received the M.Sc. degree from the University of Picardie Jules Verne, France, in 2005, and the Ph.D. degree in Automatic Control from the University of Orléans, France, in 2008. He is currently an Associate Professor at the I3S UCACNRS laboratory with University of Nice Sophia Antipolis, Nice, France, since 2009. His research interests include nonlinear control and nonlinear observer with applications to Unmanned Aerial Vehicles and Autonomous Underwater Vehicles.

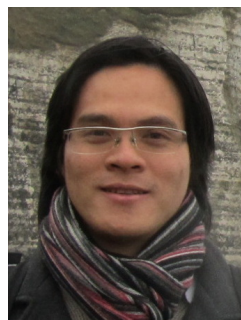

Minh-Duc Hua graduated from Ecole Polytechnique, France in 2006 and received his $\mathrm{PhD}$ degree from the University of Nice Sophia-Antipolis, France in 2009. After two postdoctoral years at the I3S laboratory in France and a short research stay at the Australian National University, he joined the French National Center for Scientific Research (CNRS) in 2011, where he presently holds a tenured research position (CR1). He was assigned to the Institute for Intelligent Systems and Robotics (ISIR UPMC-CNRS) in Paris from 2011 to 2016. Now he works at the I3S UCA-CNRS laboratory. His research interests include nonlinear control, sensor-based control, nonlinear observer, and computer vision, with applications to Unmanned Aerial Vehicles and Autonomous Underwater Vehicles.

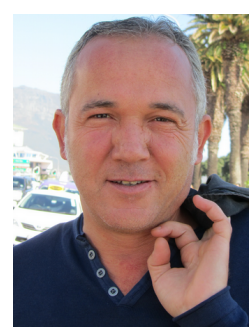

Tarek Hamel received the Ph.D. degree in robotics from University of Technology of Compiègne (UTC), Compiègne, France, in 1996.

After two years as a Research Assistant with UTC he joined Centre d'Etudes de Mécanique d'Ile de France in 1997 as an Associate Professor. He has been a Professor with University of Nice Sophia Antipolis, Nice, France, since 2003. His research interests include nonlinear control theory, estimation, and vision-based control with applications to unmanned aerial vehicles.

Prof. Hamel has served as an Associate Editor for IEEE Transactions on Robotics and Control Engineering Practice. 